\title{
ReSpect: Relativistic spectroscopy DFT program package
}

Cite as: J. Chem. Phys. 152, 184101 (2020); https://doi.org/10.1063/5.0005094

Submitted: 18 February 2020 . Accepted: 12 April 2020 . Published Online: 11 May 2020

Michal Repisky (D), Stanislav Komorovsky (D), Marius Kadek (D), Lukas Konecny (D), Ulf Ekström, Elena Malkin (D), Martin Kaupp (D), Kenneth Ruud (D), Olga L. Malkina (D), and Vladimir C. Malkin (D)

\section{COLLECTIONS}

Paper published as part of the special topic on Electronic Structure Software Note: This article is part of the JCP Special Topic on Electronic Structure Software.

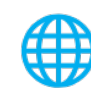

\section{Lock-in Amplifiers up to $600 \mathrm{MHz}$}

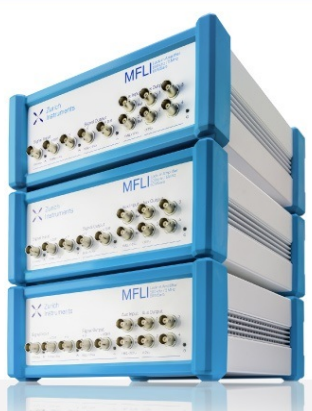




\title{
ReSpect: Relativistic spectroscopy DFT program package
}

\author{
Cite as: J. Chem. Phys. 152, 184101 (2020); doi: 10.1063/5.0005094 \\ Submitted: 18 February 2020 - Accepted: 12 April 2020 • \\ Published Online: 11 May 2020
}

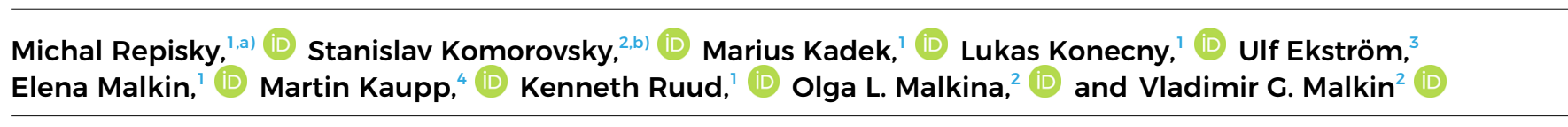

\section{AFFILIATIONS}

\author{
${ }^{1}$ Hylleraas Centre for Quantum Molecular Sciences, Department of Chemistry, UiT The Arctic University of Norway, \\ N-9037 Troms $\varnothing$, Norway \\ ${ }^{2}$ Institute of Inorganic Chemistry, Slovak Academy of Sciences, Dubravska cesta 9, SK-84536 Bratislava, Slovakia \\ ${ }^{3}$ Hylleraas Centre for Quantum Molecular Sciences, Department of Chemistry, University of Oslo, N-0315 Oslo, Norway \\ “Technische Universität Berlin, Institute of Chemistry, Strasse des 17 Juni 135, D-10623 Berlin, Germany
}

\begin{abstract}
Note: This article is part of the JCP Special Topic on Electronic Structure Software.
a) Author to whom correspondence should be addressed: michal.repisky@uit.no

${ }^{b}$ Electronic mail: stanislav.komorovsky@savba.sk
\end{abstract}

\begin{abstract}
With the increasing interest in compounds containing heavier elements, the experimental and theoretical community requires computationally efficient approaches capable of simultaneous non-perturbative treatment of relativistic, spin-polarization, and electron correlation effects. The ReSpect program has been designed with this goal in mind and developed to perform relativistic density functional theory (DFT) calculations on molecules and solids at the quasirelativistic two-component (X2C Hamiltonian) and fully relativistic four-component (Dirac-Coulomb Hamiltonian) level of theory, including the effects of spin polarization in open-shell systems at the Kramers-unrestricted self-consistent field level. Through efficient algorithms exploiting time-reversal symmetry, biquaternion algebra, and the locality of atom-centered Gaussian-type orbitals, a significant reduction of the methodological complexity and computational cost has been achieved. This article summarizes the essential theoretical and technical advances made in the program, supplemented by example calculations. ReSpect allows molecules with $>100$ atoms to be efficiently handled at the four-component level of theory on standard central processing unit-based commodity clusters, at computational costs that rarely exceed a factor of 10 when compared to the non-relativistic realm. In addition to the prediction of band structures in solids, ReSpect offers a growing list of molecular spectroscopic parameters that range from electron paramagnetic resonance parameters (g-tensor, A-tensor, and zero-field splitting), via (p)NMR chemical shifts and nuclear spin-spin couplings, to various linear response properties using either conventional or damped-response time-dependent DFT (TDDFT): excitation energies, frequency-dependent polarizabilities, and natural chiroptical properties (electronic circular dichroism and optical rotatory dispersion). In addition, relativistic real-time TDDFT electron dynamics is another unique feature of the program. Documentation, including user manuals and tutorials, is available at the program's website http://www.respectprogram.org.
\end{abstract}

Published under license by AIP Publishing. https://doi.org/10.1063/5.0005094

\section{INTRODUCTION}

The electronic motion in atoms, molecules, and solids is described by the (time-dependent) Dirac equation. ${ }^{1,2}$ However, the majority of quantum-chemical calculations use instead the (timedependent) Schrödinger equation, ${ }^{3}$ effectively assuming the speed of light to be infinite. The difference between the results obtained from solving the Dirac and the Schrödinger equations is often referred to as relativistic effects. These effects are, in general, assumed to be so small that they can be either ignored or treated approximately by using corrections to the Schrödinger equation. Such approximations either utilize effective core potentials ${ }^{4,5}$ or introduce 
correction operators derived from the Dirac-Coulomb-Breit Hamiltonian, ${ }^{6}$ which are applied either variationally or through perturbative corrections.

While relativistic effects can, in many cases, be safely ignored, there exist numerous examples where these effects are of tremendous importance even when considering the properties of light elements. When studying the core levels of atoms, as done in x-ray spectroscopy, ${ }^{8}$ it is well known that the three- and fivefold degeneracy of the orbital part of the atomic $p$ and $d$ functions is lifted by spin-orbit (SO) effects, giving rise to the $\mathrm{L}_{2,3} / \mathrm{M}_{2,3}$ and $\mathrm{M}_{4,5}$ edges, respectively. Consideration of relativistic spin-orbit effects is also essential when analyzing the electronic absorption spectra of compounds for medical imaging or photosensitizers involving lanthanides. 'Another example where relativistic effects are crucial for the analysis of spectroscopic observations is the so-called heavyatom on light-atom (HALA) effects observed in Nuclear Magnetic Resonance (NMR) spectroscopy, ${ }^{10,11}$ where relativistic corrections have been shown to far exceed the non-relativistic value even for the proton NMR shielding constant. ${ }^{12}$ Relativistic spin-orbit effects are also omnipresent in the solid state and at the core of important research areas in the development of novel materials for use in spintronics $^{13,14}$ and topological insulators. ${ }^{15,16}$ Relativistic effects have also been shown to affect reaction pathways in both inorganic ${ }^{17}$ and biochemical reactions. ${ }^{18}$

The fact that relativistic effects are ubiquitous and cannot a priori be ignored, combined with increased interest in heavyelement compounds that cannot be handled by more approximate methods, calls for computationally efficient treatments of the electronic structure of molecules and solids at the fully relativistic level of theory. The ReSpect program has been designed and developed with this challenge in mind, and this article provides an in-depth introduction to the program and, particularly, to its theoretical and algorithmic foundation that emerges as solutions to challenges posed by the two- and four-component relativistic theory. In addition, the program's capabilities, in terms of both functionality and performance, are demonstrated for applications where the variational treatment of relativistic corrections, in particular the spin-orbit interaction, is indispensable.

Work on the ReSpect code started around 1998 by two of the co-authors of this paper (V.M. and O.M.). Before 1998, they had been working with the DEMON code ${ }^{19}$ developing original methods for DFT calculations of NMR and electron paramagnetic resonance (EPR) parameters, including spin-orbit corrections to NMR shifts. ${ }^{20-24}$ Further method development within the DEMON code became problematic, however, due to the absence of four-center integrals, among other limitations of the program. Moreover, the publication of pioneering papers on the calculation of the EPR gtensor by two-component (2c) approaches ${ }^{25,26}$ stimulated the decision to write a new and flexible $2 c$ code specifically designed for relativistic DFT and Hartree-Fock calculations of NMR and EPR parameters. The primary goal was to create a "sandbox" for exploring new ideas and developing new methods, computational efficiency being at the time a secondary issue. This code was named ReSpect. This early version of the ReSpect code was made possible by the very kind permission by Dennis Salahub for the use of parts of the DEMON code and from the authors of the HERMIT integral block. ${ }^{27}$ At the beginning of the development of the $2 \mathrm{c}$ ReSpect code, great help was provided by the late Bernd Schimmelpfennig and also by Roman Reviakine. This original code allowed a few groups to easily implement and explore new ideas in the field of $2 c$ relativistic methods. A new era of the code started when M.R. and S.K. joined the Malkin group. The original program was fully re-designed, re-implemented, and significantly extended in order to make it an efficient two- and four-component DFT program, including a new integral block InteRest developed by M.R. and tailored specifically for relativistic calculations. Due to the philosophy and structure of the new ReSpect, the program inherited the old code's raison d'etre of providing a platform for exploring new ideas, but with the addition of high computational efficiency and parallel scalability for production calculations. The most recent era of the program started around 2010 when K.R. brought to the project his expertise with calculations of dynamical molecular properties. Since then, the program has grown beyond the domain of magnetic properties and now includes some fastdeveloping theoretical and computational areas such as real-time electron dynamics and a relativistic electronic structure of periodic systems, pursued mainly by the younger team members (L.K. and M.K.).

Currently, ReSpect supports a variety of exchange-correlation (XC) functionals and basis sets suitable for relativistic property calculations. Basis sets are of uncontracted form and built with primitive Gaussian-type functions. The standard sets in the ReSpect database range in quality from minimal double-zeta to very large quadruple-zeta plus polarization. To maximize performance, various advanced computational techniques have been implemented in ReSpect, such as a vectorized and parallel integral library InteRest, an efficient and parallel XC numerical integrator, RI-J technology for the two-electron Coulomb problem, and hybrid Message Passing Interface (MPI)/OpenMP parallelization.

ReSpect is distributed as proprietary software at no cost and is currently available as OpenMP parallel static binaries for Linux OS and x86_64 architecture on the program's home website: http://www.respectprogram.org. The program is limited to noncommercial, academic, and non-profit private use. More specific license terms, in addition to the documentation, user manuals, and tutorials, are available on the program's homepage. A comprehensive list of functionalities that are either part of the current stable release or are in the performance optimization phase (the beta version) is given in Table I. Estimates for the dates of forthcoming public releases of novel features are provided on the ReSpect homepage.

The rest of this paper is organized as follows: in Sec. II, we provide some technical information about the underlying theoretical basis on which the functionality and efficiency of the ReSpect program is based. The reader more interested in the chemistry that can be studied with the program may choose to skip this section. In Sec. III, we outline how the molecular Kohn-Sham determinants and densities are optimized and give some illustrative examples of the systems that can be addressed by ReSpect. Section IV provides a similar overview for the solid-state functionality of ReSpect. Section $V$ gives an overview of the functionality for calculating electron paramagnetic resonance (EPR) parameters, and Secs. VI and VII provide an overview of the calculation of nuclear magnetic shielding (NMR) properties for closed-shell and open-shell molecules, respectively. Sections VIII-X describe how dynamic, 
TABLE I. List of properties implemented in ReSpect alongside the specification of Hamiltonians, theoretical methods, Kramers-restricted/unrestricted formalisms, and literature references. Abbreviations in alphabetical order: DR, damped response TDDFT; EAS, electronic absorption spectroscopy; ECD, electronic circular dichroism; $E E$, excitation energies; $E F G$, electric field gradient; $K R$, Kramers-restricted; $K U$, Kramers-unrestricted; LR, linear response TDDFT; NSR, nuclear spin-rotation constant; ORD, optical rotatory dispersion; PCM, polarizable continuum model; PT1/2, static perturbation theory of the first/second order; RT, real-time TDDFT; and ZFS, zero-field splitting. The boldface values refer to a different spectroscopy classes.

\begin{tabular}{lllll}
\hline \hline Property & Hamiltonian & Method KR KU & Reference
\end{tabular}

Self-consistent field (SCF)

\begin{tabular}{lccc}
\hline Molecular & $1 \mathrm{c}, 2 \mathrm{c}, 4 \mathrm{c}$ & $\checkmark$ & $\checkmark$ \\
Solid-state & $1 \mathrm{c}, 4 \mathrm{c}$ & $\checkmark$ &
\end{tabular}

Electron paramagnetic resonance (EPR)

\begin{tabular}{llllll}
\hline$g$-tensor & $4 \mathrm{c}$ & PT1 & - & $\checkmark$ & 29 and 30 \\
$A$-tensor & $4 \mathrm{c}$ & PT1 & - & $\checkmark$ & 31 and 30 \\
ZFS & $4 \mathrm{c}$ & LR & - & $\checkmark$ & This work
\end{tabular}

Nuclear magnetic resonance (NMR)

\begin{tabular}{lllllc}
\hline$\sigma$-tensor & $4 \mathrm{c}$ & PT2 & $\checkmark$ & - & 32 and 33 \\
$J$-tensor & $4 \mathrm{c}$ & PT2 & $\checkmark$ & - & 34
\end{tabular}

Paramagnetic nuclear magnetic resonance (pNMR)

\begin{tabular}{llcccc}
\hline$\sigma$-tensor & $4 \mathrm{c}$ & PT2 & - & $\checkmark$ & 35 and 36 \\
$J$-tensor & $4 \mathrm{c}$ & PT2 & - & $\checkmark$ & This work \\
\multicolumn{5}{c}{ Optical properties } \\
\end{tabular}

\begin{tabular}{lccccc}
\hline EE/EAS & $1 \mathrm{c}, 2 \mathrm{c}, 4 \mathrm{c}$ & RT & $\checkmark$ & $\checkmark$ & 37 \\
& $1 \mathrm{c}, 2 \mathrm{c}, 4 \mathrm{c}$ & DR & $\checkmark$ & & 38 \\
Polarizability & $1 \mathrm{c}, 4 \mathrm{c}$ & LR & $\checkmark$ & $\checkmark$ & 39, this work \\
& $1 \mathrm{c}, 2 \mathrm{c}, 4 \mathrm{c}$ & RT & $\checkmark$ & $\checkmark$ & 40 \\
& $1 \mathrm{c}, 2 \mathrm{c}, 4 \mathrm{c}$ & DR & $\checkmark$ & & 38 \\
X-ray EAS & $1 \mathrm{c}, 4 \mathrm{c}$ & LR & $\checkmark$ & $\checkmark$ & 39 , this work \\
& 1c, 2c, 4c & RT & $\checkmark$ & $\checkmark$ & 41 \\
Rad. lifetimes & 1c, 2c, 4c & DR & $\checkmark$ & & This work \\
& $1 \mathrm{c}, 4 \mathrm{c}$ & LR & $\checkmark$ & $\checkmark$ & 39, this work \\
& \multicolumn{7}{c}{ Natural chiroptical properties } & \\
\hline
\end{tabular}

\begin{tabular}{llllll}
\hline ECD & 1c, 2c, 4c & RT & $\checkmark$ & $\checkmark$ & 42 \\
& $1 \mathrm{c}, 2 \mathrm{c}, 4 \mathrm{c}$ & DR & $\checkmark$ & & 38 \\
ORD & 1c, 2c, 4c & RT & $\checkmark$ & $\checkmark$ & 42 \\
& $1 \mathrm{c}, 2 \mathrm{c}, 4 \mathrm{c}$ & DR & $\checkmark$ & & 38 \\
& & Additional properties & &
\end{tabular}

\begin{tabular}{|c|c|c|c|c|}
\hline EFG & $1 c, 2 c, 4 c$ & PT1 & $\checkmark$ & \\
\hline Mossbauer & $1 c, 2 c, 4 c$ & PT1 & $\checkmark$ & \\
\hline NSR & $1 c, 4 c$ & PT2 & $\checkmark$ & 43 and 44 \\
\hline \multicolumn{5}{|c|}{ Continuum solvent model } \\
\hline PCM & $1 c, 2 c, 4 c$ & & $\checkmark$ & 45 and 46 \\
\hline
\end{tabular}

linear-response properties can be obtained using the ReSpect implementation of real-time, damped response of linear response timedependent density functional theory. Finally, some concluding remarks are given in Sec. XI.

\section{GENERAL}

The relativistic description of many-electron systems poses several theoretical and technical challenges, in particular, those associated with the choice, design, and algebraic manipulation of basis sets suitable for relativistic calculations involving electric and magnetic fields. The necessity of using kinetically/magnetically balanced basis introduces additional theoretical and algorithmic challenges associated with the efficient evaluation and processing of electron repulsion integrals. In addition, to fully capture spin-orbit coupling effects and to ensure rotational invariance of the total energy, the standard non-relativistic collinear parameterization of DFT functionals is no longer adequate. To effectively deal with the increased complexity of relativistic theory, several advances have been made in the ReSpect program. The main goal of this section is to highlight these fundamental theoretical and technical concepts.

As discussed throughout this work, the unique ability of the ReSpect program is to treat both closed- and open-shell systems at the relativistic level. However, in the relativistic DFT framework with spin-orbit included variationally, it is somewhat unfortunate to refer to a Kohn-Sham (KS) determinant as a closed-shell or an open-shell. In this work, therefore, we will use "closed-shell KS determinant" to mean a single-determinant Kramers-restricted wave function and "open-shell KS determinant" to mean a singledeterminant Kramers-unrestricted wave function. In this terminology, closed-shell systems have a non-degenerate ground state with a time-reversal symmetric density matrix, and open-shell systems have a degenerate ground state with a density matrix without any time-reversal symmetry. For the sake of simplicity of language, throughout this paper, we use "Kohn-Sham determinant" to mean the determinant of a reference system of non-interacting electrons.

Unless otherwise stated, the Hartree system of atomic units will be employed, setting the elementary charge, the electron rest mass, and reduced Planck's constant to unity. Throughout this paper, Einstein's implicit summation over repeated indices is assumed.

\section{A. Dirac-Coulomb and $\mathrm{X} 2 \mathrm{C}$ Hamiltonians}

The ReSpect program utilizes the four-component (4c) manyparticle Dirac-Coulomb (DC) Hamiltonian, composed of a sum of the single-particle Dirac Hamiltonian $\hat{h}^{\mathrm{D}}$ and the two-particle interaction Hamiltonian derived from the non-relativistic instantaneous Coulomb interaction $\hat{g}\left(\boldsymbol{r}_{1}, \boldsymbol{r}_{2}\right)=1 /\left|\boldsymbol{r}_{1}-\boldsymbol{r}_{2}\right|$ between $N$ particles,

$$
\hat{H}=\sum_{i}^{N} \hat{h}_{i}^{\mathrm{D}}+\frac{1}{2} \sum_{i \neq j}^{N} \hat{g}\left(\boldsymbol{r}_{i}, \boldsymbol{r}_{j}\right) .
$$

Within the Born-Oppenheimer approximation, $\hat{h}^{\mathrm{D}}$ describes a relativistic spin-1/2 particle with charge $q$ moving in $\mathbb{R}^{3}$ and interacting with an external electrostatic scalar potential $\phi(\boldsymbol{r})$ due to the fixed atomic nuclei, ${ }^{1,2}$

$$
\begin{aligned}
\hat{h}^{\mathrm{D}} & =\left(\beta-\mathrm{I}_{4}\right) c^{2}+c(\boldsymbol{\alpha} \cdot \boldsymbol{p})+q \phi(\boldsymbol{r}) \mathrm{I}_{4} \\
& =\left(\begin{array}{cc}
q \phi(\boldsymbol{r}) \mathrm{I}_{2} & c(\boldsymbol{\sigma} \cdot \boldsymbol{p}) \\
c(\boldsymbol{\sigma} \cdot \boldsymbol{p}) & \left(q \phi(\boldsymbol{r})-2 c^{2}\right) \mathrm{I}_{2}
\end{array}\right) .
\end{aligned}
$$


Here, $\mathrm{I}_{n}$ is the $n \times n$ identity matrix, $c \sim 137$ is the speed of light, $\boldsymbol{r} \in \mathbb{R}^{3}$ is the particle position vector, and $\boldsymbol{p}=-i\left(\partial_{x}, \partial_{y}, \partial_{z}\right)$ is the canonical momentum operator. In 1928, Dirac introduced four new dynamical variables,

$$
\beta:=\left(\begin{array}{cc}
\mathrm{I}_{2} & 0_{2} \\
0_{2} & -\mathrm{I}_{2}
\end{array}\right), \quad \boldsymbol{\alpha}:=\left(\begin{array}{cc}
0_{2} & \boldsymbol{\sigma} \\
\boldsymbol{\sigma} & 0_{2}
\end{array}\right),
$$

in order to formulate a relativistic quantum-mechanical equation of motion for an electron subjected to an electromagnetic field. These new variables, the so-called Dirac matrices, fulfill the anticommutation rules

$$
\left[\alpha_{k}, \beta\right]_{+}=0, \quad\left[\alpha_{k}, \alpha_{l}\right]_{+}=2 \delta_{k l} \mathrm{I}_{4}
$$

and are customarily constructed using the two-component (2c) Pauli spin matrices

$$
\sigma_{1}:=\left(\begin{array}{ll}
0 & 1 \\
1 & 0
\end{array}\right), \quad \sigma_{2}:=\left(\begin{array}{cc}
0 & -\mathrm{i} \\
\mathrm{i} & 0
\end{array}\right), \quad \sigma_{3}:=\left(\begin{array}{cc}
1 & 0 \\
0 & -1
\end{array}\right) .
$$

The properties and the physical interpretation of the Dirac Hamiltonian can be found in several standard textbooks ${ }^{6,7,47,48}$ and will therefore not be covered extensively here. Instead, we will summarize some important points that are of relevance for the rest of this paper.

\section{Energy spectrum of $\hat{\mathrm{h}}^{\mathrm{D}}$}

In the absence of an external potential $\phi(r)$ in the Dirac Hamiltonian, the solution of the time-independent Dirac wave equation,

$$
\left[\beta c^{2}+c(\boldsymbol{\alpha} \cdot \boldsymbol{p})\right] \psi=E \psi,
$$

gives a continuous spectrum of scattering states lying in two disjoint energy intervals $\left(-\infty,-c^{2}\right\rangle$ and $\left\langle c^{2}, \infty\right)$. In contrast, the presence of an attractive interaction potential in Eq. (6), such that the potential energy of interaction of the electron $(q=-1)$ with the external field $\langle V\rangle:=\langle q \phi\rangle$ is within $0>\langle V\rangle>-2 c^{2}$, gives rise to a countable set of discrete electronic bound states in the energy region $c^{2}>E>-c^{2} .{ }^{49}$ These bound eigenstates are of importance for relativistic quantum chemistry and bear the form of four-component functions in the complex linear space $\mathbb{C}^{4}$-the Dirac spinors

$$
\psi(\boldsymbol{r})=\left(\begin{array}{c}
\psi^{\mathrm{L}} \\
\psi^{S}
\end{array}\right)=\left(\begin{array}{l}
\psi^{1} \\
\psi^{2} \\
\psi^{3} \\
\psi^{4}
\end{array}\right) \in \mathbb{C}^{4}\left(\mathbb{R}^{3}\right) .
$$

The notation in terms of the $2 c$ large-component $\left(\psi^{\mathrm{L}}\right)$ and smallcomponent $\left(\psi^{\mathrm{S}}\right)$ Pauli spinors reflects the relative size of these components $-\psi^{\mathrm{L}}$ is of the order $\mathcal{O}\left(c^{0}\right)$ and gives the dominant contribution to the positive-energy solutions, whereas $\psi^{S}$ is a factor $c$ smaller (and vice versa for the negative-energy solutions).

\section{Nuclear model}

In non-relativistic theory, the external electrostatic scalar potential $\phi(\boldsymbol{r})$ is often given in terms of a point-charge nuclear model (pn),

$$
\phi^{\mathrm{pn}}(\boldsymbol{r})=\sum_{A}^{M} Z_{A} \phi_{A}^{\mathrm{pn}}(\boldsymbol{r})=\sum_{A}^{M} \frac{Z_{A}}{\left|\boldsymbol{r}-\boldsymbol{R}_{\boldsymbol{A}}\right|},
$$

where the summation runs over $M$ fixed nuclei, each characterized by a nuclear charge $Z$ and a position vector $\boldsymbol{R}$. An advantage of this model is that it has the same form for all nuclei, independent of their nuclear mass. However, the use of a point-charge nuclear model in relativistic calculations results in a weak singularity in the electronic wave function at the nucleus (the wave function remains normalizable), and not a cusp as in the non-relativistic case. Since the weak singularity cannot be described by the conventional finite basis sets of Slater or Gaussian type, the point-charge model is replaced by a finite-sized nuclear model (fn) that results in a regularized wave function at the origin. For integral efficiency reasons, ReSpect adopts the finite-size nuclear model of Gaussian type, with a parameterization according to Ref. 50,

$$
\phi^{\mathrm{fn}}(\boldsymbol{r})=\sum_{A}^{M} Z_{A} \phi_{A}^{\mathrm{fn}}(\boldsymbol{r})=\sum_{A}^{M} Z_{A} \int \frac{G_{A}\left(\left|\boldsymbol{R}-\boldsymbol{R}_{A}\right|\right)}{|\boldsymbol{r}-\boldsymbol{R}|} d^{3} \boldsymbol{R} .
$$

Here, $G_{A}$ refers to a normalized s-type Gaussian function

$$
G_{A}=\left(\frac{\eta_{A}}{\pi}\right)^{3 / 2} \exp \left[-\eta_{A}\left(\boldsymbol{R}-\boldsymbol{R}_{A}\right)^{2}\right]
$$

with an element-specific exponent $\eta_{A}$ related to the nuclear mass of the element.

\section{Exact two-component (X2C) Hamiltonian}

The higher computational cost traditionally associated with solving the $4 \mathrm{c}$ Dirac equation has motivated the development of the computationally less demanding quasirelativistic $2 c$ Hamiltonians. Of these, $\mathrm{X} 2 \mathrm{C}$ has gained popularity in recent years because it reduces the original $4 \mathrm{c}$ problem to its $2 \mathrm{c}$ form at the expense of applying only simple algebraic manipulations, without the need for generating explicit operator expressions for higher-order relativistic corrections and/or property operators. ${ }^{51-53}$ While the computational cost of any $2 \mathrm{c}$ approach with full picture-change-transformed one- and two-electron integrals is even higher than the parent $4 \mathrm{c}$ problem, the main efficiency gain comes from the additional approximation of neglecting this picture change transformation for the two-electron integrals. This approximation is also adopted in ReSpect. The current implementation of X2C utilizes the one-step noniterative $\mathrm{X} 2 \mathrm{C}$ decoupling procedure of Ilias and Saue ${ }^{53}$ and facilitates block diagonalization of the one-electron Dirac Hamiltonian represented in a restricted kinetically balanced (RKB) spinor basis $\mathbf{h}^{\mathrm{D}}$,

$$
\mathbf{h}^{\mathrm{D}} \rightarrow \mathbf{h}^{\mathrm{X} 4 \mathrm{C}} \equiv \mathbf{U}^{\dagger} \mathbf{h}^{\mathrm{D}} \mathbf{U}=\left(\begin{array}{cc}
\mathbf{h}_{+}^{\mathrm{X} 4 \mathrm{C}} & 0 \\
0 & \mathbf{h}_{-}^{\mathrm{X} 4 \mathrm{C}}
\end{array}\right) \rightarrow \mathbf{h}^{\mathrm{X} 2 \mathrm{C}} \equiv \mathbf{h}_{+}^{\mathrm{X} 4 \mathrm{C}} .
$$

The unitary decoupling matrix $\mathbf{U}$ is obtained from the eigenvectors of $\mathbf{h}^{\mathrm{D}}$ by solving a simple set of algebraic equations. More details about the X2C implementation in ReSpect are provided in Ref. 40.

\section{B. Restricted kinetically balanced (RKB) basis}

The search for single-particle eigenfunctions [Eq. (7)] of the Dirac Hamiltonian is performed in ReSpect in the space spanned 
by $4 n$ basis spinors $\left\{\chi_{a}^{1}(\boldsymbol{r}), \ldots, \chi_{a}^{4}(\boldsymbol{r})\right\}_{a=1}^{n}$,

$$
\psi(\boldsymbol{r})=\sum_{a=1}^{n} C_{a}^{1} \chi_{a}^{1}(\boldsymbol{r})+C_{a}^{2} \chi_{a}^{2}(\boldsymbol{r})+C_{a}^{3} \chi_{a}^{3}(\boldsymbol{r})+C_{a}^{4} \chi_{a}^{4}(\boldsymbol{r})
$$

where $n$ refers to the size of a scalar basis and $C_{a}^{1-4} \in \mathbb{C}$ are distinct scalar expansion coefficients defined in the field of complex numbers. The four different terms in Eq. (12) reflect the spin and charge degrees of freedom associated with the particle in question. If $\psi(\boldsymbol{r})$ denotes an electronic solution of the Dirac equation, then the large-component basis spinors $\chi_{a}^{1}(\boldsymbol{r})$ and $\chi_{a}^{2}(\boldsymbol{r})$ can, just as in the non-relativistic realm, be constructed as a direct product of a scalar real-valued spatial function $f_{a}(\boldsymbol{r}) \in \mathbb{R}\left(\mathbb{R}^{3}\right)$ and a spin vector in $\mathbb{R}^{4}$,

$$
\begin{aligned}
& \chi_{a}^{1}(\boldsymbol{r}):=\mathcal{N}_{a}^{\mathrm{L}}\left(\begin{array}{l}
1 \\
0 \\
0 \\
0
\end{array}\right) f_{a}(\boldsymbol{r}) \in \mathbb{R}^{4}\left(\mathbb{R}^{3}\right), \\
& \chi_{a}^{2}(\boldsymbol{r}):=\mathcal{N}_{a}^{\mathrm{L}}\left(\begin{array}{l}
0 \\
1 \\
0 \\
0
\end{array}\right) f_{a}(\boldsymbol{r}) \in \mathbb{R}^{4}\left(\mathbb{R}^{3}\right) .
\end{aligned}
$$

In this notation, we select the eigenvectors of the Pauli spin $z$ matrix as the spin basis and $f_{a}(\boldsymbol{r})$ as the spatial basis. In ReSpect, $f_{a}(\boldsymbol{r})$ is chosen to be an ordinary non-relativistic scalar basis function of the Gaussian type. The normalization factor $\mathcal{N}_{a}^{\mathrm{L}} \in \mathbb{R}>0$ is obtained from the orthonormality condition,

$$
\int_{\mathbb{R}^{3}}\left(\chi_{a}^{k}(\boldsymbol{r})\right)^{\dagger} \chi_{b}^{l}(\boldsymbol{r}) d^{3} \boldsymbol{r}=\delta_{a b} \delta_{k l} ; \quad k, l \in 1-4,
$$

where $\left(\chi_{a}^{k}\right)^{\dagger}$ denotes complex conjugation and transposition of $\chi_{a}^{k}$. For $k \in 1,2$ and non-zero $f_{a}(\boldsymbol{r})$, the normalization condition reduces to the evaluation of the reciprocal square root of the overlap integrals.

The two remaining spinors $\chi_{a}^{3}(\boldsymbol{r})$ and $\chi_{a}^{4}(\boldsymbol{r})$ in Eq. (12) form the basis for the small-component $\psi^{S}$ in Eq. (7), and their contribution dominates in the solutions associated with a charge-conjugated particle (positron). Early numerical experiments with the variational solution of the Dirac equation in a strictly identical spinor basis for both $\psi^{\mathrm{L}}$ and $\psi^{\mathrm{S}}$ resulted in severe shortcomings in the computed kinetic energy that persisted even in the nonrelativistic limit. ${ }^{54}$ Schwarz and Wallmeier related this problem to an inadequate basis representation of $\psi^{\mathrm{S}}$ to which they attributed the primary mechanism of variational failures observed in the early days of relativistic molecular calculations. ${ }^{55}$ As shown by Stanton and Havriliak, the error in the kinetic energy can be significantly reduced if the Dirac eigenstates are expanded directly in a finite basis satisfying the so-called restricted kinetically balanced (RKB) relation. ${ }^{56} \mathrm{RKB}$ thus relates $\chi_{a}^{3}(\boldsymbol{r})$ and $\chi_{a}^{4}(\boldsymbol{r})$ to their large-component basis counterparts defined by Eq. (13). In the absence of any electromagnetic vector potential in the Dirac Hamiltonian, the RKB relation takes the following operator form: ${ }^{56}$

$$
\begin{aligned}
\hat{\mathcal{O}}^{\mathrm{RKB}} & :=\frac{1}{2 c} \boldsymbol{\alpha} \cdot \boldsymbol{p} \\
& =\frac{-i}{2 c}\left(\begin{array}{cccc}
0 & 0 & \partial_{z} & \partial_{x}-i \partial_{y} \\
0 & 0 & \partial_{x}+i \partial_{y} & -\partial_{z} \\
\partial_{z} & \partial_{x}-i \partial_{y} & 0 & 0 \\
\partial_{x}+i \partial_{y} & -\partial_{z} & 0 & 0
\end{array}\right) .
\end{aligned}
$$

Applying the RKB operator to the large-component basis spinors in Eq. (13), one obtains the analytic expression for the basis spinors of the small component,

$$
\begin{aligned}
\chi_{a}^{3}(\boldsymbol{r}) & =\mathcal{N}_{a} \hat{\mathcal{O}}^{\mathrm{RKB}} \chi_{a}^{1}(\boldsymbol{r}) \\
& =\mathcal{N}_{a}^{S}\left(\begin{array}{c}
0 \\
0 \\
-i \partial_{z} \\
\partial_{y}-i \partial_{x}
\end{array}\right) f_{a}(\boldsymbol{r}) \in \mathbb{C}^{4}\left(\mathbb{R}^{3}\right), \\
\chi_{a}^{4}(\boldsymbol{r}) & =\mathcal{N}_{a} \hat{\mathcal{O}}^{\mathrm{RKB}} \chi_{a}^{2}(\boldsymbol{r}) \\
& =\mathcal{N}_{a}^{S}\left(\begin{array}{c}
0 \\
0 \\
-\partial_{y}-i \partial_{x} \\
i \partial_{z}
\end{array}\right) f_{a}(\boldsymbol{r}) \in \mathbb{C}^{4}\left(\mathbb{R}^{3}\right),
\end{aligned}
$$

written in terms of Cartesian derivatives of the scalar basis $f_{a}(\boldsymbol{r})$. Here, $\mathcal{N}_{a}^{\mathrm{S}} \equiv \mathcal{N}_{a} \mathcal{N}_{a}^{\mathrm{L}} /(2 c)$ is the small-component normalization factor derived from Eq. (14) whose evaluation requires the reciprocal square root of the scaled kinetic energy integrals,

$$
\mathcal{N}_{a}^{S}=\left[\int_{\mathbb{R}^{3}}\left(\boldsymbol{\partial} f_{a}(\boldsymbol{r})\right) \cdot\left(\boldsymbol{\partial} f_{a}(\boldsymbol{r})\right) d^{3} \boldsymbol{r}\right]^{-1 / 2} \in \mathbb{R}>0 .
$$

A very important consequence of the preceding discussion is the fact that the expansion in Eq. (12), together with the explicit form for $\chi_{a}^{1}-\chi_{a}^{4}$ derived from $\mathrm{RKB}$, can be recast in a compact matrix form [with the spinor components $\psi^{2}$ and $\psi^{3}$ swapped as compared to Eq. (7)],

$$
\begin{aligned}
\psi(\boldsymbol{r}) & =\sum_{a=1}^{n} X_{a}^{\mathrm{RKB}}(\boldsymbol{r}) C_{a} \\
& =\sum_{a=1}^{n}\left(\begin{array}{cccc}
f_{a}^{\mathrm{L}} & 0 & 0 & 0 \\
0 & -i \partial_{z} f_{a}^{\mathrm{S}} & 0 & -\left(\partial_{y}+i \partial_{x}\right) f_{a}^{\mathrm{S}} \\
0 & 0 & f_{a}^{\mathrm{L}} & 0 \\
0 & \left(\partial_{y}-i \partial_{x}\right) f_{a}^{\mathrm{S}} & 0 & i \partial_{z} f_{a}^{\mathrm{S}}
\end{array}\right)\left(\begin{array}{c}
C_{a}^{1} \\
C_{a}^{3} \\
C_{a}^{2} \\
C_{a}^{4}
\end{array}\right) .
\end{aligned}
$$

Here, a short-hand notation for elemental scalar functions $f_{a}^{\mathrm{L}} \equiv \mathcal{N}_{a}^{\mathrm{L}} f_{a}(\boldsymbol{r})$ and $f_{a}^{\mathrm{S}} \equiv \mathcal{N}_{a}^{\mathrm{S}} f_{a}(\boldsymbol{r})$ has been introduced. The matrix notation for the basis in Eq. (18) provides not only a direct link between the relativistic basis $\boldsymbol{X}^{\mathrm{RKB}}(\boldsymbol{r}) \in \mathbb{C}^{n \times 4 n}\left(\mathbb{R}^{3}\right)$ and its nonrelativistic scalar counterpart $\boldsymbol{f}(\boldsymbol{r}) \in \mathbb{R}^{n}\left(\mathbb{R}^{3}\right)$ but also a mathematical foundation for introducing the relativistic $\mathrm{RKB}$ basis as real quaternion functions $\mathbb{H}_{\mathbb{R}}^{2 \times 2}$ in $\mathbb{R}^{3}$. This quaternion-based formalism has 
significant computational benefits, as we shall discuss later in this section, which can be recognized by noting that every basis spinor in Eq. (18) has a built-in time-reversal symmetric (TRS) structure, i.e.,

$$
X_{a}^{\mathrm{RKB}} \equiv X_{a}^{\mathrm{RKB}}(\boldsymbol{r})=\left(\begin{array}{cc}
\mathcal{A} & \mathcal{B} \\
-\mathcal{B}^{*} & \mathcal{A}^{*}
\end{array}\right)_{a} \in \mathbb{C}_{+}^{4 \times 4}\left(\mathbb{R}^{3}\right),
$$

with elements

$$
\begin{aligned}
& \mathcal{A}_{a} \equiv \mathcal{A}_{a}(\boldsymbol{r}):=\left(\begin{array}{cc}
f^{\mathrm{L}}(\boldsymbol{r}) & 0 \\
0 & -i \partial_{z} f^{\mathrm{S}}(\boldsymbol{r})
\end{array}\right)_{a} \in \mathbb{C}^{2 \times 2}\left(\mathbb{R}^{3}\right), \\
& \mathcal{B}_{a} \equiv \mathcal{B}_{a}(\boldsymbol{r}):=\left(\begin{array}{cc}
0 & 0 \\
0 & -\left(\partial_{y}+i \partial_{x}\right) f^{\mathrm{S}}(\boldsymbol{r})
\end{array}\right)_{a} \in \mathbb{C}^{2 \times 2}\left(\mathbb{R}^{3}\right) .
\end{aligned}
$$

Furthermore, there exists an algebraic isomorphism between the algebra of $4 \times 4$ complex TRS matrices and the algebra of $2 \times 2$ quaternion matrices over the field of real numbers $\mathbb{R}$, i.e., $\mathbb{C}_{+}^{4 \times 4} \simeq \mathbb{H}_{\mathbb{R}}^{2 \times 2}$, such that for every $q \in \mathbb{C}_{+}^{4 \times 4}$, it holds that

$$
q=\left(\begin{array}{cc}
q_{0}+i q_{1} & q_{2}+i q_{3} \\
-q_{2}+i q_{3} & q_{0}-i q_{1}
\end{array}\right) \simeq q_{0}+q_{1} \mathbf{i}+q_{2} \mathbf{j}+q_{3} \mathbf{k} \in \mathbb{H}_{\mathbb{R}}^{2 \times 2} .
$$

Here, $q_{0}, q_{1}, q_{2}, q_{3}$ are $2 \times 2$ matrices over $\mathbb{R}$, and $\mathbf{i}, \mathbf{j}, \mathbf{k}$ are orthogonal unit vectors in $\mathbb{H}$, which satisfy the identities,

$$
\mathbf{i}^{2}=\mathbf{j}^{2}=\mathbf{k}^{2}=\mathbf{i j k}=-1,
$$

and are represented in $\mathbb{C}^{2 \times 2}$ by the product of the Pauli spin matrices $\sigma_{1}, \sigma_{2}, \sigma_{3}$ with the imaginary unit $i$,

$$
\mathbf{i} \simeq\left(\begin{array}{cc}
i & 0 \\
0 & -i
\end{array}\right), \quad \mathbf{j} \simeq\left(\begin{array}{cc}
0 & 1 \\
-1 & 0
\end{array}\right), \quad \mathbf{k} \simeq\left(\begin{array}{ll}
0 & i \\
i & 0
\end{array}\right) .
$$

An essential consequence of this isomorphism is that it is possible to perform an isomorphic mapping of the relativistic TRS RKB spinor basis $X_{a}^{\mathrm{RKB}}(\boldsymbol{r})$ in Eqs. 19 and 20 into a real quaternion format, ${ }^{q} X_{a}^{\mathrm{RKB}}(\boldsymbol{r})$,

$$
X_{a}^{\mathrm{RKB}}(\boldsymbol{r}) \simeq{ }^{q} X_{a}^{\mathrm{RKB}}(\boldsymbol{r})={ }^{0} X_{a}+{ }^{1} X_{a} \mathbf{i}+{ }^{2} X_{a} \mathbf{j}+{ }^{3} X_{a} \mathbf{k} \in \mathbb{H}_{\mathbb{R}}^{2 \times 2}\left(\mathbb{R}^{3}\right),
$$

with the quaternion constituents defined as

$$
\begin{aligned}
& { }^{0} X_{a}(\boldsymbol{r})=\mathfrak{R}\left(\mathcal{A}_{a}\right)=\left(\begin{array}{cc}
f^{\mathrm{L}}(\boldsymbol{r}) & 0 \\
0 & 0
\end{array}\right) \in \mathbb{R}_{a}^{2 \times 2}\left(\mathbb{R}^{3}\right), \\
& { }^{1} X_{a}(\boldsymbol{r})=\mathfrak{I}\left(\mathcal{A}_{a}\right)=\left(\begin{array}{cc}
0 & 0 \\
0 & -\partial_{z} f^{\mathrm{S}}(\boldsymbol{r})
\end{array}\right)_{a} \in \mathbb{R}^{2 \times 2}\left(\mathbb{R}^{3}\right), \\
& { }^{2} X_{a}(\boldsymbol{r})=\mathfrak{R}\left(\mathcal{B}_{a}\right)=\left(\begin{array}{cc}
0 & 0 \\
0 & -\partial_{y} f^{\mathrm{S}}(\boldsymbol{r})
\end{array}\right)_{a} \in \mathbb{R}^{2 \times 2}\left(\mathbb{R}^{3}\right), \\
& { }^{3} X_{a}(\boldsymbol{r})=\Im\left(\mathcal{B}_{a}\right)=\left(\begin{array}{cc}
0 & 0 \\
0 & -\partial_{x} f^{\mathrm{S}}(\boldsymbol{r})
\end{array}\right) \in \mathbb{R}_{a}^{2 \times 2}\left(\mathbb{R}^{3}\right) .
\end{aligned}
$$

Note that the real quaternion format for the RKB basis can be used as a starting point for formulating $4 \mathrm{c}$ electronic structure theory in the algebra of quaternions. As discussed in Subsection I C, the quaternion formalism can also be applied to relativistic bases that satisfy the restricted magnetically balanced (RMB) condition. ${ }^{32}$ $\mathrm{RMB}$ plays an indispensable role in $4 \mathrm{c}$ calculations that involve an electromagnetic vector potential as a perturbation. ${ }^{32-34}$

Let us conclude this section by introducing a compact notation for quaternions that will be useful in Secs. II C, II D, and III. It utilizes the decomposition of ${ }^{q} X_{a}$ into a sum of a scalar and a vector, called the real part $\mathcal{R}\left({ }^{q} X_{a}\right)={ }^{0} X_{a}$ and the pure part $\mathcal{P}\left({ }^{q} X_{a}\right)$ $={ }^{1} X_{a} \mathbf{i}+{ }^{2} X_{a} \mathbf{j}+{ }^{3} X_{a} \mathbf{k}$. It is convenient to denote the pure part by a boldface letter so that ${ }^{q} X_{a}={ }^{0} X_{a}+X_{a}$.

\section{Restricted magnetically balanced (RMB) basis}

The coupling of a quantum molecular system to a classical static electromagnetic field derived from the time-independent scalar potential $\phi(\boldsymbol{r})$ and vector potential $\boldsymbol{A}(\boldsymbol{r})$ is performed through the principle of minimal electromagnetic coupling substitution, $\boldsymbol{p} \rightarrow \boldsymbol{\pi}=\boldsymbol{p}+\boldsymbol{A} / \mathrm{c}$, where the canonical momentum of an electron $\boldsymbol{p}$ is substituted by the mechanical momentum $\pi$.

In relativistic electronic structure theory, the electromagnetic coupling manifests itself in two ways. First, the gauge transformation of the vector potential by means of a scalar function $\Lambda(\boldsymbol{r})$, $\boldsymbol{A} \rightarrow \boldsymbol{A}+\nabla \Lambda$, results in a phase change of the Dirac spinors. As advocated by London and Ditchfield, it is necessary to directly incorporate this phase change into a finite basis to achieve gauge origin independence of computational results and rapid convergence with basis set size. ${ }^{58,59}$ In general, the modification to an elemental basis function $f_{a}(\boldsymbol{r})(13)$ is

$$
f_{a}(\boldsymbol{r}) \rightarrow f_{a}(\boldsymbol{r}, \Lambda)=\exp \left[-\frac{i}{c} \Lambda_{a}\right] f_{a}(\boldsymbol{r})
$$

In the case of a vector potential due to a uniform external magnetic field $\boldsymbol{B}$, the gauge function $\Lambda_{a}$ has the following form:

$$
\Lambda_{a} \equiv \Lambda_{a}(\boldsymbol{r})=\frac{1}{2}\left[\boldsymbol{B} \times\left(\boldsymbol{R}_{a}-\boldsymbol{R}_{0}\right)\right] \cdot \boldsymbol{r},
$$

and shifts the vector potential from an arbitrary gauge-origin $\boldsymbol{R}_{0}$ to the origin $\boldsymbol{R}_{a}$ of $f_{a}$. As a result, the final magnetic field-dependent basis, often referred to as gauge-including atomic orbitals (GIAO) or London atomic orbitals (LAO), leads to gauge-origin independent results for molecular properties involving a uniform external magnetic field as perturbation. ${ }^{58,59}$ Second, the RKB coupling between the large- and small-component basis should also be substituted by a more general restricted magnetically balanced (RMB) operator ${ }^{32,34}$ in order to account for the effects of the minimal electromagnetic coupling substitution,

$$
\hat{\mathcal{O}}^{\mathrm{RKB}} \rightarrow \hat{\mathcal{O}}^{\mathrm{RMB}}=\frac{1}{2 c} \boldsymbol{\alpha} \cdot \boldsymbol{\pi}=\hat{\mathcal{O}}^{\mathrm{RKB}}+\frac{1}{2 c^{2}} \boldsymbol{\alpha} \cdot \boldsymbol{A} .
$$

In fact, the development and implementation of the concept of the RMB-GIAO basis was pioneered in the ReSpect program. ${ }^{32-34}$ In practice, this required the utilization of field-dependent GIAOs for 
the large-component basis spinors,

$$
\begin{aligned}
& \chi_{a}^{1}(\boldsymbol{r}, \Lambda):=\mathcal{N}_{a}^{\mathrm{L}}\left(\begin{array}{l}
1 \\
0 \\
0 \\
0
\end{array}\right) f_{a}(\boldsymbol{r}, \Lambda) \in \mathbb{C}^{4}\left(\mathbb{R}^{3}\right), \\
& \chi_{a}^{2}(\boldsymbol{r}, \Lambda):=\mathcal{N}_{a}^{\mathrm{L}}\left(\begin{array}{l}
0 \\
1 \\
0 \\
0
\end{array}\right) f_{a}(\boldsymbol{r}, \Lambda) \in \mathbb{C}^{4}\left(\mathbb{R}^{3}\right),
\end{aligned}
$$

embedded by an additional field-dependence through RMB for the small-component basis spinors $\chi_{a}^{3}, \chi_{a}^{4} \in \mathbb{C}^{4}\left(\mathbb{R}^{3}\right)$,

$$
\begin{aligned}
\chi_{a}^{3} & \equiv \chi_{a}^{3}(\boldsymbol{r}, \boldsymbol{A}, \Lambda)=\mathcal{N}_{a} \hat{\mathcal{O}}^{\mathrm{RMB}} \chi_{a}^{1}(\boldsymbol{r}, \Lambda) \\
& =\mathcal{N}_{a}^{S}\left[\left(\begin{array}{c}
0 \\
0 \\
-i \partial_{z} \\
\partial_{y}-i \partial_{x}
\end{array}\right)+\frac{1}{c}\left(\begin{array}{c}
0 \\
0 \\
A_{z} \\
A_{x}+i A_{y}
\end{array}\right)\right] f_{a}(\boldsymbol{r}, \Lambda), \\
\chi_{a}^{4} & \equiv \chi_{a}^{4}(\boldsymbol{r}, \boldsymbol{A}, \Lambda)=\mathcal{N}_{a} \hat{\mathcal{O}}^{\mathrm{RMB}} \chi_{a}^{2}(\boldsymbol{r}, \Lambda) \\
& =\mathcal{N}_{a}^{S}\left[\left(\begin{array}{c}
0 \\
0 \\
-\partial_{y}-i \partial_{x} \\
i \partial_{z}
\end{array}\right)+\frac{1}{c}\left(\begin{array}{c}
0 \\
0 \\
A_{x}-i A_{y} \\
-A_{z}
\end{array}\right)\right] f_{a}(\boldsymbol{r}, \Lambda) .
\end{aligned}
$$

Since ReSpect currently supports relativistic calculations of magnetic properties that require linear response functions to either a uniform external magnetic field or nuclear magnetic dipole moments, only the linear dependence of the basis spinors on these perturbations is required. Therefore, truncation of the exponential phase to first order, together with recasting the column vectors in Eqs. (29) and (30) into a matrix form similar to Eq. (18), results in the final expression for the RMB-GIAO basis-spinors,

$$
X_{a} \equiv X_{a}(\boldsymbol{r}, \boldsymbol{A}, \Lambda)=X_{a}^{\mathrm{RKB}}+X_{a}^{\mathrm{RMB}} \in \mathbb{C}^{4 \times 4}\left(\mathbb{R}^{3}\right) .
$$

The first RKB term $\left(X_{a}^{\mathrm{RKB}}\right)$ was already discussed in Sec. II B in terms of real quaternions and time-reversal symmetry [see Eq. (19)]. In contrast to RKB, the RMB term linear in $A$ and $\Lambda$ has a time-reversal antisymmetric (TRA) matrix structure,

$$
X_{a}^{\mathrm{RMB}} \equiv X_{a}^{\mathrm{RMB}}(\boldsymbol{r}, \boldsymbol{A}, \Lambda)=\left(\begin{array}{cc}
\mathcal{C} & \mathcal{D} \\
\mathcal{D}^{*} & -\mathcal{C}^{*}
\end{array}\right)_{a} \in \mathbb{C}_{-}^{4 \times 4}\left(\mathbb{R}^{3}\right),
$$

with elements

$$
\begin{aligned}
\mathcal{C}_{a}(\boldsymbol{r}, \boldsymbol{A}, \Lambda) & =\frac{1}{c}\left(\begin{array}{cc}
-i \Lambda_{a} f_{a}^{\mathrm{L}}(\boldsymbol{r}) & 0 \\
0 & \Lambda_{a, z}^{\mathrm{S}} f_{a}^{\mathrm{S}}(\boldsymbol{r})
\end{array}\right) \in \mathbb{C}^{2 \times 2}\left(\mathbb{R}^{3}\right), \\
\mathcal{D}_{a}(\boldsymbol{r}, \boldsymbol{A}, \Lambda) & =\frac{1}{c}\left(\begin{array}{cc}
0 & 0 \\
0 & \left(\Lambda_{a, x}^{\mathrm{S}}-i \Lambda_{a, y}^{\mathrm{S}}\right) f_{a}^{\mathrm{S}}(\boldsymbol{r})
\end{array}\right) \in \mathbb{C}^{2 \times 2}\left(\mathbb{R}^{3}\right), \\
\Lambda_{a, k}^{\mathrm{S}} & =A_{k}-\left(\partial_{k} \Lambda_{a}\right)-\Lambda_{a} \partial_{k}, \quad k \in x, y, z .
\end{aligned}
$$

Similarly to Eq. (21), it is possible to establish an algebraic isomorphism between the algebra of $4 \times 4$ complex TRA matrices and the algebra of $2 \times 2$ quaternion matrices over the field of pure imaginary numbers $i \mathbb{R}$, i.e., $\mathbb{C}_{-}^{4 \times 4} \simeq \mathbb{H}_{i \mathbb{R}}^{2 \times 2}$, such that for every $q \in \mathbb{C}_{-}^{4 \times 4}$, it holds that

$$
q=\left(\begin{array}{cc}
-q_{1}+i q_{0} & -q_{3}+i q_{2} \\
-q_{3}-i q_{2} & q_{1}+i q_{0}
\end{array}\right) \simeq i\left[q_{0}+q_{1} \mathbf{i}+q_{2} \mathbf{j}+q_{3} \mathbf{k}\right] \in \mathbb{H}_{i \mathbb{R}}^{2 \times 2},
$$

where $q_{0}, q_{1}, q_{2}, q_{3} \in \mathbb{R}^{2 \times 2}$. As a consequence, the RMB spinor basis in Eqs. (32) and (33) can be mapped onto a pure imaginary quaternion format

$$
X_{a}^{\mathrm{RMB}} \simeq{ }^{q} X_{a}^{\mathrm{RMB}}=i\left[{ }^{4} X_{a}+{ }^{5} X_{a} \mathbf{i}+{ }^{6} X_{a} \mathbf{j}+{ }^{7} X_{a} \mathbf{k}\right] \in \mathbb{H}_{i \mathbb{R}}^{2 \times 2}\left(\mathbb{R}^{3}\right)
$$

with the following quaternion constituents ${ }^{4-7} X_{a} \in \mathbb{R}^{2 \times 2}\left(\mathbb{R}^{3}\right)$ :

$$
\begin{gathered}
{ }^{4} X_{a}(\boldsymbol{r}, \boldsymbol{A}, \Lambda)=\Im\left(\mathcal{C}_{a}\right)=-\frac{1}{c}\left(\begin{array}{cc}
\Lambda_{a} & 0 \\
0 & 0
\end{array}\right) f_{a}^{\mathrm{L}}(\boldsymbol{r}), \\
{ }^{5} X_{a}(\boldsymbol{r}, \boldsymbol{A}, \Lambda)=-\mathfrak{R}\left(\mathcal{C}_{a}\right)=-\frac{1}{c}\left(\begin{array}{cc}
0 & 0 \\
0 & \Lambda_{a, z}^{\mathrm{S}}
\end{array}\right) f_{a}^{\mathrm{S}}(\boldsymbol{r}), \\
{ }^{6} X_{a}(\boldsymbol{r}, \boldsymbol{A}, \Lambda)=\Im\left(\mathcal{D}_{a}\right)=-\frac{1}{c}\left(\begin{array}{cc}
0 & 0 \\
0 & \Lambda_{a, y}^{\mathrm{S}}
\end{array}\right) f_{a}^{\mathrm{S}}(\boldsymbol{r}), \\
{ }^{7} X_{a}(\boldsymbol{r}, \boldsymbol{A}, \Lambda)=-\mathfrak{R}\left(\mathcal{D}_{a}\right)=-\frac{1}{c}\left(\begin{array}{cc}
0 & 0 \\
0 & \Lambda_{a, x}^{\mathrm{S}}
\end{array}\right) f_{a}^{\mathrm{S}}(\boldsymbol{r}) .
\end{gathered}
$$

The unconventional labels for constituents of 4-7 were selected on purpose, since it allows a uniform formulation of the general field-dependent RMB-GIAO spinor basis in Eq. (31) in the abstract algebra of complex quaternions $\mathbb{H}_{\mathbb{C}}^{2 \times 2}$ (also known as biquaternions),

$$
\begin{aligned}
X_{a}= & X_{a}(\boldsymbol{r}, \boldsymbol{A}, \Lambda) \simeq{ }^{q} X_{a}(\boldsymbol{r}, \boldsymbol{A}, \Lambda) \\
= & {\left[\left({ }^{0} X_{a}+i^{4} X_{a}\right)+\left({ }^{1} X_{a}+i^{5} X_{a}\right) \mathbf{i}+\left({ }^{2} X_{a}+i^{6} X_{a}\right) \mathbf{j}\right.} \\
& \left.+\left({ }^{3} X_{a}+i^{7} X_{a}\right) \mathbf{k}\right] \in \mathbb{H}_{\mathbb{C}}^{2 \times 2}\left(\mathbb{R}^{3}\right),
\end{aligned}
$$

with ${ }^{0-3} X_{a}$ and ${ }^{4-7} X_{a}$ as defined in Eqs. (25) and (36), respectively. Since an atomic basis of general symmetry can always be decomposed into the sum of TRS and TRA components, the abstract algebra of complex quaternions presented here is capable of covering all basis types with multicomponent character.

Note that the use of quaternion algebra for reducing the computational complexity of relativistic $4 \mathrm{c}$ calculations has already been advocated by several authors, with a primary focus either on matrix diagonalization $^{60-62}$ or on the handling of point group symmetry in a relativistic framework. ${ }^{63}$ All these approaches, however, are limited to Kramers-restricted (closed-shell) molecular cases and thus require only the algebra of real quaternions. In contrast, the present approach is based on a generalized concept of complex quaternions or biquaternions and formulates all basic ingredients such as basis and algebraic operators in a uniform formalism. In our experience, a significant reduction in memory and arithmetic operations can be achieved in this way. In ReSpect, complex quaternions have 
been applied in cases where the Kramers-unrestricted treatment is indispensable, such as theories involving open-shell species, ${ }^{29-31}$ real-time electron dynamics, ${ }^{37,41,42}$ molecular properties, ${ }^{33-35,39}$ or periodic systems. ${ }^{28}$

\section{Evaluation and processing of relativistic electron repulsion integrals (ERIs)}

One matrix quantity that plays a very important role in the design of efficient algorithms for the evaluation of relativistic electron repulsion integrals (ERIs) is the overlap distribution function $\mathbf{\Omega}$. In general, we define the matrix element $\Omega_{a b}$ of $\boldsymbol{\Omega}$ associated with the basis pair ${ }^{q} X_{a} \in \mathbb{H}_{\mathbb{C}}^{2 \times 2}\left(\mathbb{R}^{3}\right)$ and ${ }^{q} X_{b} \in \mathbb{H}_{\mathbb{C}}^{2 \times 2}\left(\mathbb{R}^{3}\right)$ as

$$
\Omega_{a b} \equiv \Omega_{a b}(\boldsymbol{r}, \boldsymbol{A}, \Lambda):={ }^{q} X_{a}^{\dagger}(\boldsymbol{r}, \boldsymbol{A}, \Lambda){ }^{q} X_{b}(\boldsymbol{r}, \boldsymbol{A}, \Lambda) \in \mathbb{H}_{\mathbb{C}}^{2 \times 2}\left(\mathbb{R}^{3}\right) .
$$

Here, ${ }^{q} X_{a}^{\dagger}$ denotes transposition and complex quaternion conjugation of the quaternion basis element ${ }^{q} X_{a}$ in Eq. (37). It is easy to prove that transposition and complex quaternion conjugation in $\mathbb{H}_{\mathbb{C}}^{2 \times 2}$ is equivalent to Hermitian conjugation in $\mathbb{C}^{4 \times 4}$. Conjugation of a complex quaternion results in a sign change in the pure part of $\mathbb{H}_{\mathbb{R}}^{2 \times 2}$ as well as in the real part of $\mathbb{H}_{i \mathbb{R}}^{2 \times 2}$.

The overlap distribution function composed of a simple RKB basis Eq. (24) has a time-reversal symmetric structure and thus belongs to $\mathbb{H}_{\mathbb{R}}^{2 \times 2}\left(\mathbb{R}^{3}\right)$. In this case, quaternion multiplication yields

$$
\begin{aligned}
\Omega_{a b}^{\mathrm{RKB}} & \equiv \Omega_{a b}^{\mathrm{RKB}}(\boldsymbol{r}):=\left({ }^{q} X_{a}^{\mathrm{RKB}}\right)^{\dagger} q^{\mathrm{RKB}} \\
& ={ }^{0} X_{a}{ }^{0} X_{b}+\left(\boldsymbol{X}_{a} \cdot \boldsymbol{X}_{b}\right)-\left(\boldsymbol{X}_{a} \times \boldsymbol{X}_{b}\right) \in \mathbb{H}_{\mathbb{R}}^{2 \times 2}\left(\mathbb{R}^{3}\right),
\end{aligned}
$$

where one can recognize the usual scalar and cross products in the vector space $\mathbb{R}^{3}$. In fact, the cross product is responsible for $\mathbb{H}$ being a non-commutative algebra. Note that we have followed here the compact notation for quaternions introduced in the last paragraph of Sec. II B. More importantly, there are only five non-zero scalar elements in the definition of the final quaternion constituents ${ }^{0-3} \Omega_{a b}^{\mathrm{RKB}} \in \mathbb{R}^{2 \times 2}\left(\mathbb{R}^{3}\right)$,

$$
\begin{aligned}
& { }^{0} \Omega_{a b}^{\mathrm{RKB}}={ }^{0} X_{a}{ }^{0} X_{b}+\left(\boldsymbol{X}_{a} \cdot \boldsymbol{X}_{b}\right)=\left(\begin{array}{cc}
f_{a}^{\mathrm{L}} f_{b}^{\mathrm{L}} & 0 \\
0 & \left(\boldsymbol{\partial} f_{a}^{\mathrm{S}}\right) \cdot\left(\boldsymbol{\partial} f_{b}^{\mathrm{S}}\right)
\end{array}\right), \\
& { }^{1} \Omega_{a b}^{\mathrm{RKB}}=-\left(\boldsymbol{X}_{a} \times \boldsymbol{X}_{b}\right)_{\mathbf{i}}=\left(\begin{array}{cc}
0 & 0 \\
0 & \epsilon_{z k l}\left(\partial_{k} f_{a}^{\mathrm{S}}\right)\left(\partial_{l} f_{b}^{\mathrm{S}}\right)
\end{array}\right), \\
& { }^{2} \Omega_{a b}^{\mathrm{RKB}}=-\left(\boldsymbol{X}_{a} \times \boldsymbol{X}_{b}\right)_{\mathbf{j}}=\left(\begin{array}{cc}
0 & 0 \\
0 & \epsilon_{y k l}\left(\partial_{k} f_{a}^{\mathrm{S}}\right)\left(\partial_{l} f_{b}^{\mathrm{S}}\right)
\end{array}\right), \\
& { }^{3} \Omega_{a b}^{\mathrm{RKB}}=-\left(\boldsymbol{X}_{a} \times \boldsymbol{X}_{b}\right)_{\mathbf{k}}=\left(\begin{array}{ll}
0 & 0 \\
0 & \epsilon_{x k l}\left(\partial_{k} f_{a}^{\mathrm{S}}\right)\left(\partial_{l} f_{b}^{\mathrm{S}}\right)
\end{array}\right)
\end{aligned}
$$

where $\epsilon$ is the Levi-Civita symbol and $k, l \in x, y, z$. As we shall discuss later, these five scalar elements form the fundamental building blocks of an algorithm for the efficient evaluation of relativistic electron repulsion integrals. In addition, as pointed out by Dyall, ${ }^{64}$ the small-small elements of the scalar and cross products in $\boldsymbol{\Omega}^{\mathrm{RKB}}$, when multiplied with a scalar multiplicative one-electron operator and integrated over $\mathbb{R}^{3}$, can be associated with contributions to the scalar and spin-orbit relativistic corrections, respectively.

Similarly to $\mathbf{\Omega}^{\mathrm{RKB}}$, one can define the overlap distribution function linear in a vector potential $\boldsymbol{A}$ and a gauge function $\Lambda$ as the quaternion product between RKB (24) and RMB (35) basis elements,

$$
\begin{aligned}
\Omega_{a b}^{\mathrm{RMB}} & \equiv \Omega_{a b}^{\mathrm{RMB}}(\boldsymbol{r}, \boldsymbol{A}, \Lambda):=\left({ }^{q} X_{a}^{\mathrm{RKB}}\right)^{\dagger}{ }^{\dagger} X_{b}^{\mathrm{RMB}} \\
& =i\left[{ }^{0} X_{a}{ }^{0} X_{b}+\left(\boldsymbol{X}_{a} \cdot \boldsymbol{X}_{b}\right)-\left(\boldsymbol{X}_{a} \times \boldsymbol{X}_{b}\right)\right] \in \mathbb{H}_{i \mathbb{R}}^{2 \times 2}\left(\mathbb{R}^{3}\right),
\end{aligned}
$$

$\mathbf{\Omega}^{\mathrm{RMB}}$ has the time-reversal antisymmetric structure inherited from the RMB basis, with the quaternion constituents consisting again only of five non-zero scalar elements,

$$
\begin{aligned}
{ }^{0} \Omega_{a b}^{\mathrm{RMB}} & ={ }^{0} X_{a}{ }^{0} X_{b}+\left(\boldsymbol{X}_{a} \cdot \boldsymbol{X}_{b}\right) \\
& =\frac{1}{c}\left(\begin{array}{cc}
-\Lambda_{b} f_{a}^{\mathrm{L}} f_{b}^{\mathrm{L}} & 0 \\
0 & \left(\boldsymbol{\partial} f_{a}^{\mathrm{S}}\right) \cdot\left(\boldsymbol{\Lambda}_{b}^{\mathrm{S}} f_{b}^{\mathrm{S}}\right)
\end{array}\right) \in \mathbb{R}^{2 \times 2}\left(\mathbb{R}^{3}\right), \\
{ }^{1} \Omega_{a b}^{\mathrm{RMB}} & =-\left(\boldsymbol{X}_{a} \times \boldsymbol{X}_{b}\right)_{\mathbf{i}} \\
& =\frac{1}{c}\left(\begin{array}{cc}
0 & 0 \\
0 & \epsilon_{z k l}\left(\partial_{k} f_{a}^{\mathrm{S}}\right)\left(\Lambda_{b, l}^{\mathrm{S}} f_{b}^{\mathrm{S}}\right)
\end{array}\right) \in \mathbb{R}^{2 \times 2}\left(\mathbb{R}^{3}\right), \\
{ }^{2} \Omega_{a b}^{\mathrm{RMB}} & =-\left(\boldsymbol{X}_{a} \times \boldsymbol{X}_{b}\right)_{\mathbf{j}} \\
& =\frac{1}{c}\left(\begin{array}{ll}
0 & \epsilon_{y k l}\left(\partial_{k} f_{a}^{\mathrm{S}}\right)\left(\Lambda_{b, l}^{\mathrm{S}} f_{b}^{\mathrm{S}}\right)
\end{array}\right) \in \mathbb{R}^{2 \times 2}\left(\mathbb{R}^{3}\right), \\
{ }^{3} \Omega_{a b}^{\mathrm{RMB}} & =-\left(\boldsymbol{X}_{a} \times \boldsymbol{X}_{b}\right)_{\mathbf{k}} \\
& =\frac{1}{c}\left(\begin{array}{ll}
0 & \epsilon_{x k l}\left(\partial_{k} f_{a}^{\mathrm{S}}\right)\left(\Lambda_{b, l}^{\mathrm{S}} f_{b}^{\mathrm{S}}\right)
\end{array}\right) \in \mathbb{R}^{2 \times 2}\left(\mathbb{R}^{3}\right),
\end{aligned}
$$

where $\Lambda^{S}$ is an operator related to the vector potential and gauge function and is defined in Eq. (33).

The discussed quaternion formalism of $\boldsymbol{\Omega}$ significantly reduces the time spent on evaluating and processing molecular integrals, since it identifies a unique nonredundant set of scalar integral components that enter into $4 \mathrm{c}$ theory. This formalism has therefore been encoded into ReSpect's integral library, called InteRest, which is based on the Obara-Saika integration technique over Cartesian Gaussians ${ }^{65}$ and allows the handling of various types of overlap distribution functions. For instance, the electron-nuclear Coulomb contribution to the Fock matrix can be computed as the simple product of a scalar multiplicative potential $\phi(\boldsymbol{r})$ and an arbitrary overlap distribution function $\Omega_{a b} \in \mathbb{H}_{\mathbb{C}}^{2 \times 2}\left(\mathbb{R}^{3}\right)$,

$$
J_{a b}^{\mathrm{n}}=-\sum_{A} Z_{A} \int \phi_{A}(\boldsymbol{r}) \Omega_{a b}(\boldsymbol{r}) d^{3} \boldsymbol{r} \in \mathbb{H}_{\mathbb{C}}^{2 \times 2} .
$$

Here, $\phi_{A}(\boldsymbol{r})$ is the external electrostatic scalar potential due to an atomic nucleus $A$, bearing the form either of a point-sized, Eq. (8), or of a finite-size nucleus model, Eq. (9).

The most computationally expensive part of InteRest, however, is associated with the evaluation $4 \mathrm{c}$ electron repulsion Coulomb integrals (ERIs), which, for a general $\Omega \in \mathbb{H}_{\mathbb{C}}^{2 n \times 2 n}\left(\mathbb{R}^{3}\right)$, reads as

$$
\left[\Omega_{a b} \mid \Omega_{c d}\right] \equiv \iint \frac{\Omega_{a b}\left(\boldsymbol{r}_{1}\right) \Omega_{c d}\left(\boldsymbol{r}_{2}\right)}{r_{12}} d^{3} \boldsymbol{r}_{1} d^{3} \boldsymbol{r}_{2} \in \mathbb{H}_{\mathbb{C}}^{2 \times 2} \otimes \mathbb{H}_{\mathbb{C}}^{2 \times 2}
$$


Here, $\otimes$ denotes the tensor product and $r_{12}=\left|\boldsymbol{r}_{1}-\boldsymbol{r}_{2}\right|$. In the simplest case of $\boldsymbol{\Omega}=\boldsymbol{\Omega}^{\mathrm{RKB}}$ and/or $\boldsymbol{\Omega}=\boldsymbol{\Omega}^{\mathrm{RMB}}$, a single $4 \mathrm{c}$ ERI requires the evaluation and processing of 25 times more real scalar integrals than the non-relativistic case. This should be compared with the factor of 100 presented by some authors and obtained by incorrectly assuming that the small-component scalar functions $f_{a}^{S}$ are simply derived for the RKB case as $f_{a}^{\mathrm{S}}=\left\{\partial_{x} f_{a}^{\mathrm{L}}, \partial_{y} f_{a}^{\mathrm{L}}, \partial_{z} f_{a}^{\mathrm{L}}\right\}$ and are used in this form to evaluate all integral classes: [LL|LL], [SS|LL], [LL|SS], and [SS|SS]. In addition, the size of the integrals in these classes decays in powers of $c^{-2}$ and thus gradually decreases along the series

$$
[\mathrm{LL} \mid \mathrm{LL}]>[\mathrm{LL} \mid \mathrm{SS}] \sim[\mathrm{SS} \mid \mathrm{LL}]>[\mathrm{SS} \mid \mathrm{SS}] .
$$

Therefore, it is reasonable to exclude some computationally intensive [SS|SS] contributions that are of the order $\mathcal{O}\left(c^{-4}\right)$. For this purpose, InteRest enables the use of the so-called one-center approximation for [SS|SS], where all integrals from this class are discarded unless the scalar functions defining [SS| (bra) as well as the scalar functions defining $\mid \mathrm{SS}]$ (ket) share the same origin. This approximation is now the program's default and leads to a speed-up of the ERI evaluation by a factor of 2-3 for $\Omega^{\mathrm{RKB}}$ while introducing negligible errors of about $10^{-5}$ Hartree in the total self-consistent field (SCF) energies. Note that in contrast to some other approaches, ${ }^{66}$ the onecenter approximation treats the [SS|SS] integrals self-consistently when evaluating the Coulomb and exchange Fock contributions and does not rely on any pre-calculated small-component atomic densities.

Let us conclude this section by defining a general algebraic representation of the two-electron Coulomb interaction operator $(\mathbf{G})$. In the 4c DKS framework, as implemented in ReSpect, $\mathbf{G}$ includes both the two-electron Coulomb $\left(\mathbf{J}^{\mathrm{e}}\right)$ and exchange $(\mathbf{K})$ contributions, with the elements

$$
G_{a b}=J_{a b}^{\mathrm{e}}+\xi K_{a b} \in \mathbb{H}_{\mathbb{C}}^{2 \times 2},
$$

where $\xi \in \mathbb{R}$ is a scaling parameter associated with hybrid exchangecorrelation functionals. For pure DFT, $\xi=0$, whereas for hybrid DFT, $0<\xi<1$. In the most general case of a one-electron reduced density matrix $\mathbf{D} \in \mathbb{H}_{\mathbb{C}}^{2 n \times 2 n}$, the Coulomb and exchange interaction terms are given by the expressions

$$
\begin{aligned}
J_{a b}^{\mathrm{e}} & =\left[\Omega_{a b} \mid \operatorname{Tr}\left\{\Omega_{c d} D_{d c}\right\}\right] \\
& =\iint \Omega_{a b}\left(\boldsymbol{r}_{1}\right) \frac{1}{r_{12}} \operatorname{Tr}\left\{\Omega_{c d}\left(\boldsymbol{r}_{2}\right) D_{d c}\right\} d^{3} \boldsymbol{r}_{1} d^{3} \boldsymbol{r}_{2} \in \mathbb{H}_{\mathbb{C}}^{2 \times 2}, \\
K_{a b} & =\left[\Omega_{a d}\left|D_{d c}\right| \Omega_{c b}\right] \\
& =\iint \Omega_{a d}\left(\boldsymbol{r}_{1}\right) \frac{D_{d c}}{r_{12}} \Omega_{c b}\left(\boldsymbol{r}_{2}\right) d^{3} \boldsymbol{r}_{1} d^{3} \boldsymbol{r}_{2} \in \mathbb{H}_{\mathbb{C}}^{2 \times 2} .
\end{aligned}
$$

Here, $\operatorname{Tr}$ denotes a trace in $\mathbb{H}_{\mathbb{C}}^{2 \times 2}$,

$$
\operatorname{Tr}\{\}: \mathbb{H}_{\mathbb{C}}^{2 \times 2} \rightarrow \mathbb{C},
$$

whose final values depend on the time-reversal symmetry of the argument. For $q \in \mathbb{H}_{\mathbb{R}}^{2 \times 2}$ in Eq. (21), $\operatorname{Tr}\{q\}=2\left({ }^{0} q_{11}+{ }^{0} q_{22}\right)$, whereas for $q \in \mathbb{H}_{i \mathbb{R}}^{2 \times 2}$ in Eq. (34), $\operatorname{Tr}\{q\}=2 i\left({ }^{4} q_{11}+{ }^{4} q_{22}\right)$. It is important to emphasize that the non-commutative nature of $\mathbb{H}$ forbids any rearrangement of the density and overlap distribution matrices in the two-electron exchange expression (47). This also holds for complex algebra in both the $2 \mathrm{c}$ and $4 \mathrm{c}$ frameworks.
In addition to the exact treatment of ERIs, all property modules of ReSpect are also equipped with an approximate ERI evaluation technique based on the resolution of the identity (RI). ${ }^{67}$ In the sense of Dunlap's robust fit, ${ }^{68} \mathrm{RI}$ allows the approximation of the exact ERIs in Eq. (44) by $\left[\overline{\Omega_{a b} \mid \Omega_{c d}}\right]$ in such a way that the residual Coulomb-repulsion integral,

$$
\left[\Delta \Omega_{a b} \mid \Delta \Omega_{c d}\right]=\left[\Omega_{a b} \mid \Omega_{c d}\right]-\left[\overline{\Omega_{a b} \mid \Omega_{c d}}\right],
$$

is bilinear in the errors $\Delta \Omega_{a b}$ and $\Delta \Omega_{c d}$. It is customary to approximate the overlap distributions $\left.\mid \Omega_{a b}\right]$ by a superposition of real, scalar, atom-centered auxiliary basis functions $\mid \alpha]$. We can then write

$$
\left.\left.\left.\mid \Delta \Omega_{a b}\right]=\mid \Omega_{a b}\right]-c_{\alpha}^{a b} \mid \alpha\right] .
$$

The individual expansion coefficients $c_{\alpha}^{a b}$ are then obtained by minimizing the residual Coulomb-repulsion integral with respect to $c_{\alpha}^{a b}$. This leads to a set of linear equations

$$
[\alpha \mid \beta] c_{\beta}^{a b}=\left[\alpha \mid \Omega_{a b}\right],
$$

whose solution, when inserted into the expression for $\mathbf{J}^{\mathrm{e}}$ with approximate integrals, gives

$$
J_{a b}^{\mathrm{e}} \approx\left[\Omega_{a b} \mid \alpha\right][\alpha \mid \beta]^{-1}\left[\beta \mid \operatorname{Tr}\left\{\Omega_{c d} D_{d c}\right\}\right] .
$$

An additional efficiency gain yielded by $4 \mathrm{c}$ RI, not present for its non-relativistic counterpart, results from the possibility of using an identical auxiliary basis for expanding both the large- and smallcomponent overlap distributions. This approach has been successfully examined in 4c SCF optimizations ${ }^{69-71}$ as well as in property calculations in the static ${ }^{70,72}$ and the time domain.

\section{E. Noncollinear DFT potential and kernel}

To fully capture spin-orbit coupling effects and to ensure rotational invariance of the total energy in the relativistic framework, the standard non-relativistic collinear parameterization of DFT functionals is no longer adequate. In this section, we shall describe the different strategies by which we seek to remedy this problem within the generalized gradient approximation of DFT.

In this subsection, we assume the following: complex algebra, the four-component flattened atomic orbital basis indices $\mu, v, \lambda$, and $\tau$ (for more details, see Ref. 39), and if not otherwise specified, we indicate either matrix or vector quantities, depending on the context, by bold font. In addition, Einstein's implicit summation over repeated indices is assumed. Furthermore, we use the auxiliary variables $v^{t}$ and $k^{t w}$,

$$
v^{t}=\frac{\partial \varepsilon^{\mathrm{xc}}}{\partial t}, \quad k^{t w}=\frac{\partial \varepsilon^{\mathrm{xc}}}{\partial t \partial w},
$$

where $\varepsilon^{\mathrm{xc}}$ represents the exchange-correlation energy density. The charge, $\rho_{0}$, and the spin density, $\rho$, variables have the form

$$
\begin{gathered}
\rho_{0}=\left(\boldsymbol{X}_{\mu}^{\mathrm{RKB}}\right)^{\dagger} \boldsymbol{X}_{v}^{\mathrm{RKB}} D_{v \mu}, \\
\rho_{k}=\left(\boldsymbol{X}_{\mu}^{\mathrm{RKB}}\right)^{\dagger} \boldsymbol{\Sigma}_{k} \boldsymbol{X}_{v}^{\mathrm{RKB}} D_{v \mu},
\end{gathered}
$$


where $\Sigma_{k}$ is the four-component matrix composed of Pauli matrices,

$$
\boldsymbol{\Sigma}_{k}=\left(\begin{array}{cc}
\boldsymbol{\sigma}_{k} & \mathbf{0} \\
\mathbf{0} & \boldsymbol{\sigma}_{k}
\end{array}\right), \quad k=1,2,3,
$$

and the four-component overlap distributions are defined as

$$
\Omega_{\mu \nu}^{m}=\frac{\partial \rho_{m}}{\partial D_{v \mu}}, \quad \nabla \Omega_{\mu \nu}^{m}=\frac{\partial\left(\nabla \rho_{m}\right)}{\partial D_{v \mu}}, \quad m=0, \ldots, 4 .
$$

\section{Noncollinear exchange-correlation energy}

In the case of a time-reversal symmetric density matrix (closedshell Kramers-restricted KS determinant), the spin density and its gradient vanish. The exchange-correlation energy then has the particularly simple form

$$
E^{\mathrm{xc}, \mathrm{cs}}=\int \mathcal{E}^{\mathrm{xc}}\left[\rho_{0}, \nabla \rho_{0}\right] d^{3} r .
$$

In the case of a density matrix without any time-reversal symmetry (an open-shell Kramers-unrestricted KS determinant), the situation is more complex. In the non-relativistic (nr) spin-density functional theory (SDFT), functionals of the generalized-gradient approximation (GGA) are parameterized in terms of the electron charge density and the $z$ component of the spin density. The exchange-correlation contribution to the molecular energy is then defined as

$$
E^{\mathrm{xc}, \mathrm{nr}}=\int \varepsilon^{\mathrm{xc}}\left[\rho_{0}, \nabla \rho_{0} \cdot \nabla \rho_{0}, \rho_{z}, \nabla \rho_{z} \cdot \nabla \rho_{z}, \nabla \rho_{0} \cdot \nabla \rho_{z}\right] d^{3} \boldsymbol{r}
$$

or

$$
E^{\mathrm{xc}, \mathrm{nr}}=\int \varepsilon^{\mathrm{xc}}\left[\rho_{0}, \nabla \rho_{0}, \rho_{z}, \nabla \rho_{z}\right] d^{3} \boldsymbol{r} .
$$

However, the parameterization of the exchange-correlation energy density, which depends only on the $\mathrm{z}$ component of the electron spin density, is inadequate for theories that include spin-orbit coupling effects. In such cases, the spatial and spin variables are not independent, and the parameterization with collinear variables in Eq. (59) or Eq. (60) gives results that lack rotational invariance with respect to the rotation of the coordinate axis system.

To circumvent this problem, we employ the so-called noncollinear ansatz, i.e., all functionals in the ReSpect program are borrowed from collinear SDFT, with the collinear variables replaced by the corresponding noncollinear ones. We use two different noncollinear schemes for the calculation of the exchange-correlation energy.

The first approach utilizes a technique where the $\mathrm{z}$ component of the spin density, $\rho_{z}$, is replaced by its magnitude, $|\rho|$ (see Ref. 73); this is directly extended to GGA functionals by replacing the gradient of $\rho_{z}$ with the gradient of $|\rho|$,

$$
\begin{gathered}
\rho_{z} \rightarrow|\boldsymbol{\rho}|, \\
\nabla \rho_{z} \rightarrow \nabla|\boldsymbol{\rho}| .
\end{gathered}
$$

Although the extension to GGA functionals in Eq. (62) results in numerical instabilities in the evaluation of noncollinear DFT potentials and kernels, it causes no problems for the calculation of the exchange-correlation energy. Using the noncollinear variables in
Eqs. (61) and (62), the exchange-correlation energy has the form

$$
E^{\mathrm{xc}, \mathrm{ut}}=\int \mathcal{E}^{\mathrm{xc}}[n, \nabla n, s, \nabla s] d^{3} \boldsymbol{r},
$$

where we define the auxiliary variables as $n \equiv \rho_{0}$ and $s \equiv|\rho|$.

The second approach is based on the noncollinear ansatz introduced by Scalmani and Frisch, ${ }^{74}$ which substitutes the variables in Eq. (59) depending on the $\mathrm{z}$ quantization axis by more adequate rotationally invariant variables,

$$
\begin{aligned}
\rho_{z} & \rightarrow s, \\
\nabla \rho_{0} \cdot \nabla \rho_{z} & \rightarrow f_{\nabla} g, \\
\nabla \rho_{z} \cdot \nabla \rho_{z} & \rightarrow \nabla \rho_{k} \cdot \nabla \rho_{k},
\end{aligned}
$$

where $k=1,2,3, g \equiv|g|$, and $\boldsymbol{g}$ and $f_{\nabla}$ are defined as

$$
\begin{aligned}
& g_{k}=\nabla \rho_{0} \cdot \nabla \rho_{k}, \\
& f_{\nabla}=\operatorname{sgn}(\boldsymbol{g} \cdot \boldsymbol{\rho}) .
\end{aligned}
$$

The noncollinear exchange-correlation energy then becomes

$$
E^{\mathrm{xc}, \mathrm{sf}}=\int \varepsilon^{\mathrm{xc}}\left[n, \nabla n \cdot \nabla n, s, \nabla \rho_{k} \cdot \nabla \rho_{k}, f_{\nabla} g\right] d^{3} r .
$$

\section{Noncollinear exchange-correlation potential}

Here, again, we consider two cases: a time-reversal symmetric density matrix (a closed-shell Kramers-restricted KS determinant) and a density matrix without any time-reversal symmetry (an open-shell Kramers-unrestricted KS determinant). In the first case, the spin density and its gradient are zero everywhere, and the noncollinear DFT potential has the form $(l=1,2,3)$

$$
V_{\mu \nu}^{\mathrm{xc}, \mathrm{cs}}=\frac{\mathrm{d} E^{\mathrm{xc}, \mathrm{cs}}}{\mathrm{d} D_{\nu \mu}}=\int\left(v^{n} \Omega_{\mu \nu}^{0}+v^{\nabla_{l} n} \nabla_{l} \Omega_{\mu \nu}^{0}\right) d^{3} \boldsymbol{r} .
$$

In the second case, the situation is more challenging because of the numerical instabilities ${ }^{39,74}$ that affect the GGA part of the noncollinear DFT functionals when the noncollinear ansatz for gradients of the spin density described in Eq. (62) is used. There are two approaches implemented in the ReSpect program, which resolve this issue.

The first approach rotates the local axis system at each point in space in such a way that the $z$ axis is aligned with the direction of the spin density, $\boldsymbol{\rho}(\boldsymbol{r})$. In this transformed axis system, the standard collinear SDFT potential is first calculated and then rotated back to the original axis system using a unitary matrix $\mathbf{U}(\boldsymbol{r})$ defined by the vector $\boldsymbol{\rho}(\boldsymbol{r})$. For a more detailed discussion, see, for example, Refs. 44, 75 , and 76. After this procedure, the noncollinear DFT potential has the form

$$
\begin{aligned}
V_{\mu \nu}^{\mathrm{xc}, \mathrm{ut}} & =\frac{\mathrm{d} E^{\mathrm{xc}, \mathrm{ut}}}{\mathrm{d} D_{\nu \mu}} \\
& \approx \int\left(v^{n} \Omega_{\mu \nu}^{0}+v^{\nabla_{l} n} \nabla_{l} \Omega_{\mu \nu}^{0}+v^{s} \frac{\rho_{k}}{s} \Omega_{\mu \nu}^{k}+v^{\nabla_{l} s} \frac{\rho_{k}}{s} \nabla_{l} \Omega_{\mu \nu}^{k}\right) d^{3} \boldsymbol{r},
\end{aligned}
$$

with $k, l=1,2,3$. Note that the first three terms in Eq. (68) are variational, i.e., they arise from the chain rule involving the variables $n, \nabla_{l} n$, and $s$ when evaluating the derivative of the noncollinear 
exchange-correlation energy [Eq. (63)] with respect to the density matrix $\mathbf{D}$. Because the same rotational matrix $\mathbf{U}(\boldsymbol{r})$ is used for the back-transformation of both collinear functions $v^{\rho_{z}}$ and $v^{\nabla / \rho_{z}}$, the last term in Eq. (68) is not variational, and thus, the second equality in Eq. (68) is only approximate. Another problem is that all functions $v^{t}$ in Eq. (68) are evaluated as $v^{t}\left(\nabla_{l} s\right)$, where $\nabla_{l} s$ is a function describing only the longitudinal change of the spin density by projecting the vector $\nabla_{l} \rho$ onto the local direction of $\rho$, and therefore, the important transverse components of the gradients $\nabla_{l} \rho$ are neglected.

The second approach resolving numerical instabilities for open-shell noncollinear DFT potentials is based on the noncollinear variables defined in Eq. (64). In this case, the exchange-correlation potential has the form

$$
\begin{aligned}
V_{\mu \nu}^{\mathrm{xc}, \mathrm{sf}}= & \frac{\mathrm{d} E^{\mathrm{xc}, \mathrm{sf}}}{\mathrm{d} D_{v \mu}} \\
= & \int\left(v^{n} \Omega_{\mu v}^{0}+v^{s} \frac{\rho_{k}}{s} \Omega_{\mu v}^{k}+v^{g_{n n}} 2 \nabla_{l} \rho_{0} \nabla_{l} \Omega_{\mu v}^{0}\right. \\
& +v^{g_{s s}} 2 \nabla_{l} \rho_{k} \nabla_{l} \Omega_{\mu \nu}^{k}+v^{g_{n s}} f_{\nabla} \frac{g_{k}}{g} \nabla_{l} \rho_{k} \nabla_{l} \Omega_{\mu v}^{0} \\
& \left.+v^{g_{n s}} f_{\nabla} \frac{g_{k}}{g} \nabla_{l} \rho_{0} \nabla_{l} \Omega_{\mu \nu}^{k}\right) d^{3} \boldsymbol{r},
\end{aligned}
$$

where $g_{n n} \equiv \nabla \rho_{0} \cdot \nabla \rho_{0}, g_{n s} \equiv f_{\nabla} g$, and $g_{s s} \equiv \nabla \rho_{k} \cdot \nabla \rho_{k}$. We pay special attention to the evaluation of the exchange-correlation potential in Eq. (69) in the limiting cases where the $s$ or $g$ functions approach zero. A detailed description of this procedure is given in Ref. 39.

\section{Noncollinear exchange-correlation kernel}

In the case of the closed-shell Kramers-restricted reference state, there are three types of noncollinear exchange-correlation kernels available in the ReSpect program. We recommend using the following formulation, ${ }^{78}$ because of its good performance in LR-TDDFT calculations:

$$
\begin{aligned}
K_{\mu \nu, \lambda \tau}^{\mathrm{xc}, \mathrm{cs}} \widetilde{D}_{\tau \lambda}= & \frac{\mathrm{d} V_{\mu \nu}^{\mathrm{xcccs}}}{\mathrm{d} D_{\tau \lambda}} \widetilde{D}_{\tau \lambda} \\
= & \int\left\{\left(k^{n n} \tilde{\rho}_{0}+k^{n \nabla_{l} n} \nabla_{l} \tilde{\rho}_{0}\right) \Omega_{\mu \nu}^{0}\right. \\
& +\left(k^{n \nabla_{m} n} \tilde{\rho}_{0}+k^{\nabla_{l} n \nabla_{m} n} \nabla_{l} \tilde{\rho}_{0}\right) \nabla_{m} \Omega_{\mu \nu}^{0} \\
& +\left(k^{s s} \tilde{\rho}_{k}+k^{s \nabla_{l}^{s}} \nabla_{l} \tilde{\rho}_{k}\right) \Omega_{\mu \nu}^{k} \\
& \left.+\left(k^{s \nabla_{m} s} \tilde{\rho}_{k}+k^{\nabla_{l} s \nabla_{m} s} \nabla_{l} \tilde{\rho}_{k}\right) \nabla_{m} \Omega_{\mu \nu}^{k}\right\} d^{3} \boldsymbol{r},
\end{aligned}
$$

where $\tilde{\rho}_{0}$ and $\tilde{\rho}$ are the induced charge and spin density, respectively. Note that if the perturbation is magnetic, the use of the magnetically balanced basis in the evaluation of $\tilde{\rho}_{0}$ and $\tilde{\rho}$ is mandatory (see Sec. VI).

The second option is the exchange-correlation kernel used until now in all applications of the ReSpect program (with the analytical evaluation of the kernel) to the prediction of NMR chemical shifts, indirect nuclear spin-spin coupling constants, and nuclear spin rotation constants,

$$
\begin{aligned}
K_{\mu \nu, \lambda \tau}^{\mathrm{xc}, \mathrm{ut}} \widetilde{D}_{\tau \lambda}= & \int\left(k^{s s} \tilde{\rho}_{k} \Omega_{\mu \nu}^{k}+k^{s \nabla_{l} s} \tilde{\rho}_{k} \nabla_{l} \Omega_{\mu \nu}^{k}+k^{s \nabla_{l}^{s}} \frac{\nabla_{l}|\tilde{\boldsymbol{\rho}}|}{\tilde{\boldsymbol{\rho}}} \tilde{\rho}_{k} \Omega_{\mu v}^{k}\right. \\
& \left.+k^{\nabla_{l} s \nabla_{m} s} \frac{\nabla_{l}|\tilde{\boldsymbol{\rho}}|}{|\tilde{\boldsymbol{\rho}}|} \tilde{\rho}_{k} \nabla_{m} \Omega_{\mu \nu}^{k}\right) d^{3} \boldsymbol{r}
\end{aligned}
$$

The formulation of Eq. (71) follows the same procedure as used in the derivation of the exchange-correlation potential (68), where the local unitary transformation, $\mathbf{U}(\boldsymbol{r})$, is defined by the orientation of the spin vector $\tilde{\boldsymbol{\rho}}{ }^{44}$ However, it was recently shown that the extension of this formulation to the calculation of excitation energies gives unsatisfactory results. ${ }^{39}$ Although so far all test calculations performed for magnetic properties have shown negligible differences between the results obtained by the exchange-correlation kernels Eqs. (70) and (71), we recommend reconsidering the use of the latter and we keep it mostly for the reproducibility of previously published scientific data.

The third option is to use an approximation where the gradients of the charge and spin density are neglected in the evaluation of the xc kernel for the closed-shell reference KS determinant, regardless of the type of the DFT functional used. This approximation is known as the adiabatic local density approximation (ALDA). The use of the ALDA kernel is not recommended in production calculations, ${ }^{32}$ although it is useful for evaluating the effect of the approximation on the final results, and for comparison with other published results using this approximation. The ALDA noncollinear exchange-correlation kernel has the simple form

$$
K_{\mu v, \lambda \tau}^{\mathrm{xc}, \mathrm{alda}} \widetilde{D}_{\tau \lambda}=\int\left\{k^{n n} \tilde{\rho}_{0} \Omega_{\mu \nu}^{0}+k^{s s} \tilde{\rho}_{k} \Omega_{\mu \nu}^{k}\right\} d^{3} \boldsymbol{r} .
$$

Finally, for the case of the open-shell Kramers-unrestricted reference state, we have recently implemented a noncollinear exchange-correlation kernel that is consistent with the xc energy equation [Eq. (66)] and xc potential equation [Eq. (69)]. ${ }^{39}$ Expressions for this noncollinear xc kernel are too lengthy to be compactly presented in this work. In Ref. 39, we paid special attention to the proper behavior of the noncollinear potential and kernel in the limiting cases where the length of the $\boldsymbol{\rho}$ or $\boldsymbol{g}$ vectors approaches zero. As a result, an important difference in the ReSpect implementation as compared to other approaches is its increased numerical stability, improved rotational invariance in the limiting cases, guaranteed closed-shell limit, and treatment of systems with non-degenerate and degenerate ground states on an equal footing.

\section{MOLECULAR SCF}

In the $4 c$ relativistic Dirac-Kohn-Sham (DKS) DFT theory, the SCF working equation for molecular systems takes the matrix form

$$
\mathbf{F C}=\mathbf{S C} \epsilon,
$$

where $\mathbf{F}$ and $\mathbf{S}$ are the Fock and overlap matrices, respectively, $\mathbf{C}$ is the matrix of molecular orbital/spinor coefficients, and $\epsilon$ is a diagonal matrix consisting of MO energies. For a scalar basis of size $n$, the DKS equation is of size $4 n \times 4 n$ and can be solved either in the complex algebra $\mathbb{C}^{4 n \times 4 n}$ or in the quaternion algebra $\mathbb{H}^{2 n \times 2 n}$. ReSpect utilizes the former option as it greatly benefits from existing optimized and parallel math libraries such as 
LAPACK/BLAS. The necessity of using special libraries highlights the fact that the eigenvalue problem, which scales nearly cubically with the system size, is 32 times larger than its non-relativistic counterpart. Therefore, diagonalization can easily become bottlenecks in our $4 \mathrm{c}$ calculations even for systems of medium size ( $\sim 50$ atoms), in particular, when utilizing the RI approach for Fock matrix builds. Various attempts have been reported in the literature to reduce the computational burden associated with the solution of the $4 \mathrm{c}$ eigenvalue problem, such as the diagonalization-free approach implemented in ReSpect. ${ }^{79}$ In the case of the two-component X2C Hamiltonian, the eigenvalue equation is only $2 n \times 2 n$ in size, and hence, its solution poses a lesser computational challenge than the full $4 \mathrm{c}$ treatment.

Additional computational complexity arises in 4c SCF in the evaluation of the overlap and Fock operators in the RKB basis. Here, ReSpect takes the full advantage of the quaternion algebra that is explicitly built into the program's integral library InteRest. The overlap matrix $\mathbf{S} \in \mathbb{C}_{+}^{4 n \times 4 n}$ in Eq. (73) is thus obtained from its quaternion isomorphic image ${ }^{q} \mathbf{S} \in \mathbb{H}_{\mathbb{R}}^{2 n \times 2 n}$,

$$
S_{a b} \simeq{ }^{q} S_{a b}=\int_{\mathbb{R}^{3}} \Omega_{a b}(\boldsymbol{r}) d^{3} \boldsymbol{r} \in \mathbb{H}_{\mathbb{R}}^{2 \times 2} .
$$

Obviously, ${ }^{q} S_{a b}$ has non-zero elements only in its quaternion real part when evaluated from the RKB overlap distribution function ${ }^{q} \Omega_{a b}$ [see also Eq. (39)]. Evaluation of the Fock matrix, on the other hand, is significantly more elaborate and depends on the TR symmetry of the one-electron reduced density matrix $\mathbf{D}=\mathbf{C f C}^{\dagger}$. Here, $\mathbf{f}$ stands for a diagonal matrix of occupation numbers.

In the absence of an external electromagnetic potential, the density matrix of closed-shell molecular systems is Hermitian and TR-symmetric, i.e., $\mathbf{D}=\mathbf{D}^{\dagger} \in \mathbb{C}_{+}^{4 n \times 4 n}$. In the RKB basis, the Fock matrix also obeys the same algebraic properties as $\mathbf{D}$, and solutions of Eq. (73) come in degenerate Kramers pairs. We shall commonly refer to this case as being Kramers-restricted (KR). In actual KR calculations, $\mathbf{F} \in \mathbb{C}_{+}^{4 n \times 4 n}$ is obtained from its quaternion isomorphic image ${ }^{q} \mathbf{F} \in \mathbb{H}_{\mathbb{R}}^{2 n \times 2 n}$ with the elements

$$
\begin{aligned}
F_{a b} & \simeq{ }^{q} F_{a b}={ }^{0} F_{a b}+F_{a b} \\
& ={ }^{0} F_{a b}+{ }^{1} F_{a b} \mathbf{i}+{ }^{2} F_{a b} \mathbf{j}+{ }^{3} F_{a b} \mathbf{k} \in \mathbb{H}_{\mathbb{R}}^{2 \times 2} .
\end{aligned}
$$

Here, the real and pure quaternion parts read

$$
\begin{aligned}
{ }^{0} F_{a b} & ={ }^{0} T_{a b}+{ }^{0} J_{a b}^{\mathrm{n}}+{ }^{0} J_{a b}^{\mathrm{e}}\left[{ }^{q} \mathbf{D}\right]+{ }^{0} K_{a b}\left[{ }^{q} \mathbf{D}\right]+{ }^{0} V_{a b}^{\mathrm{xc}}\left[{ }^{q} \mathbf{D}\right], \\
\boldsymbol{F}_{a b} & =\boldsymbol{J}_{a b}^{\mathrm{n}}+\boldsymbol{J}_{a b}^{\mathrm{e}}\left[{ }^{q} \mathbf{D}\right]+\boldsymbol{K}_{a b}\left[{ }^{q} \mathbf{D}\right]+\boldsymbol{V}_{a b}^{\mathrm{xc}}\left[{ }^{q} \mathbf{D}\right],
\end{aligned}
$$

where $T_{a b}, J_{a b}^{\mathrm{n}}, J_{a b}^{\mathrm{e}}$, and $K_{a b}$ are the matrix elements of the kinetic, nuclear-electron and electron-electron Coulomb, and HartreeFock exchange energy operators introduced in Eqs. (43) and (47), respectively. In the context of the SCF discussion, both ${ }^{q} \Omega_{a b}$ and ${ }^{q} \Omega_{c d}$ in Eq. (47) refer to the RKB basis as defined by Eq. (39). The last term in Eq. (76) refers to the DFT noncollinear exchangecorrelation potential, details of which have already been discussed in Sec. II E 2.

For open-shell molecular systems, ReSpect supports the Kramers-unrestricted (KU) formalism in which the time-reversal symmetry is no longer preserved and Kohn-Sham solutions do not consist of degenerate Kramers partners. This effect is known in non-relativistic theory as spin polarization and plays an essential role in calculations of molecular core-level properties for open-shell species, for instance, the EPR hyperfine coupling tensor. In the KU approach, both the density matrix and the Fock matrix are Hermitian and TR-general, and hence, the algebra of complex quaternions $\mathbb{H}_{\mathbb{C}}^{2 n \times 2 n}$ is required for their representation. Similarly to Eq. (37), ReSpect calculates the KU Fock matrix elements $F_{a b} \in \mathbb{C}^{4 \times 4}$ by means of their quaternion isomorphic images ${ }^{q} F_{a b} \in \mathbb{H}_{\mathbb{C}}^{2 \times 2}$,

$$
F_{a b} \simeq{ }^{q} F_{a b}={ }^{q} F_{a b}^{+}+{ }^{q} F_{a b}^{-}={ }^{0} F_{a b}^{+}+{ }^{0} F_{a b}^{-}+F_{a b}^{+}+F_{a b}^{-} .
$$

The evaluation of the TR-symmetric Fock contribution ${ }^{q} F_{a b}^{+} \in \mathbb{H}_{\mathbb{R}}^{2 \times 2}$ proceeds similarly to the KR case [Eq. (76)] and involves only the TR-symmetric component of the density matrix ${ }^{q} \mathbf{D}^{+} \in \mathbb{H}_{\mathbb{R}}^{2 n \times 2 n}$, except for the exchange-correlation contribution, which depends on the density matrix of both TR symmetries,

$$
\begin{aligned}
{ }^{0} F_{a b}^{+} & ={ }^{0} T_{a b}+{ }^{0} J_{a b}^{\mathrm{n}}+{ }^{0} J_{a b}^{\mathrm{e}}\left[{ }^{q} \mathbf{D}^{+}\right]+{ }^{0} K_{a b}\left[{ }^{q} \mathbf{D}^{+}\right]+{ }^{0} V_{a b}^{\mathrm{xc}+}\left[{ }^{q} \mathbf{D}\right], \\
\boldsymbol{F}_{a b}^{+} & =\boldsymbol{J}_{a b}^{\mathrm{n}}+\boldsymbol{J}_{a b}^{\mathrm{e}}\left[{ }^{q} \mathbf{D}^{+}\right]+\boldsymbol{K}_{a b}\left[{ }^{q} \mathbf{D}^{+}\right]+\boldsymbol{V}_{a b}^{\mathrm{xc}+}\left[{ }^{q} \mathbf{D}\right] .
\end{aligned}
$$

Here, ${ }^{q} V_{a b}^{\mathrm{xc}+}$ refers to the TRS component of ${ }^{q} V_{a b}^{\mathrm{xc}}$. The TRantisymmetric Fock contribution ${ }^{q} F_{a b}^{-} \in \mathbb{H}_{i \mathbb{R}}^{2 \times 2}$ arises, on the other hand, through the Hartree-Fock exchange and XC potential only and accordingly depends on both ${ }^{q} \mathbf{D}^{+} \in \mathbb{H}_{\mathbb{R}}^{2 n \times 2 n}$ and ${ }^{q} \mathbf{D}^{-} \in \mathbb{H}_{i \mathbb{R}}^{2 n \times 2 n}$,

$$
\begin{aligned}
{ }^{0} F_{a b}^{-} & ={ }^{0} K_{a b}\left[{ }^{q} \mathbf{D}^{-}\right]+{ }^{0} V_{a b}^{\mathrm{xc}-}\left[{ }^{q} \mathbf{D}\right], \\
\boldsymbol{F}_{a b}^{-} & =\boldsymbol{K}_{a b}\left[{ }^{q} \mathbf{D}^{-}\right]+\boldsymbol{V}_{a b}^{\mathrm{xc}-}\left[{ }^{q} \mathbf{D}\right] .
\end{aligned}
$$

Note that the presence of the additional TR-antisymmetric Fock contribution makes the KU 2c/4c SCF calculations, similarly to the non-relativistic $1 \mathrm{c}$ case, twice as expensive as in the $\mathrm{KR}$ regime. ReSpect is currently the only relativistic code that performs $4 \mathrm{c}$ SCF calculations directly in the KU mode and thus includes spinpolarization effects variationally right from the start. To decompose the Hermitian and TR-general KU density matrix $\mathbf{D} \in \mathbb{C}^{4 n \times 4 n}$,

$$
\mathbf{D}=\left(\begin{array}{ll}
\mathbf{D}_{11} & \mathbf{D}_{12} \\
\mathbf{D}_{21} & \mathbf{D}_{22}
\end{array}\right), \quad \mathbf{D}_{11}, \mathbf{D}_{12}, \mathbf{D}_{21}, \mathbf{D}_{22} \in \mathbb{C}^{2 n \times 2 n},
$$

we follow the procedure discussed in Ref. 42 and map D onto the matrix of complex quaternions in agreement with Eq. (37): ${ }^{\mathrm{q}} \mathbf{D} \simeq{ }^{\mathrm{q}} \mathbf{D}^{+}+{ }^{\mathrm{q}} \mathbf{D}^{-} \in \mathbb{H}_{\mathbb{C}}^{2 n \times 2 n}$, where

$$
\begin{aligned}
& { }^{\mathrm{q}} \mathbf{D}^{+}={ }^{0} \mathbf{D}+{ }^{1} \mathbf{D} \mathbf{i}+{ }^{2} \mathbf{D} \mathbf{j}+{ }^{3} \mathbf{D} \mathbf{k}, \\
& { }^{\mathrm{q}} \mathbf{D}^{-}=i\left({ }^{4} \mathbf{D}+{ }^{5} \mathbf{D} \mathbf{i}+{ }^{6} \mathbf{D} \mathbf{j}+{ }^{7} \mathbf{D} \mathbf{k}\right) .
\end{aligned}
$$

The complex quaternion constituents ${ }^{0-7} \mathbf{D} \in \mathbb{R}^{2 n \times 2 n}$ are given by

$$
\begin{array}{rlrl}
{ }^{0} \mathbf{D} & =\mathfrak{R}\left(\mathbf{D}_{1 \mathbf{1 1}}+\mathbf{D}_{22}^{*}\right) / 2, & { }^{4} \mathbf{D}=\mathfrak{I}\left(\mathbf{D}_{11}-\mathbf{D}_{22}^{*}\right) / 2, \\
{ }^{1} \mathbf{D}=\mathfrak{I}\left(\mathbf{D}_{11}+\mathbf{D}_{22}^{*}\right) / 2, & { }^{5} \mathbf{D}=-\mathfrak{R}\left(\mathbf{D}_{11}-\mathbf{D}_{22}^{*}\right) / 2, \\
{ }^{2} \mathbf{D}=\mathfrak{R}\left(\mathbf{D}_{12}-\mathbf{D}_{21}^{*}\right) / 2, & { }^{6} \mathbf{D}=\mathfrak{I}\left(\mathbf{D}_{12}+\mathbf{D}_{21}^{*}\right) / 2, \\
{ }^{3} \mathbf{D}=\mathfrak{I}\left(\mathbf{D}_{\mathbf{1 2}}-\mathbf{D}_{21}^{*}\right) / 2, & { }^{7} \mathbf{D}=-\mathfrak{R}\left(\mathbf{D}_{\mathbf{1 2}}+\mathbf{D}_{21}^{*}\right) / 2,
\end{array}
$$

and because $\mathbf{D}$ is Hermitian, they have the following matrix properties:

$$
{ }^{k} \mathbf{D}={ }^{k} \mathbf{D}^{T}, \quad{ }^{l} \mathbf{D}=-{ }^{l} \mathbf{D}^{T}, \quad k \in 0,5,6,7 ; \quad l \in 1,2,3,4 .
$$


Summary: The SCF module of ReSpect (SCFun) currently supports the following:

- Kramers-restricted and Kramers-unrestricted formalism for 1c (KS), 2c (X2C), and 4c (DKS) Hamiltonians,

- point and finite models for the nuclear charge,

- resolution-of-the-identity integral acceleration for the Coulomb term (RI-J),

- PCM solvent model,

- diagonalization-free SCF approach,

- initial guess based either on a superposition of atomic densities or on a restart from approximate $1 \mathrm{c} / 2 \mathrm{c}$ Hamiltonians, and

- hybrid MPI/OpenMP parallelization.

Readers can find the up-to-date feature list on http://www. respectprogram.org.

\section{A. SCF application}

To demonstrate the performance of the SCFun module in ReSpect, we performed a ground state SCF optimization for trans- $\left[\mathrm{PtCl}_{2}(N \text {-Adam@CB7) })_{2}\right]$, where Adam stands for adamantane and CB7 for cucurbit[7] uril, using the Perdew-Burke-Ernzerhof (PBE) functional, ${ }^{80,81}$ RI-J, ${ }^{42}$ and either the scalar relativistic DKH2 (1c), two-component X2C (2c), or four-component Dirac-Coulomb (4c) Hamiltonian. Uncontracted basis sets of double- $\zeta$ quality were employed: dyall-vdz ${ }^{82,83}$ for Pt and Dunning's cc-pVDZ ${ }^{84}$ for the remaining light elements. In total, the molecular system contained 311 atoms and 6283 scalar Cartesian GTOs, corresponding to 12566 $2 \mathrm{c}$ or $251324 \mathrm{c}$ basis spinors. The structure of the studied system is depicted in Fig. 1.

Figure 2 compares wall-clock times spent in a single SCF iteration on the computationally most intensive SCF steps, namely,

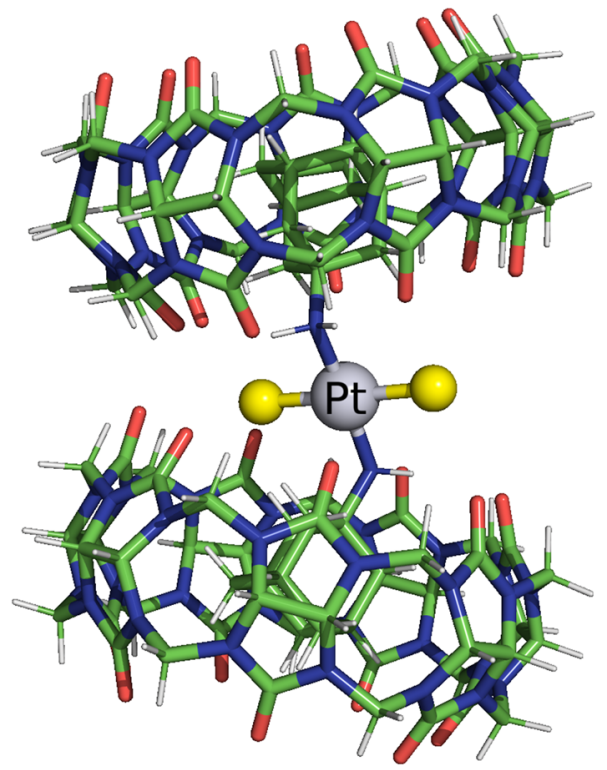

FIG. 1. The molecular structure of trans-[PtCl $\left.2(N-A d a m @ C B 7)_{2}\right]$ used to explore the SCF performance in ReSpect (see Fig. 2).

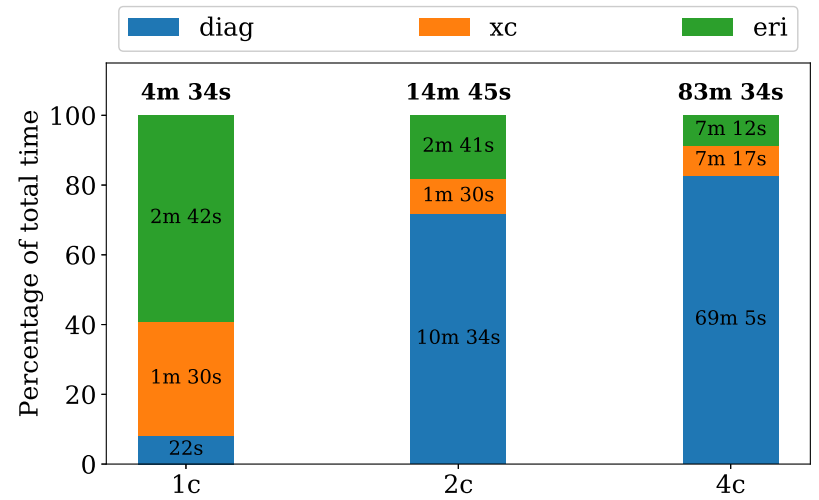

FIG. 2. Wall-clock time per one SCF iteration spent on the matrix diagonalization (diag), and the evaluation of the two-electron Fock (eri) and DFT exchangecorrelation (xc) terms. The OpenMP parallel calculations were performed with the scalar relativistic DKH2 (1c), two-component X2C (2c), and four-component DiracCoulomb (4c) Hamiltonians on a single dual-socket computer node equipped with a $2.50 \mathrm{GHz}$ Intel Xeon E5-2680v3 processor with $24 \mathrm{CPU}$ cores and $128 \mathrm{~GB}$ of memory. The Intel ifort 17.0 compiler with -02 optimization and the parallel Intel MKL library were used for compilation and linking.

the evaluation of the two-electron Fock (eri) and DFT exchangecorrelation (xc) terms and the matrix diagonalization (diag). The wall-clock time for the diagonalization step increases (nearly) cubically with the matrix dimension, as it is expected for a process involving dense matrices. This is the case for the transition from $2 \mathrm{c}$ to $4 \mathrm{c}$, whereas an additional pre-factor of about 4 arises in the step from $1 \mathrm{c}$ to $2 \mathrm{c}$. This pre-factor can be explained by the change of algebra from real (1c) to complex $(2 \mathrm{c})$.

Interestingly, the wall-clock time spent on eri and $x c$ is almost identical for $1 \mathrm{c}$ and $2 \mathrm{c}$. This is because the two-electron Coulomb algorithm can be designed such that all computationally intensive parts are identical for $1 \mathrm{c}$ and $2 \mathrm{c}$. The entire difference in the total computational cost here is thus due to diag, which is the timedetermining step in $2 \mathrm{c}$. In total, the $2 \mathrm{c}$ approach is just a factor of 3.2 more expensive than $1 \mathrm{c}$ for the studied system. On going from $2 \mathrm{c}$ to $4 \mathrm{c}$, the time spent on eri and $\mathrm{xc}$ increases only by a factor of 2.7 and 4.9 , respectively, which is the result of the extra computations associated with the presence of a small-component RKB basis in $4 \mathrm{c}$. The already-dominant diag grows still further in $4 \mathrm{c}$ by a factor of 6.5. However, this is good news for molecular property calculations where the diagonalization step is absent and almost the entire computational cost is that of rebuilding the Fock matrix. Since our interest lies in property calculations, and the 4c Fock construction can cost only as little as 3.4 times more than $1 \mathrm{c}$, we may abandon the (still widespread) opinion of the infeasibility of fully relativistic approaches.

\section{SOLID-STATE SCF}

Systems in the solid state exhibit a discrete translation invariance, i.e., are periodic (and infinite) in one, two, or three spatial dimensions. The Fock operator and the electron density are invariant with respect to translations by a Bravais lattice vector $\boldsymbol{R}{ }^{85}$ Since most properties of solids are determined by the valence 
and conduction-band electrons, the majority of electronic structure codes employ pseudopotentials with plane waves to mitigate the increased computational cost that is inherent in DFT simulations of periodic systems. However, the periodic SCF recently implemented in ReSpect ${ }^{28}$ uses GTOs for all electronic states, treating all KohnSham orbitals on an equal footing. Combining such an all-electron approach with relativistic theories offers several advantages. First, the locality of the real-space GTO basis can be exploited to obtain algorithms whose memory and computational cost increase only linearly with the system size. ${ }^{86,87}$ Second, direct access to all-electron wave functions makes it possible to study core-related properties of solids, such as X-ray spectroscopy and NMR parameters, without the need to use additional partial-wave basis functions (as in the projector-augmented wave method ${ }^{88,89}$ ) to recover the core region. Third, GTOs allow for molecules and solids to be treated within a single theoretical framework, transferring concepts and tools from molecules to the solid-state domain.

\section{A. Periodic SCF theory}

Translation invariance requires a number of modifications to the molecular SCF procedure described in Sec. III. The SCF equation acquires a dependence on the reciprocal lattice vector $\boldsymbol{k} \in \mathcal{K}$ that labels irreducible representations of the translation group. Here, $\mathcal{K}$ denotes the 1st Brillouin zone. Therefore, the matrix form of the SCF equation for periodic systems takes the form

$$
\mathbf{F}(\boldsymbol{k}) \mathbf{C}(\boldsymbol{k})=\mathbf{S}(\boldsymbol{k}) \mathbf{C}(\boldsymbol{k}) \epsilon(\boldsymbol{k}),
$$

where $\mathbf{F}(\boldsymbol{k})$ and $\mathbf{S}(\boldsymbol{k})$ are the Fock and overlap matrices, respectively, $\mathbf{C}(\boldsymbol{k})$ is the $4 \mathrm{c}$ matrix of crystalline orbital (CO) coefficients-the solid-state analog of the MO coefficients [see Eq. (73)] - and $\epsilon(\boldsymbol{k})$ is a diagonal matrix containing the band energies. The Fock and overlap matrices are expressed using the symmetry-adapted $4 \mathrm{c}$ basis,

$$
X_{a}(\boldsymbol{k} ; \boldsymbol{r})=\frac{1}{\sqrt{|\mathcal{K}|}} \sum_{\boldsymbol{R}} e^{i \boldsymbol{k} \cdot \boldsymbol{R}} X_{a}(\boldsymbol{r}-\boldsymbol{R}),
$$

where we replicate and translate the $4 \mathrm{c}$ atom-centered basis functions $X_{a} \equiv X_{a}^{\mathrm{RKB}} \in \mathbb{C}_{+}^{4 \times 4}\left(\mathbb{R}^{3}\right)$ from Eq. (19) to each unit cell located at a lattice site $R$. This construction ensures that both the Bloch condition and the RKB condition are satisfied at the level of the basis. The COs $\psi_{i}(\boldsymbol{k} ; \boldsymbol{r})$ are then expanded in this basis as

$$
\psi_{i}(\boldsymbol{k} ; \boldsymbol{r})=\sum_{a=1}^{n} X_{a}(\boldsymbol{k} ; \boldsymbol{r}) C_{a i}(\boldsymbol{k}),
$$

where $n$ is the number of scalar basis functions in a unit cell. All matrices that enter Eq. (84) are block-diagonal in reciprocal space, i.e., the matrices evaluated for different $\boldsymbol{k}$ points are independent. For each $\boldsymbol{k}$, therefore, solving Eq. (84) proceeds in the same manner as in the molecular case. However, it is sufficient to construct the direct inversion in the iterative subspace (DIIS) error vectors $(\mathbf{e} \equiv[\mathbf{F}, \mathbf{D}])$ only for the $\Gamma(\boldsymbol{k}=\mathbf{0})$ point. ${ }^{87,90}$ The updated real-space density matrix $\mathbf{D}$ is assembled using CO coefficients of all $\boldsymbol{k}$ points as a quadrature

$$
D_{a \boldsymbol{R}, b \mathbf{0}}=\frac{1}{|\mathcal{K}|} \int_{\mathcal{K}} e^{i \boldsymbol{k} \cdot \boldsymbol{R}}\left(\mathbf{C}(\boldsymbol{k}) \mathbf{f}(\boldsymbol{k}) \mathbf{C}^{\dagger}(\boldsymbol{k})\right)_{a b} d^{3} \boldsymbol{k},
$$

where $\mathbf{f}$ is the diagonal matrix of occupation numbers obtained as the zero-temperature limit of the Fermi-Dirac distribution. The integral over $\mathcal{K}$ is evaluated on a uniform mesh of $\boldsymbol{k}$ points with equal weights.

During each SCF cycle, the density matrix is used to compute the Fock operator in real space. Employing the real-space basis functions together with their periodic images $X_{a}(\boldsymbol{r}-\boldsymbol{R})$, one would have to evaluate $N^{2} n^{2}$ matrix elements for each operator, where $N$ is the (formally infinite) number of unit cells. However, translationally invariant operators $\mathbf{A}$ (such as the Fock operator or the overlap matrix) exhibit a Toeplitz structure $A_{a \boldsymbol{R}, b \mathbf{R}^{\prime}}=A_{a \mathbf{0}, b \mathbf{R}^{\prime}-\mathbf{R}}$, which reduces the number of nonequivalent matrix elements to $N n^{2}$. Furthermore, the product of two GTOs $X_{a}^{\dagger}(\boldsymbol{r}) X_{b}(\boldsymbol{r}-\boldsymbol{R})$ decays exponentially with increasing distance between the basis function centers. This enables a very sparse storage of matrix elements, with the number of significant matrix elements increasing only linearly with system size. This data compression is essential to dramatically reduce the memory cost of solid-state simulations in the $4 \mathrm{c}$ periodic SCF module of the ReSpect code. We evaluate the overlap integrals, neglecting the angular part of the basis functions in order to identify and store a list of significant shell pairs before initiating the loop over SCF cycles. Since the reciprocal-space matrices are calculated on-the-fly for each individual $\boldsymbol{k}$ point, our current implementation will only reach the memory limit of standard HPC clusters if one such matrix becomes too large (e.g., for $4 \mathrm{c}$ calculations of unit cells with a very large number of atoms). Therefore, ground-state optimizations of the electronic structure of solids are not more memory demanding than for single molecules.

Periodic SCF in ReSpect operates in the KR regime, i.e., TR symmetry is assumed for all operators. Contributions to the $4 \mathrm{c}$ real-space Fock matrix [kinetic operator, Coulomb interactions, and exchange-correlation (XC) term] are formulated and calculated entirely using quaternion algebra (see Sec. II), ${ }^{28,42,61}$ which significantly reduces the number of computations required at the $4 \mathrm{c}$ level of theory. Combining the translational symmetry with the TR symmetry gives the following structure of the real-space Fock matrix:

$$
F_{a 0, b R}=\left(\begin{array}{cc}
\mathcal{A} & \mathcal{B} \\
-\mathcal{B}^{*} & \mathcal{A}^{*}
\end{array}\right)_{a 0, b \boldsymbol{R}} .
$$

Using the product of two quaternion RKB-basis elements $\Omega_{a \mathbf{0}, b \boldsymbol{R}}(\boldsymbol{r})$ $\equiv{ }^{q} X_{a}^{\dagger}(\boldsymbol{r})^{q} X_{b}(\boldsymbol{r}-\boldsymbol{R}) \in \mathbb{H}_{\mathbb{R}}^{2 \times 2}\left(\mathbb{R}^{3}\right)$, as defined in Eq. (39), we can write the Coulomb term as

$$
J_{a \mathbf{0}, b \boldsymbol{R}}^{\mathrm{e}}=\sum_{\boldsymbol{R}^{\prime}} \int_{\mathbb{R}^{3} \times \mathbb{R}^{3}} \frac{\Omega_{a \mathbf{0}, b \boldsymbol{R}}\left(\boldsymbol{r}_{1}\right) \tilde{\rho}\left(\boldsymbol{r}_{2}\right)}{\left|\boldsymbol{r}_{1}-\boldsymbol{r}_{2}-\boldsymbol{R}^{\prime}\right|} d^{3} \boldsymbol{r}_{1} d^{3} \boldsymbol{r}_{2},
$$

where

$$
\tilde{\rho}(\boldsymbol{r}) \equiv \sum_{\boldsymbol{R}} \operatorname{Tr}\left[\Omega_{a \mathbf{0}, b \boldsymbol{R}}(\boldsymbol{r}) D_{b \boldsymbol{R}, a \mathbf{0}}\right]
$$

is the auxiliary electron density and $\operatorname{Tr}$ denotes the quaternion trace in $\mathbb{H}_{\mathbb{R}}^{2 \times 2}$, which is a special case of the trace defined in Eq. (48). In order to evaluate the infinite long-range lattice sums of electrostatic interactions $\sum_{\boldsymbol{R}^{\prime}}$ in Eq. (89), we employ the multipole expansion of the Coulomb $1 / r_{12}$ operator $^{91}$ and the renormalization procedure applied to the lattice sums of interaction tensor elements. ${ }^{92,93}$ The integration grid used for calculating the XC contribution, as well as 
the construction of the $\mathrm{XC}$ term itself, is adapted to respect the infinite nature of periodic systems according to the scheme of Towler et al. ${ }^{94}$ Further details on our implementation can be found in Ref. 28.

The reciprocal-space Fock matrix is obtained from $F_{a 0, b R}$ as the Fourier series,

$$
F_{a b}(\boldsymbol{k})=\sum_{\boldsymbol{R}} e^{i \boldsymbol{k} \cdot \boldsymbol{R}} F_{a \mathbf{0}, b \boldsymbol{R}}
$$

The TR symmetry of the real-space matrix elements [Eq. (88)] leads to the following matrix structure in reciprocal space:

$$
\mathbf{F}(\boldsymbol{k})=\left(\begin{array}{cc}
\mathcal{A}(\boldsymbol{k}) & \mathcal{B}(\boldsymbol{k}) \\
-\mathcal{B}^{*}(-\boldsymbol{k}) & \mathcal{A}^{*}(-\boldsymbol{k})
\end{array}\right) .
$$

Note that, despite assuming the TR symmetry, the $\boldsymbol{k}$-space Fock matrix formally has a KU structure for each individual $\boldsymbol{k}$ point because, in general, $\mathcal{A}(\boldsymbol{k}) \neq \mathcal{A}(-\boldsymbol{k})$ and $\mathcal{B}(\boldsymbol{k}) \neq \mathcal{B}(-\boldsymbol{k})$. As a consequence, the eigenspectrum of $\mathbf{F}(\boldsymbol{k} \neq 0)$ does not consist of degenerate Kramers partners. The spin splitting due to the lifted degeneracy of $\Gamma$ point, $\bar{\epsilon}(0)=\epsilon(0)$, for an arbitrary $\boldsymbol{k}$ point, $\bar{\epsilon}(\boldsymbol{k}) \neq \epsilon(\boldsymbol{k})$, is known as the Dresselhaus effect ${ }^{95}$ in bulk materials and the Rashba effect $^{96}$ in two-dimensional surfaces and interfaces. Similarly to the $\mathrm{KU}$ case described in Sec. III, the $\boldsymbol{k}$-space Fock matrix is TR-general and requires the use of complex quaternion algebra $\mathbb{H}_{\mathbb{C}}$, ${ }^{28,42}$ i.e., $\mathbf{F}(\boldsymbol{k}) \in \mathbb{H}_{\mathbb{C}}^{2 n \times 2 n}$. Finally note that the TR symmetry of the real-space Fock matrix (88) leads to Kramers partners being each part of a different eigenspectrum, $\mathbf{F}(\boldsymbol{k})$ and $\mathbf{F}(-\boldsymbol{k})$ with $\bar{\epsilon}(\boldsymbol{k})=\epsilon(-\boldsymbol{k})$.

Summary: The periodic SCF module of ReSpect (pSCF) currently supports the following:

- Kramers-restricted formalism for $1 \mathrm{c}(\mathrm{KS})$ and $4 \mathrm{c}$ (DKS) Hamiltonians, ${ }^{28}$

- LDA and GGA XC functionals,

- point and finite models for the nuclear charge,

- all-electron and full-potential setting,

- explicit periodicity for one-, two-, and three-dimensional periodic systems,

- full $\boldsymbol{k}$-point sampling of the Brillouin zone and the use of irreducible unit cells with arbitrary geometries,

- construction of band-structure diagrams for arbitrary paths consisting of line segments in $\boldsymbol{k}$ space and calculation of the density of states,

- initial guess based on a superposition of atomic or molecular densities or on a restart from an approximate 1c Hamiltonian, and

- hybrid MPI/OpenMP parallelization.

Readers can find the up-to-date feature list on http://www. respectprogram.org.

\section{B. User's perspective on periodic SCF}

The periodic SCF ( $\mathrm{pSCF}$ ) module is a standalone library in ReSpect that shares most keywords and features with the molecular SCF. As a result, the Hamiltonian, basis sets, XC integration grid, nucleus model, convergence threshold, and DIIS parameters are specified using the same keywords as for molecules. The geometry block defines the atoms that constitute a unit cell. In addition, users can provide lattice parameters in the lattice block, each line in this block consists of at most three coordinates of one primitive lattice vector that defines a periodic dimension, and the number of $\boldsymbol{k}$ points associated with that dimension. This enables the definition of arbitrary Bravais lattices and unit cell shapes. The total number of periodic dimensions is given by the number of lines in this block, which may range from 0 to 3 . For instance, the lattice parameters of a two-dimensional hexagonal periodic system with a $33 \times 33$ mesh of $\boldsymbol{k}$ points are given as

lattice:

primitive1: $331.23-1.50$

primitive2: 331.231 .50

The initial guess for the density in pSCF can be constructed using a superposition of atomic densities. Alternatively, it is possible to use a converged density matrix from molecular SCF. The default values for the parameters required for the integral approximations (screening and multipole expansion) are chosen to give the best balance between accuracy and performance. However, if needed, these parameters can be adjusted in the input block called peri.

The pSCF solver backs up the density matrix on the hard drive every couple of iterations. This backup can be used to restart the solver from the stored density matrix in case the previous calculation was interrupted before finishing.

To obtain band-structure diagrams of periodic systems, pSCF can calculate band energies $\epsilon_{n}(\boldsymbol{k})$ for arbitrary $\boldsymbol{k}$ points in the final SCF iteration. Any path consisting of line segments in $\boldsymbol{k}$ space can be set up by the user. For some crystal structures, preset paths are available that traverse high-symmetry $\boldsymbol{k}$ points. The program then evaluates and prints the energies for the $\boldsymbol{k}$ points across the given path. Figure 3 illustrates such a diagram for the AgI crystal in the face-centered cubic (fcc) phase together with the density of states evaluated using all band energies. For two-dimensional periodic systems, it is possible to plot the bands $\epsilon_{n}\left(k_{x}, k_{y}\right)$ for all $\left(k_{x}, k_{y}\right) \in \mathcal{K}$. Figure 4 depicts such a surface plot for germanene (a heavier analog of graphene) with a buckled honeycomb structure. Without spinorbit coupling, germanene is a semimetal-the valence and conduction bands touch at a set of isolated (Dirac) $\boldsymbol{k}$ points, forming Dirac cones. Inclusion of spin-orbit coupling, however, opens a bandgap between the cones and turns germanene into a two-dimensional topological insulator.

\section{Example: Silver iodide}

To demonstrate the capabilities of the pSCF module of ReSpect, we calculated the ground state and the band structures of AgI in the fcc crystal phase at the $4 \mathrm{c}$ level of theory. ${ }^{28}$ We employed the uncontracted all-electron double- $\zeta$-quality basis sets of Dyall, dyall-dz. ${ }^{98,99}$ The unit cell contained 255 scalar Cartesian GTOs (92 shells). The basis functions were replicated to form an $11 \times 11$ $\times 11$ supercell, consisting of 2662 heavy atoms in total. The large size of the supercell is due to diffuse functions in the basis set that extend through several unit cells in each direction and thus give a nonnegligible contribution to the overlap integrals. The total number of significant shell pairs in the AgI supercell is 96788 .

Table II shows the memory required to store one $4 \mathrm{c}$ real-space operator (Fock or density matrix) for AgI. Using straightforward complex matrix data structures to store all elements of operators within the given supercell consumes approximately 22 GB of memory. A significant saving is achieved by employing quaternion algebra and by storing only nonzero elements of the quaternion-valued 

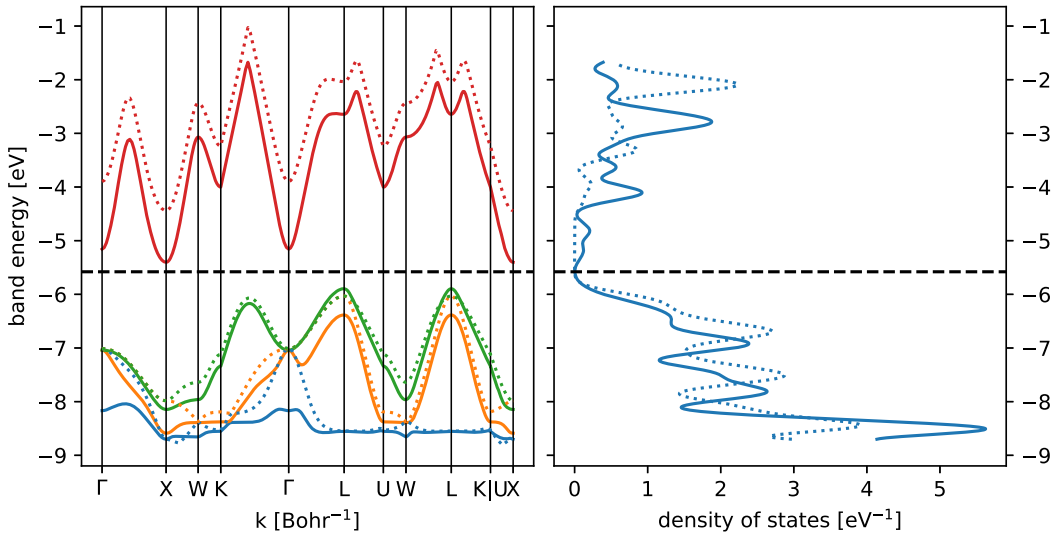

FIG. 3. Band-structure diagram for a path traversing highsymmetry points in reciprocal space (left) and the density of states (right) of Agl. The solid and dashed lines represent the $4 \mathrm{c}$ and $1 \mathrm{c}$ levels of theory, respectively. The horizontal dashed black line separates the occupied and the vacant states. matrix in $\mathbb{H}_{\mathbb{R}}^{2 \times 2}$. Furthermore, the memory is reduced by an additional factor of 138 if only the significant basis-function pairs are stored (see Sec. IV A). The linear scaling of the data structures is crucial for molecular systems containing heavy elements with many basis functions but becomes inevitable when studying periodic systems with large unit cells.

Figure 5 depicts the scaling of the hybrid MPI/OpenMP parallel implementation of the method with respect to the number of central processing units (CPUs) used. Each computational node has the dual-socket 16-core architecture. We utilized 16 OpenMP threads per socket and the Message Passing Interface (MPI) for inter-socket and inter-node communication. The reduction of the total wallclock time (speedup) for both the Coulomb and XC contributions exhibits a near-linear pattern, with a slope very close to the ideal value even when 2048 CPUs (64 nodes) are used. Compared to a similar figure we showed in Ref. 28, the XC term now displays the

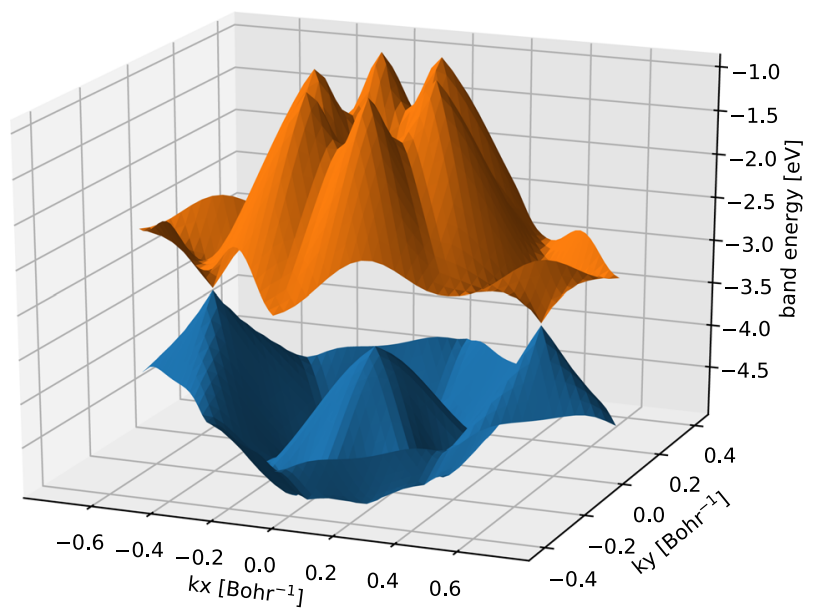

FIG. 4. The highest occupied (blue) and lowest unoccupied (orange) bands of the two-dimensional germanene. Dirac cones can be seen at two distinct Dirac points. A small bandgap opens up between the valence and conduction bands at the Dirac points when spin-orbit coupling is included in the calculation. proper scaling behavior for larger numbers of CPUs. This is due to a minor modification to the $\mathrm{XC}$ routine. Timings for the remaining parts of the algorithm are negligible.

Solid-state calculations employing GTOs are often hampered by numerical instabilities caused by the diffuse functions present in common molecular basis sets, ${ }^{86,100}$ and all-electron basis sets optimized for solid-state calculations are only available for light elements $(Z<36) .{ }^{100}$ Approximations introduced for the evaluation of the Fock matrix elements often break down when very small exponents $(<0.1)$ are used to express the periodic potential of an extended system. These issues can be circumvented by removing the diffuse functions from the basis. ${ }^{100-102}$ However, we found that deleting the smallest exponents in the Ag basis produced errors as large as $1.2 \mathrm{eV}$ for some bandgaps of AgI. ${ }^{28}$ For comparison, the relativistic correction to the bandgap amounts to $1.1 \mathrm{eV}$, and the splitting induced by spin-orbit coupling in high-symmetry $\boldsymbol{k}$ points in the valence band reached $1.13 \mathrm{eV}$. In our earlier implementation, we only experienced issues with diffuse functions in the $4 \mathrm{c}$ scheme, where some of the negative-energy states intruded into the positiveenergy region. It was possible to obtain the proper ground state by identifying the intruder states and leaving them vacant. We recently found that this problem originated in the one-center approximation of the $\left[\Omega^{S S} \mid \Omega^{S S}\right]$ integrals. We have not seen any problems with this approximation when applied to molecules, although a similar issue due to the combination of linear dependency and the one-center approximation for two-electron integrals was noted

TABLE II. Memory requirements (in GB) of various data structure storage options for the $4 \mathrm{c}$ real-space Coulomb and XC operators. The numbers are reported for Agl.

\begin{tabular}{lcc}
\hline \hline \multicolumn{2}{c}{ Storing } & \\
\hline $\begin{array}{l}\text { Scalar basis-function } \\
\text { pairs }\end{array}$ & $\begin{array}{c}\text { Multicomponent } \\
\text { matrix elements }\end{array}$ & Memory (GB) \\
\hline All & All in $\mathbb{C}^{4 \times 4}$ & 22 \\
All & Nonzero in $\mathbb{H}_{\mathbb{R}}^{2 \times 2}$ & 3.5 \\
Significant only & Nonzero in $\mathbb{H}_{\mathbb{R}}^{2 \times 2}$ & 0.025 \\
\hline \hline
\end{tabular}




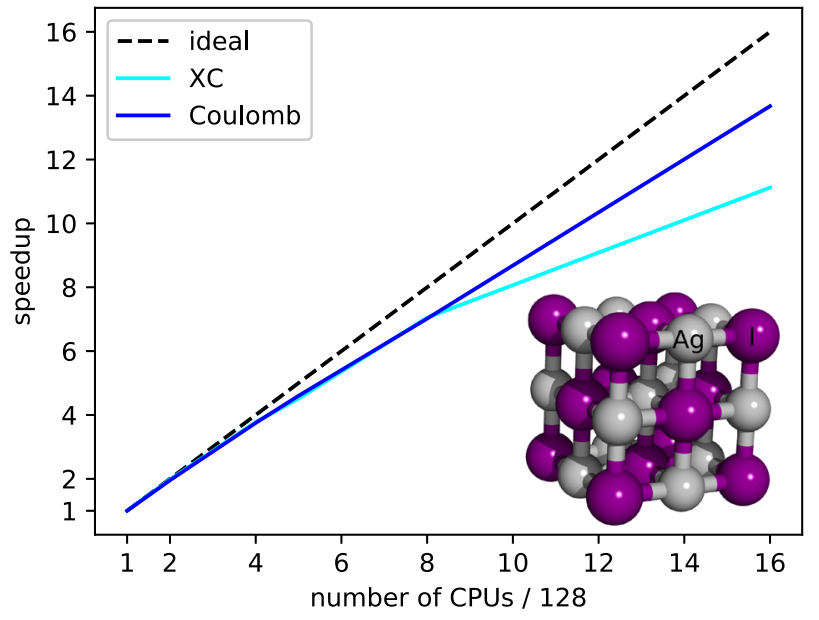

FIG. 5. Reduction of the wall-clock time needed for assembling the real-space Fock matrix terms (Coulomb and XC) for one SCF cycle of the 4c Agl calculation as a function of the number of CPUs used. The computational resources used for this study range from 128 to $2048 \mathrm{CPUs}$ distributed across several nodes, each node containing $32 \mathrm{CPUs}$. The dashed line denotes the ideal linear scaling with the number of computational resources.

for molecules by another study. ${ }^{103}$ Nevertheless, accurate treatment of $\left[\Omega^{S S} \mid \Omega^{S S}\right]$ integrals turned out to be essential for achieving rapid SCF convergence and avoiding the intruder negative-energy states.

\section{ELECTRON PARAMAGNETIC RESONANCE (EPR)}

Electron paramagnetic resonance (EPR) spectroscopy is an important tool in the study of electronic structure of open-shell systems. EPR spectra are usually interpreted in terms of the electronic gtensor, the hyperfine coupling tensor (A-tensor), and the zero-field splitting (ZFS) tensor. Calculations of molecular properties such as the EPR parameters are still a considerable challenge for quantumchemical methods due to the spin-orbit-dominated nature of gtensors and ZFSs and the dependence of A-tensors on spin-density distributions near the nuclei. For EPR parameters, spin-orbit and scalar relativistic effects range from important to crucial, and they increase toward the lower regions of the Periodic Table. In this section, we discuss details of the $4 \mathrm{c}$ DKS calculation of the g-tensor and A-tensor, while the calculation of ZFS energies is described in Sec. X.

In wave-function theories, EPR tensors are obtained by mapping an effective spin Hamiltonian to the quantum-chemical Hamiltonian expressed in the basis of its unperturbed eigenfunctions. In the Kramers-unrestricted DFT formalism, this approach can lead to disastrous results, as discussed in Ref. 104. In the ReSpect program, this is resolved by calculating three Kohn-Sham (KS) determinants with their magnetization oriented along the three Cartesian axes. This is usually achieved by using a scalar-relativistic KS determinant with a well-defined spin oriented in the $x, y$, or $z$ direction as a starting guess for the four-component calculation. The g-tensor and A-tensor are then evaluated as first-order properties ${ }^{29,31}$ (in SI units) as follows:

$$
\begin{aligned}
& g_{u v}=\left.\frac{1}{\mu_{B}\left\langle\widetilde{S}_{v}\right\rangle} \frac{\mathrm{d} E\left(J_{v}\right)}{\mathrm{d} B_{u}}\right|_{B=0}, \\
& A_{u v}^{M}=\left.\frac{1}{h\left\langle\widetilde{S}_{v}\right\rangle} \frac{\mathrm{d} E\left(J_{v}\right)}{\mathrm{d} I_{u}^{M}}\right|_{I^{M}=0},
\end{aligned}
$$

where $\left\langle\widetilde{S}_{v}\right\rangle$ is the effective spin of the system, $h$ is Planck's constant, $\mu_{B}$ denotes the Bohr magneton, $J_{v}$ is the $v$ th component of the magnetization vector, $\boldsymbol{B}$ is the uniform external magnetic field, and $\boldsymbol{I}^{M}$ represents the spin of nucleus $M$. Applying the Hellmann-Feynman theorem, ${ }^{4,105}$ the g- and A-tensors can be expressed in terms of a perturbation-free density matrix $\mathbf{D}\left(J_{v}\right)$ with the magnetization vector oriented along the $v$ direction,

$$
\begin{aligned}
g_{u v} & =\frac{2 c}{\left\langle\widetilde{S}_{v}\right\rangle} \operatorname{Tr}\left[\mathbf{h}^{B_{u}} \mathbf{D}\left(J_{v}\right)\right], \\
A_{u v}^{M} & =\frac{\gamma^{M}}{2 \pi\left\langle\widetilde{S}_{v}\right\rangle} \operatorname{Tr}\left[\mathbf{h}^{\mu_{u}^{M}} \mathbf{D}\left(J_{v}\right)\right],
\end{aligned}
$$

with

$$
\begin{gathered}
h_{\mu \nu}^{B_{u}} \equiv\left\langle\boldsymbol{X}_{\mu}^{(0)}\left|\frac{1}{2}\left(\vec{r}_{G} \times \overrightarrow{\boldsymbol{\alpha}}\right)_{u}\right| \boldsymbol{X}_{v}^{(0)}\right\rangle, \\
h_{\mu \nu}^{\mathrm{pn} / \mathrm{fn}, \mu_{u}^{M}} \equiv\left\langle\boldsymbol{X}_{\mu}^{(0)}\left|\left(\overrightarrow{\boldsymbol{\alpha}} \times \vec{\nabla} \phi_{M}^{\mathrm{pn} / \mathrm{fn}}\right)_{u}\right| \boldsymbol{X}_{v}^{(0)}\right\rangle .
\end{gathered}
$$

Here, $\boldsymbol{\mu}^{M}=\hbar \gamma^{M} \boldsymbol{I}^{M}$, with $\gamma^{M}$ representing the gyromagnetic ratio of nucleus $M$, the RKB basis, $\mathbf{X}^{(0)} \equiv \mathbf{X}^{\mathrm{RKB}}$, as defined in Eq. (18), and $\boldsymbol{r}_{M}$ and $\boldsymbol{r}_{G}$ are the electron position vector, $\boldsymbol{r}$, expressed relative to the position of nucleus $M, \boldsymbol{R}_{M}$, and to the position of the gauge origin, $\boldsymbol{R}_{0}$, respectively. The A-tensor is expressed in terms of either point $(\mathrm{pn})$ or finite $(\mathrm{fn})$ magnetic moment distribution model with functions $\phi_{M}^{\mathrm{pn}}$ and $\phi_{M}^{\mathrm{fn}}$ defined by Eqs. (8) and (9), respectively; and the perturbation-free density matrix, $\mathbf{D}$, is acquired by solving the SCF equation as described in Sec. III.

A useful feature of the ReSpect program for analysis of the gand A-tensor is plotting them as a function of the strength of the spin-orbit interaction or of the speed of light. ${ }^{30,104,106,107}$ To scale the SO effects in the four-component framework, we multiply the ${ }^{0} F^{-}$ and $\boldsymbol{F}^{+}$components of the Fock matrix (77) by a scaling factor, where zero corresponds to the scalar four-component calculation, and one represents the full four-component calculation.

Summary: The calculation of EPR parameters by ReSpect is available for doublet and triplet open-shell molecular systems with the degenerate ground state described by a Kramers-unrestricted KS determinant (paramagnetic systems), and it currently includes the following features:

- 4c (DKS) Hamiltonian,

- noncollinear DFT potentials [(68) and (69)],

- PCM solvent model, ${ }^{45,46}$

- point and finite models for the nuclear charge,

- point and finite models for the nuclear magnetic dipole moment

- MO analysis of EPR tensors, 
- separation of the A-tensor into Fermi-contact, spin-dipolar, paramagnetic spin-orbit, and relativistic contributions according to Ref. 108, and

- spin-orbit analysis, either by scaling SO matrix elements or by using PT2 ${ }^{107}$ and PT3 theories.

Readers can find the up-to-date feature list on http://www. respectprogram.org.

\section{A. EPR applications}

The first 4c DKS implementation of EPR parameters in the ReSpect program was reported in $2010^{29,31}$ and utilized an earlier $2 \mathrm{c}$ approach based on three Kramers-unrestricted self-consistent-field (SCF) DKS determinants with orthogonal orientations of the magnetization vectors. ${ }^{109}$ An initial assessment of the method for smaller heavy-atom containing radicals and for medium-sized molybde$\operatorname{num}(\mathrm{V})$ and tungsten $(\mathrm{V})$ complexes revealed that variational inclusion of relativistic corrections by the $4 \mathrm{c}$ method offers a significant advantage over earlier approaches based on the leading-order perturbation theory. ${ }^{106}$ This can easily be understood by performing a scaling analysis where the EPR parameters are plotted as a function of the strength of the spin-orbit interaction or as a function of the speed of light. The resulting curves were found to be clearly nonlinear for both the g- and A-tensors, and in fact, the analysis confirmed that higher-order spin-orbit (HOSO) contributions may be of the same magnitude as the leading first-order contributions, thus altering the computed EPR tensors fundamentally, in particular for $5 \mathrm{~d}$ species. ${ }^{106}$ For the g-tensors, the HOSO effects were roughly an order of magnitude larger for the $5 \mathrm{~d}$ than for the $4 \mathrm{~d}$ complexes, for instance, amounting to $-180 \mathrm{ppt}(-95 \mathrm{ppt})$ for $\Delta g_{\|}\left(\Delta g_{\perp}\right)$ in the case of $\left[\mathrm{OsOF}_{5}\right.$ ] (see Fig. 6). ${ }^{106}$ Somewhat smaller deviations from linearity were observed for the hyperfine coupling tensors, indicating a smaller HOSO effect overall.

The initial $4 \mathrm{c}$ implementation, however, was restricted to DFT functionals of the local spin density approximation (LSDA) and generalized-gradient approximation (GGA) types, which are known to underestimate the spin density on metal centers. Since it is known that a judicious admixture of Hartree-Fock exchange (HFX) in hybrid functionals can improve both the g-tensors ${ }^{110}$ and the isotropic metal A-tensors of transition-metal complexes, ${ }^{111}$ the ReSpect EPR implementation was therefore extended to hybrid functionals. ${ }^{30}$ For the A-tensors, the main issue is the description of the spin polarization of the metal s-type core-shells (e.g., $2 \mathrm{~s}$ and $3 \mathrm{~s}$ orbitals for $3 \mathrm{~d}$ centers), which is underestimated by (semi)local functionals and enhanced by the HFX admixture. ${ }^{111}$ For g-tensors, the too-covalent metal-ligand bonding at semilocal DFT levels is the main factor that is corrected for by the HFX admixture. In the case of metal-centered spin density, the latter is underestimated at the LSDA or GGA levels, and the admixture of HFX increases the metal spin density in such cases. As the major SO contributions to the g-tensor often arise from the SO coupling associated with the metal, a larger HFX admixture tends to increase the gtensor anisotropies in those cases ${ }^{110}$ (heavy ligand atoms may modify the picture, and ligand-centered radicals behave in an opposite manner ${ }^{112}$ ).

Systematic benchmarking of hybrid functionals and basis sets on a series of 17 small $4 \mathrm{~d}^{1}$ and $5 \mathrm{~d}^{1}$ complexes suggested the computational protocol DKS/PBE0-40HFX ${ }^{80,81,113} /$ dyall-tz $^{82,83,99} /$ iglo-iii $^{114}$ (Hamiltonian/Functional/MetalBasis/LigandBasis), where PBE040HFX refers to the hybrid functional of PBE0 with $40 \%$ HFX. This protocol performed well for both the g- and hyperfine coupling A-tensors, and in general, the need for an appreciable admixture of HFX in hybrid functionals was apparent, particularly for the A-tensor. ${ }^{30}$ As an independent test and an application of the selected computational protocol, larger $\mathrm{Ir}$ (II) and $\mathrm{Pt}$ (III) complexes with $5 d^{7}(S=1 / 2)$ configuration were chosen. Available experimental EPR data reveal unusually large g-tensor anisotropies in these systems. The largest complex in the test set contained 133 atoms (see the structure in Fig. 7), 607 electrons, and more than 3000 scalar GTOs (12000 4c spinor functions) in total. Application of the 4c DFT-based computational protocol confirmed its accuracy for metal complexes with large g-tensor anisotropies and demonstrated the importance of spin-orbit effects beyond the leading order
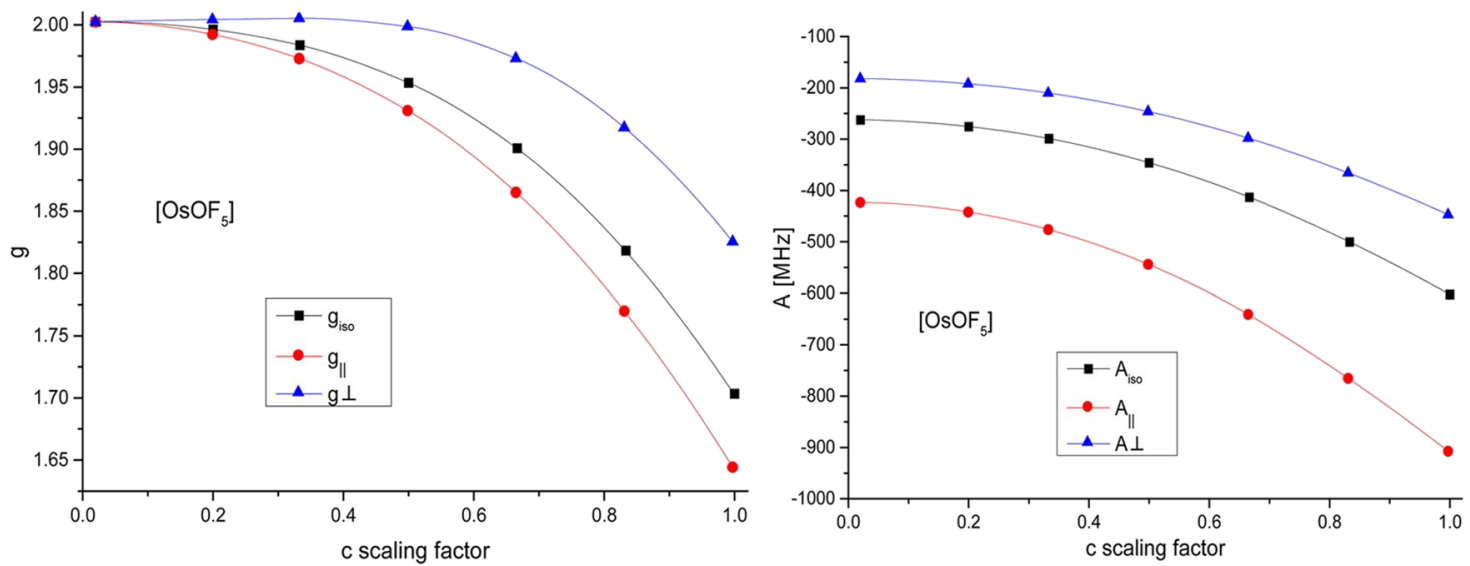

FIG. 6. Analysis of the dependence of $\Delta \mathrm{g}$ - and A-tensor components on the speed of light (and thus on relativistic corrections) for [OsOF $\mathrm{F}_{5}$ ]. Results obtained with the $4 \mathrm{c}$ DKS/PBE0-40HFX/dyall-tz/iglo-iii computational protocol (see text for more details). 


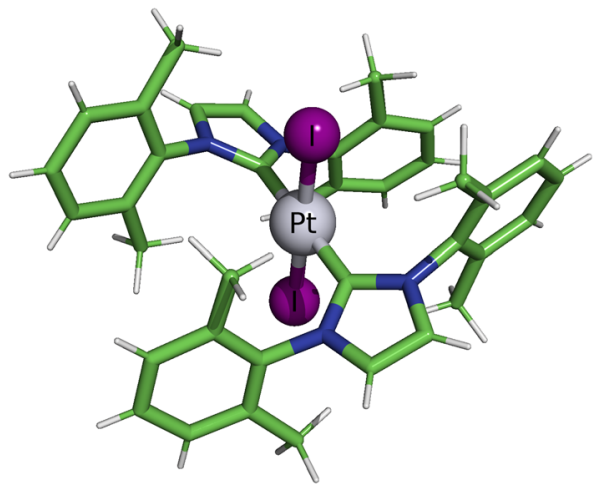

FIG. 7. The molecular structure of $\left[\mathrm{Ptl}_{2}\left(\mathrm{IPr}^{\prime}\right)_{2}\right]^{+}$, a system with an unusually large g-tensor anisotropy. In the IPr' ligand, the isopropyl groups of $\operatorname{IPr}(=1,3-\mathrm{bis}(2,6-$ diisopropylphenyl)imidazole-2-ylidine) were replaced by methyl groups.

in perturbation theory. This holds particularly true for the extreme g-tensor anisotropies, where the higher-order SO effects can easily amount to several hundreds or even thousands of ppt and change the appearance of the g-tensor fundamentally. ${ }^{30}$

\section{NUCLEAR MAGNETIC RESONANCE (NMR)}

Nuclear Magnetic Resonance (NMR) parameters (NMR chemical shifts and indirect nuclear spin-spin coupling constants) provide important information about the electronic structure of systems under study, and NMR is routinely used in many research fields. Calculation of NMR parameters by ab initio methods has become an indispensable tool for analysis and interpretation of experimental data. In the presence of one or several heavy atoms (HA) in the system, an adequate theoretical description of the NMR parameters requires the inclusion of relativistic effects. This holds not only for the prediction of NMR parameters of the HA themselves but also for light atoms, especially, if they are in the vicinity of the HA. For example, the SO effects can shift NMR signals to a completely unexpected range (see Sec. VI A). This can be partially attributed to the fact that magnetic interactions are relativistic in their nature and to the sensitivity of the NMR signals to the electronic structure near the spectator atom.

In this section, we describe the main aspects of the calculation of the NMR shielding tensor, $\sigma^{N}$, and the indirect nuclear spin-spin coupling tensor, $\mathbf{J}^{M N}$, in the framework of four-component DFT theory. ${ }^{32-34}$ In the case of diamagnetic systems, i.e., systems with a non-degenerate ground state and with excited states that are not thermally accessible, the NMR parameters are calculated as a bilinear derivative of the ground state electronic energy (in SI units),

$$
\begin{aligned}
& \sigma_{u v}^{N}=\left.\frac{\mathrm{d}^{2} E}{\mathrm{~d} B_{u} \mathrm{~d} \mu_{v}^{N}}\right|_{B, \mu^{N}=0}, \\
& J_{u v}^{M N}=\left.h \frac{\gamma_{M}}{2 \pi} \frac{\gamma_{N}}{2 \pi} \frac{\mathrm{d}^{2} E}{\mathrm{~d} \mu_{u}^{M} \mathrm{~d} \mu_{v}^{N}}\right|_{\boldsymbol{\mu}^{M}, \boldsymbol{\mu}^{N}=0},
\end{aligned}
$$

where $\boldsymbol{B}$ represents a uniform magnetic field and $\boldsymbol{\mu}^{N}$ and $\boldsymbol{\mu}^{M}$ are the magnetic moments of the nuclei $N$ and $M$, respectively.

In this section, we use complex algebra and employ the fourcomponent flattened atomic orbital basis indices $\mu, v, \lambda$, and $\tau$ (for more details, see Ref. 39), and bold font indicates either a vector or matrix quantity, depending on the context. Furthermore, we use the following index conventions: $i$ and $j$ represent occupied MO orbitals, $a$ and $b$ denote unoccupied positive- and negativeenergy MO orbitals, respectively, and $p$ and $q$ are general MO orbitals.

The bilinear derivatives of the energy in Eqs. (99) and (100) may be expressed as a sum of diamagnetic and paramagnetic contributions,

$$
\left.\frac{\mathrm{d}^{2} E}{\mathrm{~d} \zeta_{u} \mathrm{~d} \mu_{v}^{N}}\right|_{\zeta, \boldsymbol{\mu}^{N}=0}=\operatorname{Tr}\left[\mathbf{h}^{(u, v)} \mathbf{D}^{(0,0)}\right]+\operatorname{Tr}\left[\mathbf{h}^{(0, v)} \mathbf{D}^{(u, 0)}\right]
$$

In the following discussion, setting the parameter $\boldsymbol{\zeta}$ to $\boldsymbol{B}$ or $\boldsymbol{\mu}^{M}$ will provide the expressions for the NMR shielding tensor or the indirect nuclear spin-spin coupling tensor, respectively. The matrix $\mathbf{h}^{(0, v)}$ has the same form for both properties (99) and (100),

$$
\mathbf{h}^{(0, v)}=\mathbf{h}^{\mathrm{pn}, \mu_{v}^{N}} \text {, }
$$

where the matrix $\mathbf{h}^{\mathrm{pn}, \mu_{v}^{N}}$ is defined in Eq. (98). In order to achieve faster convergence with respect to the basis in the four-component relativistic framework, it is necessary to use the RMB basis for the description of the small component of the molecular orbitals. Furthermore, in the case of the NMR shielding calculations, the socalled gauge-including atomic orbitals must be employed along with the RMB condition, as discussed in Sec. II C. The use of the RMB or RMB-GIAO allows one to obtain the explicit diamagnetic contribution [the first term on the RHS of Eq. (101)]. ${ }^{32}$ If neither of these conditions is fulfilled, four-component theories of the magnetic properties yield paramagnetic terms only. The bilinear matrix $\mathbf{h}^{(u, v)}$ can then be written as

$$
h_{\mu v}^{(u, v)}=\left\langle\boldsymbol{X}_{\mu}^{(0)}\left|\left(\overrightarrow{\boldsymbol{\alpha}} \times \vec{\nabla} \phi_{N}^{\mathrm{pn}}\right)_{v}\right| \boldsymbol{X}_{v}^{\zeta_{u}}\right\rangle+\text { h.c. }
$$

Here, the function $\phi_{N}^{\mathrm{pn}}$ is defined in Eq. (8), the RKB basis $\mathbf{X}^{(0)} \equiv \mathbf{X}^{\mathrm{RKB}}$ is defined in Eq. (18), and the basis induced by the magnetic field $\boldsymbol{X}_{v}^{\zeta_{u}}$ can be constructed from the basis spinors $\boldsymbol{X}_{v}^{\mathrm{RMB}}(\boldsymbol{r}, \Lambda, \boldsymbol{A})$ [Eqs. (32) and (33)] using the corresponding gauge function and vector potential,

$$
\begin{gathered}
\boldsymbol{X}_{v}^{B_{u}} \equiv \boldsymbol{X}_{v}^{\mathrm{RMB}-\mathrm{GIAO}, B_{u}}=\frac{\mathrm{d}}{\mathrm{d} B_{u}}\left(\boldsymbol{X}_{v}^{\mathrm{RMB}}\right), \\
\boldsymbol{X}_{v}^{\mu_{u}^{M}} \equiv \boldsymbol{X}_{v}^{\mathrm{RMB}, \mu_{u}^{M}}=\frac{\mathrm{d}}{\mathrm{d} \mu_{u}^{M}}\left(\boldsymbol{X}_{v}^{\mathrm{RMB}}\right), \\
\boldsymbol{A}=\frac{1}{2}\left(\boldsymbol{B} \times \boldsymbol{r}_{G}\right), \quad \Lambda_{v}=[\text { Eq. }(27)], \\
\boldsymbol{A}=\nabla \phi_{M}^{\mathrm{pn}} \times \boldsymbol{\mu}^{M}, \quad \Lambda_{v}=0 .
\end{gathered}
$$


The perturbation-free and response density matrices have the form

$$
\begin{aligned}
\mathbf{D}^{(0,0)} & \equiv \mathbf{D}^{(0)}=\mathbf{C f}^{\mathrm{o}} \mathbf{C}^{\dagger}, \\
\mathbf{D}^{(u, 0)} & \equiv \mathbf{D}^{\mathrm{o}, u}+\mathbf{D}^{\mathrm{v}, u}, \\
\mathbf{D}^{\mathrm{o}, u} & =-\mathbf{D}^{(0)}\left\langle\boldsymbol{\Omega}^{\zeta_{u}}\right\rangle \mathbf{D}^{(0)}, \\
\mathbf{D}^{\mathrm{v}, u} & =\mathbf{C f}^{\mathrm{v}} \boldsymbol{\beta}^{\zeta_{u}} \mathbf{f}^{\mathrm{o}} \mathbf{C}^{\dagger}+\text { h.c. },
\end{aligned}
$$

with the selection matrices and the RMB overlap distribution defined as

$$
\begin{gathered}
f_{p q}^{o}=\delta_{p i} \delta_{i q}, \quad f_{p q}^{\mathrm{v}}=\delta_{p a} \delta_{a q}, \\
\boldsymbol{\Omega}^{\zeta_{u}}=\widetilde{\boldsymbol{\Omega}}^{\zeta_{u}}+\left(\widetilde{\boldsymbol{\Omega}}^{\zeta_{u}}\right)^{\dagger}, \quad \widetilde{\boldsymbol{\Omega}}_{\mu \nu}^{\zeta_{u}}=\left(\boldsymbol{X}_{\mu}^{(0)}\right)^{\dagger} \boldsymbol{X}_{v}^{\zeta_{u}} .
\end{gathered}
$$

The perturbation-free MO coefficients, $\mathbf{C}$, and one-electron energies, $\varepsilon_{p}$, are solutions of the eigenvalue equation (73). Although it is possible to solve the response equations in the $\mathrm{AO}$ basis, ${ }^{115,116}$ in practice this is rarely done. The best convergence is obtained by MObased solvers, i.e., the best preconditioning of the residuum vectors is known in the MO basis. Another advantage of MO-based solvers is that it becomes necessary only to search for the vacant-occupied block of the response density matrix, $\beta_{a i}$. The linear response equations for the NMR shielding and indirect nuclear spin-spin coupling tensor correspond to the damped response TDDFT equation [Eq. (137), vide infra] when $\omega=0, \gamma=0, \mathbf{X}=\boldsymbol{\beta}$, and $\mathbf{Y}=\boldsymbol{\beta}^{*}$, and the RHS of the equation contains both the magnetic moment operator and contributions from the RMB basis. Due to these constraints on Eq. (137), the problem can be reduced to

$$
\mathbf{A} \boldsymbol{\beta}^{\zeta_{u}}+\mathbf{B}\left(\boldsymbol{\beta}^{\zeta_{u}}\right)^{*}=-\mathbf{P}^{\zeta_{u}},
$$

where the matrices $\mathbf{A}$ and $\mathbf{B}$ have the form

$$
\begin{aligned}
A_{a i, b j} & =\left(\varepsilon_{a}-\varepsilon_{i}\right) \delta_{a b} \delta_{i j}+K_{\mu v, \lambda \tau} C_{\mu a}^{*} C_{v i} C_{\lambda j}^{*} C_{\tau b}, \\
B_{a i, b j} & =K_{\mu v, \lambda \tau} C_{\mu a}^{*} C_{v i} C_{\lambda b}^{*} C_{\tau j} .
\end{aligned}
$$

The perturbation matrix $\mathbf{P}$ can be written as

$$
\begin{aligned}
P_{a i}^{\zeta_{u}} \equiv & P_{\mu \nu}^{\zeta_{u}} C_{\mu a}^{*} C_{v i}, \\
P_{\mu \nu}^{B_{u}}= & h_{\mu \nu}^{B_{u}}-\varepsilon_{i}\left\langle\Omega_{\mu \nu}^{B_{u}}\right\rangle+K_{\mu v, \lambda \tau}(\xi) D_{\tau \lambda}^{\mathrm{o}, u}+\left\langle\boldsymbol{X}_{\mu}^{(0)}\left|\mathbf{h}^{\mathrm{D}}\right| \boldsymbol{X}_{v}^{B_{u}}\right\rangle+\text { h.c. } \\
& +V^{\mathrm{xc}}\left(\Omega_{\mu \nu}^{B_{u}}, \xi\right)+K^{\mathrm{xc}}\left(\Omega_{\mu \nu}^{(0)}, \Omega_{\lambda \tau}^{B_{u}}, \xi\right) D_{\tau \lambda}^{(0)} \\
& +\left\{\left[\Omega_{\mu \nu}^{(0)} \mid \Omega_{\lambda \tau}^{B_{u}}\right]+\left[\Omega_{\mu \nu}^{B_{u}} \mid \Omega_{\lambda \tau}^{(0)}\right]\right\} D_{\tau \lambda}^{(0)} \\
& -\xi\left\{\left[\Omega_{\mu \lambda}^{(0)}\left|D_{\lambda \tau}^{(0)}\right| \Omega_{\tau \nu}^{B_{u}}\right]+\left[\Omega_{\mu \lambda}^{B_{u}}\left|D_{\lambda \tau}^{(0)}\right| \Omega_{\tau \nu}^{(0)}\right]\right\} \\
P_{\mu \nu}^{\mu_{u}^{M}}= & h_{\mu \nu}^{\mathrm{pn}, \mu_{u}^{M}}+\left(\varepsilon_{a}-\varepsilon_{i}\right)\left|\widetilde{\Omega}_{\mu \nu}^{\mu_{u}^{M}}\right\rangle+K_{\mu \nu, \lambda \tau}(\xi) D_{\tau \lambda}^{\mathrm{o}, u} \\
& +\left\{K^{\mathrm{xc}}\left(\Omega_{\mu \nu}^{(0)}, \Omega_{\lambda \tau}^{\mu_{u}^{M}}, \xi\right)+\left[\Omega_{\mu \nu}^{(0)} \mid \Omega_{\lambda \tau}^{\mu_{u}^{M}}\right]\right\} D_{\tau \lambda}^{(0)} \\
& -\xi\left\{\left[\Omega_{\mu \lambda}^{(0)}\left|D_{\lambda \tau}^{(0)}\right|\left(\widetilde{\Omega}^{\mu_{u}^{M}}\right)_{\nu \tau}^{*}\right]+\left[\widetilde{\Omega}_{\mu \lambda}^{\mu_{u}^{M}}\left|D_{\lambda \tau}^{(0)}\right| \Omega_{\tau \nu}^{(0)}\right]\right\},
\end{aligned}
$$

where

$$
\begin{gathered}
V^{\mathrm{xc}}\left(\Omega_{\mu v}, \xi\right) \equiv V_{\mu \nu}^{\mathrm{xc}}(\xi), \\
K^{\mathrm{xc}}\left(\Omega_{\mu v}, \Omega_{\lambda \tau}, \xi\right) \equiv K_{\mu v, \lambda \tau}^{\mathrm{xc}}(\xi),
\end{gathered}
$$

$K_{\mu \nu, \lambda \tau}(\xi) \equiv\left[\Omega_{\mu \nu}^{(0)} \mid \Omega_{\lambda \tau}^{(0)}\right]-\xi\left[\Omega_{\mu \lambda}^{(0)} \mid \Omega_{\tau \nu}^{(0)}\right]+K^{\mathrm{xc}}\left(\Omega_{\mu \nu}^{(0)}, \Omega_{\lambda \tau}^{(0)}, \xi\right)$,

with both $V_{\mu \nu}^{\mathrm{xc}}$ and $K_{\mu \nu, \lambda \tau}^{\mathrm{xc}}$ as defined in Sec. II E, while the matrices $\mathbf{h}^{B_{u}}$ and $\mathbf{h}^{D}$ are described in Eq. (97) and Sec. II A, respectively. The construction of the two-electron repulsion integrals, $[\Omega \mid \Omega]$, is discussed in Sec. II D with the final expression in Eq. (44) written in the complex quaternion algebra. To obtain ERIs in the complex algebra used in this section, it is necessary to use the algebraic isomorphism described in Eqs. (21) and (34).

The difference in the formulation of the response equation [Eq. (114)] for NMR shielding and indirect nuclear spin-spin coupling tensors lies in the choice of the variational parameters,

$$
\begin{gathered}
\left.C_{\mu a}^{*} \frac{\mathrm{d}}{\mathrm{d} B_{u}}\left(\frac{\mathrm{d} L}{\mathrm{~d} C_{\mu i}^{*}}\right)\right|_{B=0}=0, \\
\left\langle\varphi_{a}\left|\frac{\mathrm{d}}{\mathrm{d} \mu_{u}^{M}}\left(\frac{\delta L}{\delta \varphi_{i}^{\dagger}}\right)\right|_{\mu^{M}=0}=0,\right.
\end{gathered}
$$

with the Lagrangian defined as

$$
L=E-\varepsilon_{i}\left(\left\langle\varphi_{i} \mid \varphi_{i}\right\rangle-1\right) .
$$

First, note that the difference between these approaches is due to the magnetically dependent basis set. Therefore, when using basis sets that have no dependence on the perturbation, employing MO coefficients or MOs as variational parameters results in the same working equations. On the other hand, when the basis depends on the perturbation, using MO coefficients as variational parameters leads to methods with a faster basis set convergence. However, because the RMB basis for the magnetic moment of the nucleus ${ }^{34}$ is of order $c^{-2}$, the convergence with the basis is acceptable, and triple-zeta quality basis sets usually give satisfactory results. ${ }^{117}$ The main motivation for using a potentially inferior formulation for the calculation of indirect nuclear spin-spin coupling tensors is to decrease the importance of the two-electron Coulomb integrals for the final results, since these are neglected in our current implementation [the last three terms in Eq. (118)]. Note that in the case of non-hybrid DFT calculations of closed-shell systems, there is no approximation involved, since these integrals do not contribute to the final results.

The expressions in Eqs. (101)-(121) are valid, regardless of the nature of the reference Kohn-Sham (KS) determinant (Kramersrestricted or Kramers-unrestricted), and they can thus be applied for the calculation of NMR parameters of closed-shell (diamagnetic) systems as well as for the calculation of the orbital term of the open-shell (paramagnetic) systems (see Sec. VII). However, in the case of the closed-shell reference KS determinant, the two-electron terms in the response equation [Eq. (114)] simplify considerably, since the trace of the matrix product of the Hermitian time-reversal symmetric and the Hermitian time-reversal anti-symmetric matrix is zero. Thus, for example, the Coulomb integral $\left[\Omega_{\mu \nu}^{(0)} \mid \Omega_{\lambda \tau}^{B_{u}}\right] D_{\tau \lambda}^{(0)}$ vanishes. 
Summary: The calculation of NMR parameters by ReSpect is available for closed-shell molecular systems with a non-degenerate ground state described by a Kramers-restricted KS determinant (diamagnetic systems), and it currently includes the following features:

- 4c (DKS) Hamiltonian,

- noncollinear DFT potentials and kernels as listed in Table III,

- resolution-of-the-identity integral acceleration for the Coulomb term (RI-J),

- PCM solvent model for both perturbation-free $e^{45,46}$ and response MOs,

- analysis of spin-orbit and scalar relativistic effects by turning off SO interactions and by increasing the speed of light (a factor of 10 is usually sufficient to reach the nonrelativistic limit),

- point and finite models for the nuclear charge,

- point and finite models for the nuclear magnetic dipole moment and nuclear spin-spin couplings,

- MO analysis of the paramagnetic NMR contributions,

- MO analysis of the spin-orbit contributions to the NMR shielding tensor using PT3, ${ }^{118,119}$ and

- hybrid MPI/OpenMP parallelization.

Note that the feature list related to NMR for paramagnetic systems (pNMR) is listed in Sec. VII. Readers can find the up-to-date feature list on http://www.respectprogram.org.

\section{A. NMR applications}

One of the areas where applications of ReSpect have had the largest impact so far is in the 4c relativistic computation of NMR parameters for heavy-atom systems. The initial 4c DKS implementation of NMR in ReSpect, which introduced a new theoretical RMB-GIAO concept, ${ }^{32-34}$ was successfully assessed on the ${ }^{1} \mathrm{H}$ shift

TABLE III. Expressions used by the NMR module for the calculation of the noncollinear exchange-correlation potential and kernel. $^{\text {a }}$

\begin{tabular}{lcccc}
\hline \hline Reference KS determinant & Method & $E^{\mathrm{xc}}$ & $\boldsymbol{V}^{\mathrm{xc}}$ & $\boldsymbol{K}^{\mathrm{xc}}$ \\
\hline \multirow{3}{*}{ Closed-shell } & GGA $^{\mathrm{b}}$ & $(58)$ & $(67)$ & $(70)^{\mathrm{c}}$ \\
& GGA $^{\mathrm{d}}$ & $(58)$ & $(67)$ & $(71)$ \\
& ALDA & $(58)$ & $(67)$ & $(72)^{\mathrm{c}}$ \\
\hline \multirow{3}{*}{ Open-shell } & GGA $^{\mathrm{b}}$ & $(66)$ & $(69)$ & $\mathrm{e}$ \\
& ALDA $^{\mathrm{d}}$ & $(66)$ & $(69)$ & $\mathrm{f}$ \\
& ALDA $^{\mathrm{d}}$ & $(63)$ & $(68)$ & $\mathrm{f}$ \\
\hline \hline
\end{tabular}

${ }^{\mathrm{a}}$ The numbers in parentheses indicate the corresponding equations in this article.

${ }^{\mathrm{b}}$ Recommended option.

${ }^{c}$ For closed-shell systems, the magnetically induced charge density and its gradient are zero, and there are, therefore, no contributions from terms containing these quantities.

${ }^{\mathrm{d}}$ This option has been used up to now in all applications of the ReSpect program, where the analytical evaluation of the kernel was used.

${ }^{\mathrm{e}}$ The noncollinear xc kernel as defined by Table I in Ref. 39.

${ }^{\mathrm{f}}$ The noncollinear xc kernel as defined by Eq. (25) in Ref. 39, neglecting terms containing the gradients of the charge and spin density. predictions in transition-metal hydride complexes with large spinorbit (SO) effects. ${ }^{120}$ While the importance of SO effects for the large positive ${ }^{1} \mathrm{H}$ shifts of $\mathrm{d}^{10}$ mercury hydride complexes had already been shown in perturbational SO treatments, ${ }^{121,122}$ the occasionally large negative shifts in heavy $\mathrm{d}^{6}$ and $\mathrm{d}^{8}$ complexes were assumed to arise only partly from SO effects. A clear-cut confirmation was obtained from 4c ReSpect calculations in Ref. 120, which were done with the generalized-gradient approximation (GGA) type of functionals as hybrid functionals had been implemented in ReSpect's NMR property module a bit later. ${ }^{36,123}$ Up to $66 \%$ of the record negative ${ }^{1} \mathrm{H}$ shifts (from -50 to $-60 \mathrm{ppm}$ ) in certain iridium hydride complexes were shown to be due to SO effects, whereas about one third of the large shielding was assigned to the Buckingham-Stephens effect (paramagnetic shielding contributions due to off-center ring-current effects). ${ }^{124}$ These observations subsequently led to a systematic evaluation of trans-ligand influences (TLI) on the ${ }^{1} \mathrm{H}$ shifts in a series of platinum(II) hydride complexes, ${ }^{125}$ and ultimately to an even more general appraisal of TLI for $5 \mathrm{~d}^{6}, 5 \mathrm{~d}^{8}$, and $5 \mathrm{~d}^{10}$ complexes, where sometimes even the sign of the SO effects could change with the choice of the trans ligand. ${ }^{118,126}$ The most detailed interpretations of the sign and magnitude of such SO effects on neighbor-atom NMR shifts were obtained with a third-order perturbation analysis implemented in ReSpect, ${ }^{118}$ allowing the interpretation of various trends in SO-induced shieldings over the Periodic Table. ${ }^{119}$ A recent comprehensive review of relativistic neighbor-atom effects on NMR chemical shifts ${ }^{127}$ emphasized the same third-order perturbation scheme but also used 4c ReSpect shielding calculations to illustrate the most important mechanisms, augmented by plots of real-space functions such as the spin-orbit electron deformation density (SO-EDD), ${ }^{118}$ the SOand-magnetically induced spin density (SOM-ISD), ${ }^{119}$ and induced current densities. $^{128}$

The work of Hrobarik et al. on uranium complexes ${ }^{129}$ that predicted the record positive ${ }^{1} \mathrm{H}$ shifts of more than $+100 \mathrm{ppm}$ for uranium(VI) hydride complexes, and the related large ${ }^{13} \mathrm{C}$ shifts in organouranium(VI) complexes, highlighted large discrepancies between 4c ReSpect and 2c ZORA ADF computations that have also been observed elsewhere. ${ }^{130,131}$ Whereas the ZORA results were used in the initial work, it was subsequently found that the ADF $2 \mathrm{c}$ ZORA implementation had been lacking the exchange-correlation kernel, thus explaining the observed discrepancies. Using a modified ADF implementation with an approximate kernel gave much better agreement between the 2c ZORA and 4c ReSpect DKS shift calculations for both of the above-mentioned studies of TLI, confirming the correctness of the kernel in ReSpect and correcting the preferred exact-exchange admixture to be used in hybrid functionals to predict such SO-induced shifts when the XC kernel is present. $^{12}$

Four-component NMR chemical shift computations with ReSpect have also been used to predict unusual ${ }^{13} \mathrm{C}$ and ${ }^{29} \mathrm{Si} \mathrm{NMR}$ signals of ligand atoms directly bonded to $\mathrm{Tl}(\mathrm{I})$ or $\mathrm{Pb}$ (II) heavyelement centers, which resonate at very high frequencies, up to $400 \mathrm{ppm}$ for ${ }^{13} \mathrm{C}$ and over $1000 \mathrm{ppm}$ for ${ }^{29} \mathrm{Si}$, outside the typical experimental ranges for those nuclei. These unusual chemical shifts have been ascribed to sizable relativistic SO effects, which can amount to more than $200 \mathrm{ppm}$ for ${ }^{13} \mathrm{C}$ and more than 1000 ppm for ${ }^{29} \mathrm{Si}$, values unexpected for diamagnetic compounds of main-group elements. ${ }^{12}$ Interestingly, the theoretical prediction of 
+30.8 ppm for the ${ }^{1} \mathrm{H}$ shift in a $\mathrm{Pb}$ (II) hydride (Fig. 8) ${ }^{132}$ led to the experimental preparation and characterization of a longsought lead(II) hydride complex with a world record ${ }^{1} \mathrm{H}$ chemical shift of $+31.4 \mathrm{ppm}^{133}$ In this case, the SO effect accounts for about $+26 \mathrm{ppm}$ and thus lifts the ${ }^{1} \mathrm{H}$ shift outside the experimentally established chemical shift range of +20 to $-60 \mathrm{ppm}$ for heavy-metal hydrides, which is why the system had remained undiscovered for many years. The theoretical prediction was based on the $\mathrm{DKS} / \mathrm{PBE}^{80,81} /$ dyall-vtz $^{98} /$ dyall-vdz $^{98}$ computational protocol (Hamiltonian/Functional/MetalBasis/LigandBasis), and the studied system contained 102 atoms and more than 2800 scalar GTOs (11 $2004 \mathrm{c}$ spinor functions).

Beyond predicting new ranges of NMR shielding and aiding the interpretation of SO-induced NMR shifts in various heavy elementscontaining systems, $4 \mathrm{c}$ chemical shielding calculations with ReSpect have also been used successfully for determining new absolute shielding scales of heavier nuclei. ${ }^{43,134-137}$ It was observed that the disagreement between experimental determinations and $4 \mathrm{c}$ relativistic calculations of the absolute shielding constants of heavy nuclei is due to the breakdown of the commonly assumed Flygare relation ${ }^{138}$ between the electronic contribution to the nuclear spin-rotation constants and the paramagnetic contribution to the NMR shielding constants. ${ }^{43}$ The implementation of the relativistic method for the calculation of the nuclear spin-rotation constants within the ReSpect program was presented in Ref. 44 and followed closely the theoretical works. ${ }^{139-141}$ Since the absolute chemical shielding constants are important for determining the nuclear magnetic dipole moments, the breakdown of the Flygare relation that results in an error of about $1000 \mathrm{ppm}$ for ${ }^{119} \mathrm{Sn}^{43}$ has significant consequences and has called for the revision of many nuclear magnetic dipole moments. ${ }^{142}$

Finally, we would like to note that the $4 \mathrm{c}$ nuclear spin-spin coupling implementation in ReSpect is as efficient as the code for NMR shieldings [for example, see Ref. 117 with a platinum(II) system containing 110 atoms]. However, the implementation was limited so far to closed-shell diamagnetic systems. ${ }^{143-145}$ This limitation has now been lifted, and Sec. VII reports the first 4c nuclear spin-spin coupling results for paramagnetic systems.

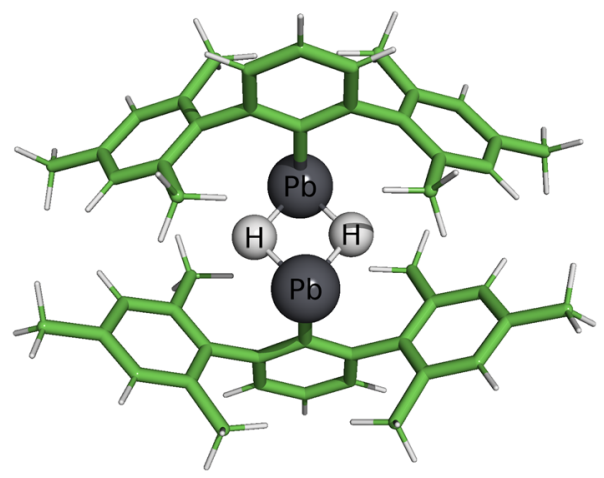

FIG. 8. The molecular structure of $\left[\mathrm{ArPb}(\mu-\mathrm{H})_{2} \mathrm{PbAr}\right]$, which holds the world record for the experimental ${ }^{1} \mathrm{H}$ chemical shift. $\mathrm{Ar}=\mathrm{C}_{6} \mathrm{H}_{3}-2,6-\mathrm{Mes}_{2}$, Mes $=\mathrm{C}_{6} \mathrm{H}_{2}-2,4$, 6-Me $\mathrm{Me}_{3}$. The experimental ${ }^{1} \mathrm{H}$ shift of $+31.4 \mathrm{ppm}^{133}$ agrees well with the ReSpect prediction of $+30.8 \mathrm{ppm}$ of which $+26.0 \mathrm{ppm}$ is due to the spin-orbit interaction.

\section{NUCLEAR MAGNETIC RESONANCE FOR PARAMAGNETIC SYSTEMS (pNMR)}

NMR spectroscopy of open-shell (paramagnetic) species provides significant challenges from both a theoretical and an experimental point of view. Fast nuclear spin relaxation may result in significant broadening of the NMR peaks, which decreases the useful information that can be extracted from the spectrum. On the other hand, the expansion of the spectral window by hundreds (for ${ }^{1} \mathrm{H}$ ) or even thousands (for ${ }^{13} \mathrm{C}$ or ${ }^{15} \mathrm{~N}$ ) of ppm, and the temperature dependence of signals, increases the complexity of the spectra and thus the possible amount of accessible information. From the theoretical point of view, the often multi-configurational nature of the paramagnetic systems, and the importance of core atomic regions for the proper description of the spin polarization effects, makes post-Hartree-Fock methods unsuitable for the full description of paramagnetic NMR (pNMR) spectra. Although DFT is more successful in describing spin polarization than post-Hartree-Fock methods, its single-determinant nature limits its area of application. For these reasons, it is today common to predict paramagnetic NMR spectra either combining post-Hartree-Fock and DFT methods or using DFT methods alone for systems in which the ground state is well described by a single-determinant. The latter is possible mostly for systems with a doubly degenerate ground state (with one unpaired electron) or in cases where low-spin triplet states can be sufficiently described by orienting the magnetization of a single Kohn-Sham (KS) determinant. An added advantage when using DFT-only methodologies, such as used in the ReSpect program, is their internal consistency.

A distinct feature of open-shell systems is that at thermal equilibrium, there is more than one thermally accessible state. In this case, it is necessary to perform a thermal average of the energy when calculating NMR parameters. The NMR shielding tensor and indirect nuclear spin-spin coupling tensors are then defined as (in SI units)

$$
\begin{aligned}
& \sigma_{u v}^{N}=\left.\frac{\mathrm{d}^{2}\langle E\rangle}{\mathrm{d} B_{u} \mathrm{~d} \mu_{v}^{N}}\right|_{\boldsymbol{B}, \boldsymbol{\mu}^{N}=0}, \\
& J_{u v}^{M N}=\left.h \frac{\gamma_{M}}{2 \pi} \frac{\gamma_{N}}{2 \pi} \frac{\mathrm{d}^{2}\langle E\rangle}{\mathrm{d} \mu_{u}^{M} \mathrm{~d} \mu_{v}^{N}}\right|_{\boldsymbol{\mu}^{M}, \boldsymbol{\mu}^{N}=0} .
\end{aligned}
$$

Thermally accessible states include Zeeman-split ground states, zero-field split (ZFS) states, or other low-lying excited states. In the absence of ZFS and when the $2 S+1$ degenerate ground state is well separated from excited energy levels, i.e., the system obeys the Curie law, and Eqs. (125) and (126) can be rewritten as (in SI units)

$$
\begin{aligned}
\boldsymbol{\sigma}^{M} & =\boldsymbol{\sigma}^{M, \text { orb }}-\frac{1}{k T} \frac{S(S+1)}{3} \frac{\mu_{B}}{\hbar \gamma^{M}} \mathbf{g}\left(\mathbf{A}^{M}\right)^{\mathrm{T}}, \\
\mathbf{J}^{M N} & =\mathbf{J}^{M N, \text { orb }}-\frac{1}{k T} \frac{S(S+1)}{3} \frac{1}{h} \mathbf{A}^{M}\left(\mathbf{A}^{N}\right)^{\mathrm{T}},
\end{aligned}
$$

where $k T$ is the thermal energy and $2 S+1$ is the multiplicity of the ground state. Equations (127) and (128) follow closely the theory developed by Kurland and McGravey, ${ }^{146}$ which was originally formulated for the NMR shielding tensor. The inclusion of the ZFS effects and other low-lying excited states would require more involved theoretical descriptions of the $\boldsymbol{\sigma}$ - and J-tensor (for 
more details, see Refs. 147-150). Work in this direction is currently ongoing.

Obtaining the NMR shielding and indirect nuclear spin-spin coupling tensor for paramagnetic species [Eqs. (127) and (128)] requires the calculation of orbital (orb) contributions (described in Sec. VI) and EPR tensors (described in Sec. V). All features described in Secs. V and VI are therefore available for the prediction of pNMR parameters as well. Note that a distinctive feature of the ReSpect program is the calculation of the orbital term using open-shell Kramers-unrestricted KS determinants ${ }^{36,151,152}$ instead of the more commonly used approximation where the orbital term is replaced by the NMR tensor of a closed-shell analog of the system studied. To achieve robust convergence of the response function in the calculation of the orbital term, we use a perturbation-free KS determinant with the magnetization oriented in the direction of the magnetic perturbation.

Summary: The calculation of pNMR parameters by ReSpect is available for doublet and triplet open-shell molecular systems with the degenerate ground state described by a Kramers-unrestricted KS determinant (paramagnetic systems), and it currently includes the following features:

- 4c (DKS) Hamiltonian,

- noncollinear DFT potentials and kernels as listed in Table III,

- resolution-of-the-identity integral acceleration for the Coulomb term (RI-J),

- point and finite models for the nuclear charge,

- point and finite models for the nuclear magnetic dipole moment in nuclear spin-spin couplings,

- PCM solvent model for both perturbation-free ${ }^{45,46}$ and response MOs,

- analysis of spin-orbit and scalar relativistic effects by turning off $\mathrm{SO}$ interactions and by increasing the speed of light (a factor of 10 is usually sufficient to reach the non-relativistic limit),

- MO analysis of the paramagnetic NMR contributions,

- MO analysis of the spin-orbit contributions to the NMR shielding tensor using PT3, ${ }^{118,119}$ and

- hybrid MPI/OpenMP parallelization.

Note that the feature list related to NMR for diamagnetic systems is listed in Sec. VI. Readers can find the up-to-date feature list on http://www.respectprogram.org.

\section{A. pNMR applications}

To demonstrate the performance of the ReSpect program in the prediction of the paramagnetic NMR shifts and indirect nuclear spin-spin couplings, we examined two systems (see Fig. 9), which are predicted to have a triply degenerate $(S=1)$ ground state by scalar relativistic or nonrelativistic theories.

The calculated results of ${ }^{1} \mathrm{H}$ pNMR shifts for a recently synthesized $\left[\operatorname{IrRu}_{2}(\mathrm{dpa})_{4} \mathrm{Cl}_{2}\right]^{+}$complex ${ }^{153}$ are presented graphically in Fig. 10 together with the available experimental data. Whereas the ReSpect program has the unique ability of evaluating ZFS from the LR-TDDFT approach, the ZFS parameter calculated for the present system (less than $40 \mathrm{~cm}^{-1}$, see Sec. X E) was not used in this pNMR study. As demonstrated in Ref. 148, such low ZFS values have only

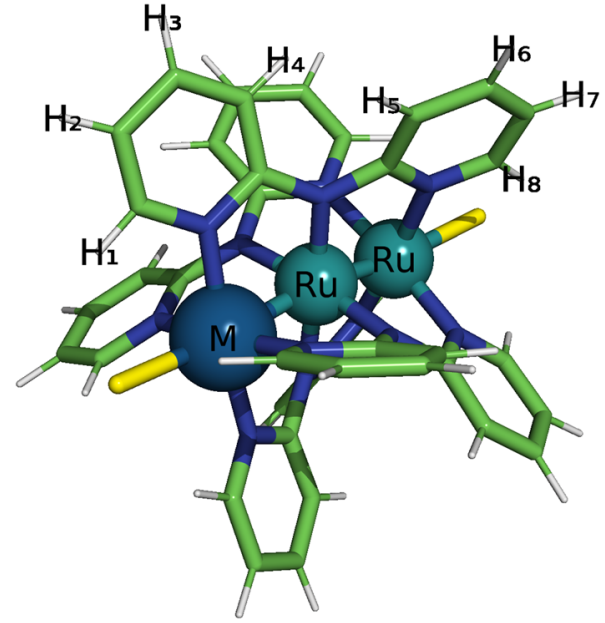

FIG. 9. The molecular structure and atom numbering of $\left[\mathrm{MRu}_{2}(\mathrm{dpa})_{4} \mathrm{Cl}_{2}\right]^{+}$used in the 4c DKS/PBE0 pNMR study in Sec. VII A. M = Rh,Ir.

a minor effect on the pNMR shifts of triplet systems. The calculations were performed at the $4 \mathrm{c}$ DKS/PBE0 ${ }^{80,81,113}$ and DKS/PBE050HFX levels using the geometry obtained from Ref. 153. Dyall's uncontracted valence double-zeta basis ${ }^{82,83,99}$ for $\mathrm{Rh}, \mathrm{Ru}$, and Ir and Jensen's uncontracted pc-1 basis ${ }^{154-157}$ for the light atoms (pcJ-1 for $\mathrm{H})$ were used. The effect of the environment on the NMR parameters was modeled with the IEFPCM solvation model $(\varepsilon=8.93)$.

The effect of different exchange-correlation functionals is minimal for this complex (note the minor difference between the orange and red solid lines). In the original paper, the authors did not specify the reference compound for the ${ }^{1} \mathrm{H}$ shifts or the expression used for calculating the shifts. We therefore converted the calculated

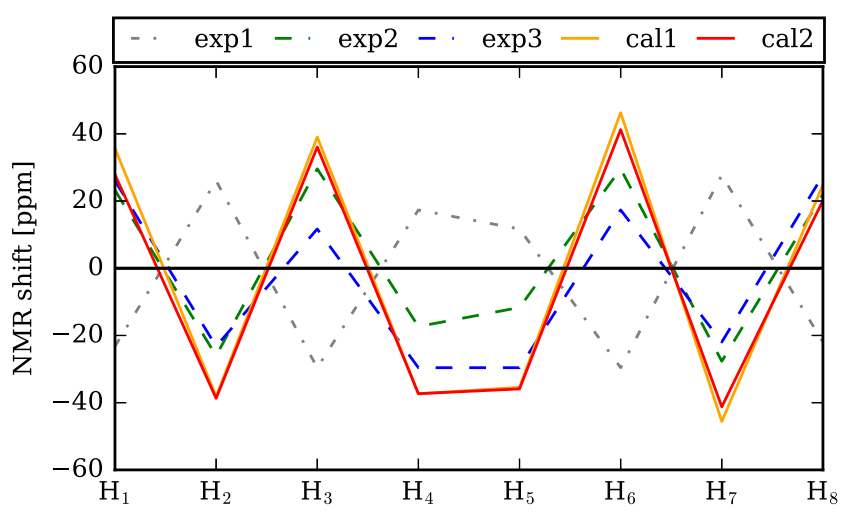

FIG. 10. Experimental (exp) and calculated (cal) data for the complex presented in Fig. 9 with $\mathrm{M}=$ Ir. Here, cal1 and cal2 correspond to the calculations using the PBE0 and PBE0-50HFX functionals, respectively, exp1 refers to the original experimental data taken from Ref. 153, exp2 is obtained by multiplying exp1 by the factor -1 , and exp3 corresponds to the reassignment of exp1 as follows: $\mathrm{H}_{1} \rightarrow \mathrm{H}_{2}, \mathrm{H}_{2} \rightarrow \mathrm{H}_{1}, \mathrm{H}_{3} \rightarrow \mathrm{H}_{5}, \mathrm{H}_{4} \rightarrow \mathrm{H}_{6}, \mathrm{H}_{5} \rightarrow \mathrm{H}_{3}, \mathrm{H}_{6} \rightarrow \mathrm{H}_{4}, \mathrm{H}_{7} \rightarrow \mathrm{H}_{8}$, and $\mathrm{H}_{8} \rightarrow \mathrm{H}_{7}$. 
shieldings to chemical shifts using TMS as the reference compound according to the conventional formula for ${ }^{1} \mathrm{H}$ shifts, $\delta^{M}=\sigma_{\text {ref }}^{M}-\sigma_{\text {calc }}^{M}$. At first glance, the calculated data (presented by solid lines) are in complete disagreement with the original experimental data (the gray dashed-dotted line). However, the agreement becomes much better if one assumes that the authors of the experimental work used an alternative expression for the determination of shifts that results in the change of signs for all ${ }^{1} \mathrm{H}$ peaks (the green dashed line). Alternatively, the agreement can be achieved by assuming that there was misassignment of the signals in the original publication: ${ }^{153}$ the alternative assignment is presented in Fig. 10 as the blue dashed line. It is clear that after reassignment of the proton peaks, the agreement between experimental and calculated results becomes reasonable. Similar conclusions can be drawn for the calculated ${ }^{1} \mathrm{H}$ pNMR shifts for the $\left[\mathrm{RhRu}_{2}(\mathrm{dpa})_{4} \mathrm{Cl}_{2}\right]^{+}$complex presented in Fig. 11. We note that for this complex, the difference between the results obtained with the PBE0 and PBE-50HFX functionals is bigger than for the $\left[\mathrm{IrRu}_{2}(\mathrm{dpa})_{4} \mathrm{Cl}_{2}\right]^{+}$complex. Again, the agreement with experiment becomes much better if one assumes that the authors of the experimental work used an alternative expression for the determination of shifts or accepts the possibility of a misassignment of signals. For the $\left[\mathrm{RhRu}_{2}(\mathrm{dpa})_{4} \mathrm{Cl}_{2}\right]^{+}$complex, we also calculated the ${ }^{3} J_{\mathrm{H}-\mathrm{H}}$ nuclear spin-spin couplings [including the temperature-dependent contribution, see Eq. (126)] as ${ }^{3} J_{1-2}=6.4 \mathrm{~Hz}$ and ${ }^{3} J_{7-8}=5.7 \mathrm{~Hz}$ for PBE0 and ${ }^{3} J_{1-2}=5.4 \mathrm{~Hz}$ and ${ }^{3} J_{7-8}=4.8 \mathrm{~Hz}$ for PBE0-50HFX. These are in good agreement with experiment, ${ }^{3} J_{1-2}=5.7 \mathrm{~Hz}$ and ${ }^{3} J_{7-8}=4.8 \mathrm{~Hz}$. The calculated ${ }^{3} J_{\mathrm{H}-\mathrm{H}}$ provided additional constraints for the possible reassignment of peaks (see Fig. 11 for more details). The remaining deviation between the calculated and experimental shifts in Figs. 10 and 11 can be attributed to the issue of simulating the experimental conditions in the calculations. The counterion present in the experiment was omitted in the calculations, and the calculations were furthermore performed for a static system. Even though we used a polarizable continuum model to simulate the solvent effects, which typically improves agreement with experimental data, this approach is still inferior compared to the inclusion of explicit solvent molecules. On the whole, these examples demonstrate that the ReSpect program has a huge potential for predicting and interpreting the pNMR spectra of compounds containing heavy elements.

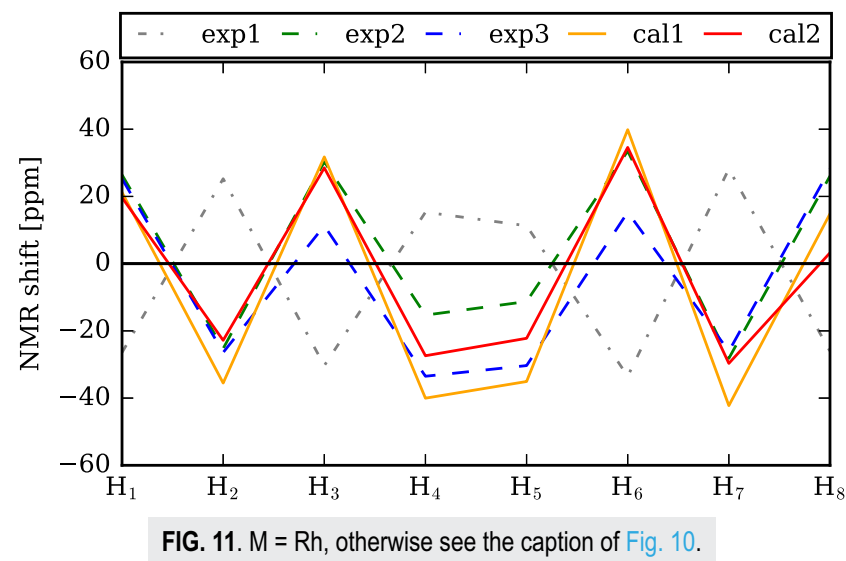

\section{REAL-TIME TDDFT ELECTRON DYNAMICS (RT-TDDFT)}

The methods for dynamical molecular property calculations available in the ReSpect program stem from the solution of the equation of motion for a molecular system subjected to the external electromagnetic field, the Liouville-von Neumann equation (LvN). For Kohn-Sham TDDFT in an orthonormal basis, the LvN equation takes the form

$$
i \frac{\partial}{\partial t} D_{p q}(t)=[\mathbf{F}(t), \mathbf{D}(t)]_{p q}-i \gamma_{p q}\left(D_{p q}(t)-D_{p q}^{\mathrm{eq}}\right)
$$

and describes the time evolution of the one-electron reduced density matrix $\mathbf{D}(t)$. The time evolution is driven by the Fock matrix $\mathbf{F}(t)$ that contains the external electric and/or magnetic fields while taking into account relaxation to the equilibrium (ground) state $\mathbf{D}^{\mathrm{eq}}$ with a rate of relaxation $\gamma_{p q}$ for the $p q$ element of the density matrix.

Real-time (RT) TDDFT represents the most straightforward approach to the solution of the Liouville-von Neumann equation (129): the electronic density is propagated directly in time. ${ }^{158,159}$ Due to this non-perturbative nature, RT-TDDFT allows strong time-dependent external fields of arbitrary shape to be used and non-linear effects in spectra to be described, while at the same time allowing several spectral regions to be addressed in a single run. Moreover, it does not suffer from divergences at resonant frequencies and does not require the evaluation of response kernels. Interested readers can find more discussion on RT-TDDFT in a recent review. ${ }^{160}$

\section{A. RT-TDDFT theory}

The dynamics of electrons in the presence of a time-dependent external field is described by the Liouville-von Neumann equation [Eq. (129)] with the Fock matrix $\mathbf{F}(t)$ expressed in the RKB basis as

$$
\mathbf{F}[\mathbf{D}(t), t]=\mathbf{F}^{0}[\mathbf{D}(t)]+\mathbf{V}^{\mathrm{ext}}(t) .
$$

Here, $\mathbf{F}^{0}[\mathbf{D}(t)]$ is the field-free Fock matrix, which is Hermitian and, due to the presence of the imaginary unit in the Liouville-von Neumann equation, time-reversal general. The Fock matrix $\mathbf{F}^{0}[\mathbf{D}(t)]$ is therefore constructed in the KU formalism, as described in Eq. (77) and in the accompanying discussion. While the Fock matrix in Eq. (130) is assumed to be $4 \mathrm{c}$, it can also be transformed into a $2 \mathrm{c}$ form by means of the $\mathrm{X} 2 \mathrm{C}$ decoupling. ${ }^{40,161}$ In addition, the adiabatic approximation is assumed in the construction of the Fock matrix, i.e., XC potentials developed in the context of time-independent DFT are employed. The second term in Eq. (130), $\mathbf{V}^{\text {ext }}(t)$, is the matrix describing the interaction of the electrons with an external time-dependent field,

$$
\mathbf{V}^{\mathrm{ext}}(t)=-\kappa f(t) \boldsymbol{n} \cdot \mathbf{P},
$$

defined by its amplitude $\kappa$, directional unit vector $\boldsymbol{n}$, a scalar function $f(t)$ defining the field's time dependence, and field-interaction operator P. Currently, ReSpect supports interaction with an external electric field within the dipole approximation, and thus, $\mathbf{P}$ is the matrix representation of the electric dipole moment operator.

The damping parameter $\gamma$ is commonly omitted when solving the LvN equation (129) since it can be applied after the simulation in a post-processing step. The Fock matrix therefore remains 
Hermitian, allowing the use of a unitary propagator. However, due to the implicit time dependence in the Fock matrix, the LvN equation is non-linear and requires the use of sophisticated selfconsistent propagation techniques. ${ }^{162,163}$ Our implementation utilizes the second-order mid-point Magnus propagator ${ }^{164}$ approach, combined with an extrapolation-interpolation scheme, in order to ensure the stability of the time propagation. Details of the solver are described in Ref. 37. At each time step, the density matrix is updated using

$$
\mathbf{D}^{\mathrm{MO}}(t+\Delta t)=\mathbf{U}(t+\Delta t, t) \mathbf{D}^{\mathrm{MO}}(t) \mathbf{U}^{\dagger}(t+\Delta t, t),
$$

with the evolution operator taking the form

$$
\mathbf{U}(t+\Delta t, t) \approx \exp \left[-i \mathbf{F}\left(t+\frac{\Delta t}{2}\right) \cdot \Delta t\right] .
$$

The presence of a future-time Fock matrix $\mathbf{F}\left(t+\frac{\Delta t}{2}\right)$ in Eq. (133) results from the non-linear self-consistent nature of the $\mathrm{LvN}$ equation.

\section{Calculations of spectra}

A straightforward application of RT-TDDFT is the calculation of electronic spectra. Such calculations begin with a timeindependent DFT calculation to obtain the reference density matrix $\mathrm{D}^{\mathrm{MO}}(0)$. This initial state is perturbed by an external field in the form of an initial delta-type pulse, i.e., the function $f(t)$ in Eq. (131) corresponds to the Dirac delta function $\delta\left(t-t_{0}\right)$ centered at the initial time $t_{0}=0$. The ReSpect program analytically applies this pulse and perturbs the initial density matrix $\mathbf{D}^{\mathrm{MO}}(0)$ as

$$
\mathbf{D}^{\mathrm{MO}}\left(0^{+}\right)=e^{i \kappa n \cdot \mathbf{P}} \mathbf{D}^{\mathrm{MO}}(0) e^{-i \kappa n \cdot \mathbf{P}}
$$

where $\kappa, \boldsymbol{n}$, and $\mathbf{P}$ are defined according to Eq. (131). The analytic pulse requires fewer input parameters and allows for a proper description of higher frequencies compared to alternative numerical representations of the delta function, such as the rectangular or Gaussian pulse.

The delta-type pulse excites all electronic modes simultaneously, including both valence and core excited states. However, coreelectron spectra are often hampered by the presence of transitions from valence to high-lying virtual orbitals. Such excitations occur at above-ionization energies and are thus non-physical and represent only an artifact of the finite basis-set representation. One way of identifying these transitions is to recalculate the spectra in various basis sets, as the spurious non-physical peaks are much more sensitive to the changes in the basis set. A much simpler alternative, however, is to keep only certain elements of the perturbation operator, $P_{a i}$, where $i$ corresponds to the targeted core occupied orbitals and $a$ spans sufficiently many virtual orbitals to cover the desired spectral range while setting other elements to zero. ${ }^{41}$ An additional effect of applying this MO window in RT-TDDFT is the possibility of singling out a selected spectroscopic range, as demonstrated in Figs. 12(e) and 12(f).

The perturbed state is then evolved in time, while the induced electric or magnetic dipole moment is recorded on the fly [see Fig. 12(a)]. Depending on the symmetry of the molecule, up to three simulations with external fields in different Cartesian directions may be necessary to capture all of the relevant components of the property tensors. The frequency-dependent molecular properties are obtained by a discrete Fourier transform of the timedomain signal [see Figs. 12(b) and 12(c)] or by means of alternative techniques such as Padé approximants.

Since the damping parameter $\gamma$ is omitted from the Liouvillevon Neumann equation [Eq. (129)], singularities in the absorption spectra arise at resonant frequencies. To regularize these singularities, the time-domain signal is multiplied by the damping factor $e^{-\gamma t}$ during the transformation to the frequency domain, i.e., the Fourier transform is replaced by the Laplace transform. As a result, the absorption spectra consist of Lorentzian-shaped peaks with a finite width.

For a simulation consisting of $N$ time steps of length $\Delta t$, the frequency-domain interval is $(-\pi / \Delta t, \pi / \Delta t)$ with a resolution of $\Delta \omega=2 \pi /(N \Delta t)$. It follows that increasing the resolution of the calculated spectra requires increasing the total simulation length $N \Delta t$. This can be achieved by increasing either the number of time steps (making the simulation more time-consuming) or the size of the time step (putting extra demands on the solver). However, since the frequency-domain interval depends inversely on the time-step length, short time steps are required to describe the high-frequency parts of spectra. Therefore, a balance between the resolution, frequency range, and computational cost has to be made by choosing suitable simulation parameters.

In a weak-field regime, RT-TDDFT yields spectra equivalent to the first order of perturbation theory, while stronger external fields can be used to investigate non-linearities in electronic spectra. However, caution must be exercised with intense fields, since the underlying assumption of the single-determinant nature of the RT-TDDFT electronic state may become invalid. ${ }^{165}$ Higher-order molecular properties can be extracted from time-dependent induced electric or magnetic dipole moments using different post-processing techniques, ${ }^{166}$ aided by the ability of RT-TDDFT to straightforwardly accommodate arbitrary pulse shapes numerically via $\mathbf{V}^{\text {ext }}(t)$ in Eq. (130). As an example, in Ref. 40, an enveloped cosine external field, combined with numerical derivatives with respect to the field strength at each time step, was used to obtain the first and second hyperpolarizabilities in order to investigate relativistic effects on these non-linear optical properties.

\section{Analysis of excitations}

To resolve the longstanding problem of analysis and interpretation of RT-TDDFT signals in terms of MOs, we proposed a dipoleweighted transition matrix analysis. ${ }^{37,41}$ The essence of the method is the Fourier transform of the individual virtual (a)-occupied (i) elements of the induced dipole moment,

$$
\mu_{a i}^{\text {ind }}(t)=P_{i a} D_{a i}(t)+P_{a i} D_{i a}(t) \stackrel{\int d t e^{i \omega t-\gamma t}}{\longrightarrow} \mu_{a i}^{\text {ind }}(\omega),
$$

and the subsequent visualization of $\mu_{a i}^{\text {ind }}(\omega)$ at a selected frequency $\omega$. In addition, this partitioning into virtual-occupied MO contributions leads to an accelerated approach for calculating spectra from RT-TDDFT simulations. The MO-pair contributions $\mu_{a i}^{\text {ind }}(t)$ can be transformed to the frequency domain by means of Padé approximants and then combined into the final spectra. Since the individual frequency-domain quantities $\mu_{a i}^{\text {ind }}(\omega)$ contain much fewer resonant frequencies than the full spectrum, the Padé approximants converge 
Time-dependent electric dipole moment of (g)

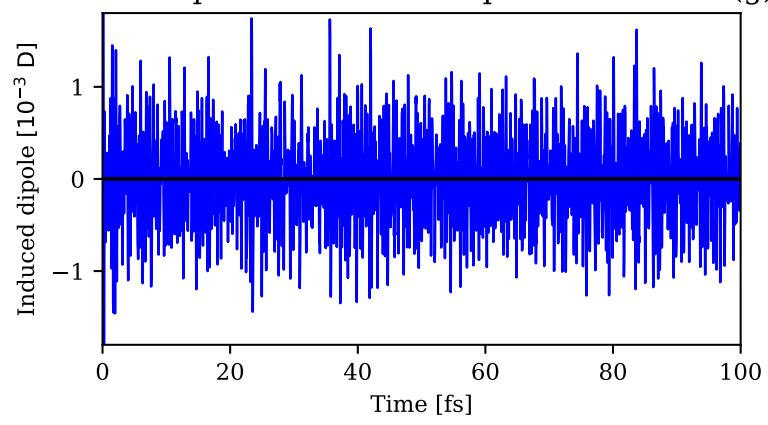

(a)

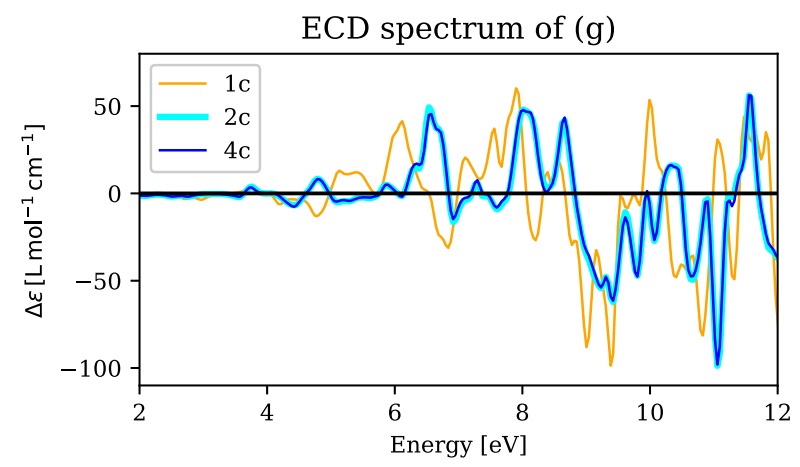

(c)

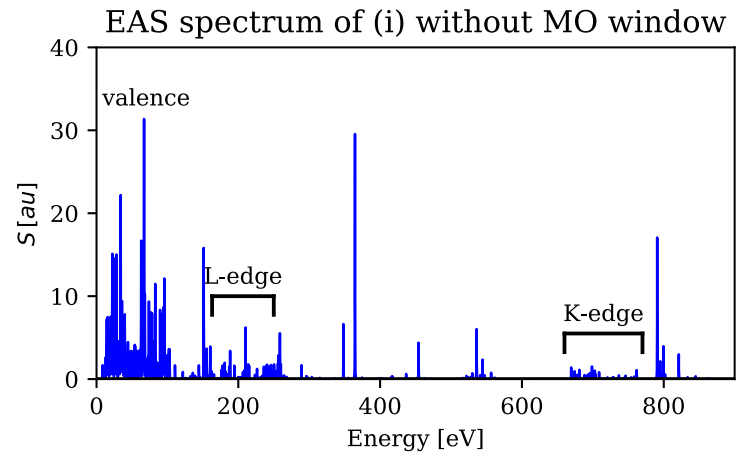

(e)

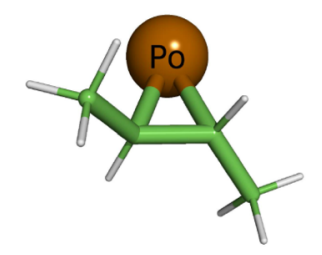

(g)

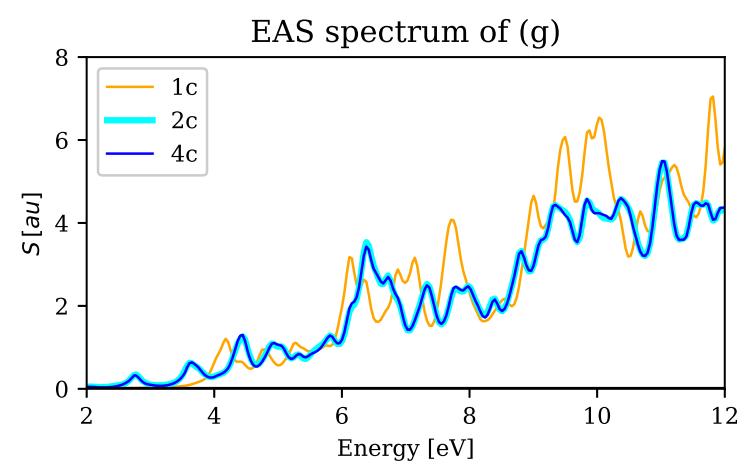

(b)

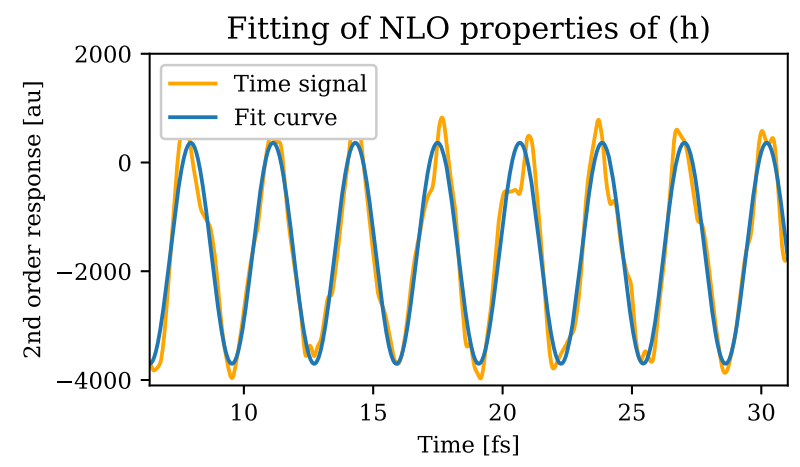

(d)

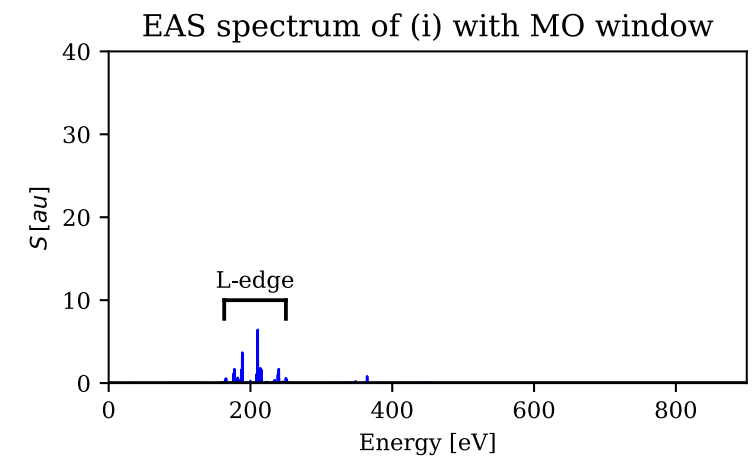

(f)

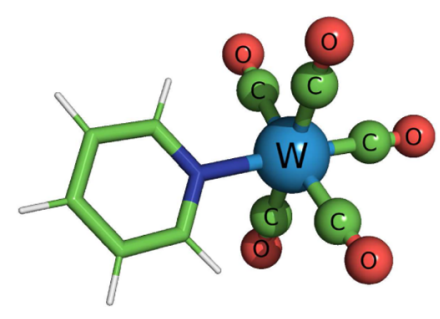

(h)

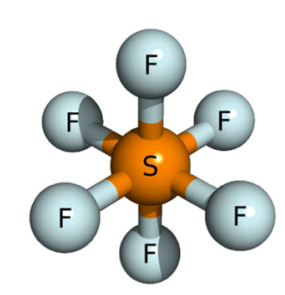

(i)

FIG. 12. Examples of dynamical molecular properties calculated by means of real-time (RT) TDDFT and the molecular systems involved. The properties were treated using both pure and hybrid density functionals and at levels of theory ranging from one-component KS (1c), via quasirelativistic two-component (X2C), to fully relativistic four-component (4c) description. For details and discussion, see Sec. VIII B. (a) Induced time-dependent electric dipole moment, (b) electronic absorption spectrum (EAS), (c) electronic circular dichroism spectrum (ECD), (d) non-linear optical (NLO) properties: the first hyperpolarizabilities, (e) several EAS spectral regions obtained from one simulation, (f) sulfur L-edge spectrum isolated by applying the perturbation to a selected $\mathrm{MO}$ window, (g) $\mathrm{PoC}_{2} \mathrm{H}_{2}\left(\mathrm{CH}_{3}\right)_{2}$, (h) $\left[\mathrm{W}(\mathrm{CO})_{5}(\mathrm{py})\right]$, and (i) $\mathrm{SF}_{6}$. 
and yield spectra with higher resolution from shorter simulations than the Fourier transform. This approach was first advocated by Bruner, LaMaster, and Lopata ${ }^{167}$ and is now available in the ReSpect program package as well.

Summary: The RT-TDDFT electron dynamics module of ReSpect allows the calculation of the following molecular properties: ${ }^{37,40-42}$

- frequency-dependent electric dipole polarizability,

- electronic absorption spectra,

- electronic/natural circular dichroism spectra,

- optical rotatory dispersion spectra

and these are all available for both closed-shell and open-shell molecular systems with the ground state described by Kramersrestricted or Kramers-unrestricted KS determinants. The current list of features includes the following:

- $1 \mathrm{c}(\mathrm{KS}), 2 \mathrm{c}(\mathrm{X} 2 \mathrm{C}),{ }^{40}$ and $4 \mathrm{c}(\mathrm{DKS})^{37}$ Hamiltonians,

- possibility of addressing arbitrary spectral regions (from $\mathrm{UV} /$ vis to $\mathrm{x}$-ray),

- external field of analytic delta, Gaussian, and (linearly enveloped) harmonic form,

- possibility of applying perturbation/response operators only to a selected MO window,

- evaluation of spectra by means of Fourier transform or Padé approximants,

- analysis of spectral lines in terms of MO transitions, ${ }^{37,41}$

- restart from previous calculations,

- resolution-of-the-identity integral acceleration for the Coulomb term (RI-J), ${ }^{42}$ and

- hybrid MPI/OpenMP parallelization.

Readers can find the up-to-date feature list on http://www. respectprogram.org.

\section{B. RT-TDDFT applications}

A collection of RT-TDDFT results for a variety of dynamical molecular properties is shown in Fig. 12. First, the graphs in Figs. 12(a)-12(c) illustrate the process of obtaining electronic spectra. The electric dipole moment induced by a short external electric pulse [Fig. 12(a)] is transformed to the frequency domain, the imaginary part of which corresponds to the electronic absorption spectrum [Fig. 12(b)]. The induced magnetic dipole moment, on the other hand, leads to the electronic circular dichroism spectrum [Fig. 12(c)].

The results depicted in Figs. 12(a)-12(c) are for the dimethylpolonirane molecule, a heavy-element analog of dimethyloxirane [Fig. 12(g)], and a popular test system for chiroptical property calculation methods. The molecule was treated at the $1 \mathrm{c} \mathrm{KS}, 2 \mathrm{c}$ $\mathrm{X} 2 \mathrm{C}$, and $4 \mathrm{c}$ DKS levels of theory using the PBE functional, ${ }^{80,81} \mathrm{com}-$ bined with resolution-of-the-identity for the Coulomb term (RI-J) ${ }^{42}$ and an all-electron uncontracted GTO basis: Dyall's augmented cVDZ basis $^{98}$ for Po, and Dunning's augmented cc-pVDZ basis ${ }^{84}$ for all other elements. In order to calculate the spectra, the molecule was perturbed by a delta-type external field of strength 0.0001 a.u. and left to evolve for 30000 time steps of length 0.15 a.u. (0.0036 fs), which corresponds to the total simulation time of approximately $109 \mathrm{fs}$ and a frequency-domain resolution of 0.0014 a.u. $(0.038 \mathrm{eV})$.
The final spectra were broadened by a damping factor of 0.004 a.u. The aim was to study the importance of relativistic effects in chiroptical spectra with increasing atomic number of the heteroatom. Notably, in the region between $4 \mathrm{eV}$ and $7 \mathrm{eV}$ in Fig. 12(c), the relativistic and non-relativistic electronic circular dichroism (ECD) spectra give the impression of being mirror images, i.e., the use of the non-relativistic method would lead to incorrect enantiomer assignment. Both relativistic Hamiltonians, on the other hand, yield virtually identical results, with the $\mathrm{X} 2 \mathrm{C}$ approach being over 7 times faster than the $4 \mathrm{c}$ calculation. The interested readers can find further results and discussion in Ref. 42 .

Second, Fig. 12(d) illustrates the procedure for obtaining higher-order responses from RT-TDDFT. The second derivative of an induced electric dipole moment with respect to the strength of an external harmonic field reads $\mu_{x x x}^{(2)}(t)=\frac{1}{4}\left[\beta_{x x x}^{\mathrm{SHG}} \cos (2 \omega t)+\beta_{x x x}^{\mathrm{OR}}\right]$, where $\omega$ is the frequency of the external field and the first hyperpolarizabilities $\beta_{x x x}^{\mathrm{SHG}}$ and $\beta_{x x x}^{\mathrm{OR}}$ are the $x x x$ components of the second harmonic generation (SHG) and optical rectification (OR) coefficients, respectively. In order to obtain the second derivative, several simulations with different external field amplitudes are performed and the numerical derivative is taken in each time step. The hyperpolarizability tensors are determined by fitting the simulation results to the analytic formula. The example shown in Fig. 12(d) is that of the $\left[\mathrm{W}(\mathrm{CO})_{5}(\mathrm{py})\right]$ complex, where py stands for pyridine [Fig. 12(h)]. The system was treated at the $\mathrm{X} 2 \mathrm{C}$ level of theory using the B3LYP functional, ${ }^{168-170}$ Dyall's VDZ basis ${ }^{82,83}$ for W and Dunning's augmented cc-pVDZ basis ${ }^{84}$ for all other elements. The system was propagated for 7000 time steps of length 0.15 a.u. under the influence of external fields of cosine time dependence with frequency 0.0239 a.u. and a linear envelope in the first period and amplitudes 0.002 a.u., 0.004 a.u., -0.002 a.u., and -0.004 a.u. Note that shorter overall simulation times were sufficient to calculate the non-linear properties due to the use of the monochromatic harmonic field. It was shown that relativistic effects contributed to about $35 \%$ of the final values of the SHG and OR coefficients. The results were reported in Ref. 40 where further information is available.

Finally, Figs. 12(e) and 12(f) illustrate how a choice of an active $\mathrm{MO}$ window in the perturbation operator in RT-TDDFT allows the selection of one particular spectral range of interest. In the presented electronic absorption spectra of the $\mathrm{SF}_{6}$ molecule [Fig. 12(i)], the application of the full perturbation excited all electronic modes, and as a result, the valence, sulfur L-edge, and carbon K-edge spectra were all obtained together. By restricting the perturbation to sulfur 2 p orbitals only, its L-edge spectrum could be isolated. The calculations were performed using the B3LYP functional, ${ }^{168-170}$ the aug-cc$\mathrm{pV}(\mathrm{T}+\mathrm{d}) \mathrm{Z}$ basis $^{171}$ set for sulfur, and a modified aug-cc-pVTZ basis for fluorine (all $\mathrm{f}$ and the most diffuse $\mathrm{d}$ functions removed). ${ }^{84} \mathrm{An}$ external $\delta$-type pulse with amplitude 0.0005 a.u. was applied to excite the molecule, which was afterward evolved for 56000 time steps of length 0.025 a.u., and the resulting signal was damped with a factor of 0.0038 a.u. Further results, including discussion of the selective perturbation used to remove nonphysical valence-to-continuum excitations in RT-TDDFT simulations, are available in Ref. 41.

\section{DAMPED RESPONSE TDDFT (DR-TDDFT)}

A different approach to solving the Liouville-von Neumann equation, Eq. (129), is the response theory that seeks to obtain 
the density matrix in the form of a perturbation expansion. The resulting method, the so-called damped response (DR) TDDFT [also known in the literature as the complex polarization propagator (CPP) approach], ${ }^{172}$ is a perturbation theory-based method that determines the response of a molecular system directly in the frequency domain for the spectral range of interest while explicitly including the damping parameter $\gamma$ in the Liouville-von Neumann equation, Eq. (129), thus preventing divergences at near-resonant frequencies and allowing the description of absorption processes. The method is therefore well suited for the treatment of highfrequency or high density-of-states spectral regions that may prove challenging for alternative approaches.

The ReSpect program currently contains DR-TDDFT in the linear response regime and allows the calculation of linear electric dipole polarizability, optical rotation, electronic absorption, and electronic circular dichroism spectra in the visible, UV, and $\mathrm{x}$-ray spectral regions. ${ }^{38}$

\section{A. DR-TDDFT theory}

In linear response theory, the calculation of the spectra depends only on the virtual-occupied block of the first-order density matrix, which is parameterized as

$$
D_{a i}^{(1)}(t)=X_{a i} e^{-i \omega t}+Y_{a i}^{*} e^{i \omega t},
$$

where $\omega$ is the frequency of the driving external field and $X_{a i}$ and $Y_{a i}$ are undetermined coefficients. By expanding the Liouville-von Neumann equation, Eq. (129), to first order in the applied perturbation and taking the ansatz in Eq. (136), an algebraic equation

$$
\left[\left(\begin{array}{cc}
\mathbf{A} & \mathbf{B} \\
\mathbf{B}^{*} & \mathbf{A}^{*}
\end{array}\right)-(\omega+i \gamma)\left(\begin{array}{cc}
\mathbf{1} & \mathbf{0} \\
\mathbf{0} & -\mathbf{1}
\end{array}\right)\right]\left(\begin{array}{l}
\mathbf{X} \\
\mathbf{Y}
\end{array}\right)=-\left(\begin{array}{c}
\mathbf{P} \\
\mathbf{P}^{*}
\end{array}\right)
$$

is obtained whose solution is the central problem of DR-TDDFT. The matrices $\mathbf{A}$ and $\mathbf{B}$ are defined in Eq. (115). The right-hand side of Eq. (137) describes the interaction of the molecular system with an external field of frequency $\omega$, and $\gamma$ is a common relaxation (damping) parameter, used as an approximation for the individual relaxation rates $\gamma_{p q}$ of Eq. (129). For now, we only consider interactions with an external electric field within the dipole approximation. In the current release of ReSpect, DR-TDDFT is restricted to molecular systems with closed-shell ground states, and the matrices $\mathbf{A}$ and $\mathbf{B}$ include the noncollinear kernel as described in Eq. (70) and Ref. 38. The adiabatic approximation of TDDFT is assumed. Note that the omission of the damping factor $\gamma$ in Eq. (137) leads to the socalled standard response equation, which, however, cannot describe absorption processes and suffers from divergences at near-resonant frequencies.

The numerical solution of Eq. (137) is performed by means of an iterative subspace algorithm, since, for realistic molecular systems, the size of the matrix on the left-hand side of the equation prohibits its direct inversion or the use of elimination techniques. The essence of such an algorithm is a projection of the large matrix equation onto a basis of trial vectors, yielding a smaller, so-called reduced equation for the expansion coefficients, which can be solved directly. In the iterative subspace solver adapted for relativistic $4 \mathrm{c}$ DR-TDDFT, the response vector is divided into components characterized by their hermicity and time-reversal symmetry that are, however, sought after as linear combinations of trial vectors characterized by their hermicity only. ${ }^{173}$ To achieve stable convergence, it is necessary to use a proper noncollinear kernel, parameterization of trial vectors, and control of numerical precision of algebraic operations. The details of the iterative subspace algorithm implemented in the ReSpect program can be found in Ref. 38.

\section{Calculations of spectra}

The complex linear response functions (susceptibilities) are calculated from the DR-TDDFT response vectors as

$$
\langle\langle R, P\rangle\rangle_{\omega}=X_{a i} R_{i a}+Y_{a i} R_{a i},
$$

where $\mathbf{R}$ is the matrix representation of the operator corresponding to a property induced by the external field. The calculation of spectra with DR-TDDFT then amounts to solving the linear response equation for a number of frequencies in the spectral range of interest in order to obtain the response vectors for each frequency and using Eq. (138) to evaluate the response functions. ReSpect currently supports electric dipole perturbation [right-hand side of Eq. (137)], enabling the calculation of the frequency-dependent linear dipole polarizability, as well as electronic absorption (EAS), optical rotatory dispersion (ORD), and electronic circular dichroism (ECD) spectra. The dispersive and absorptive molecular properties correspond to the real and imaginary parts of the response functions, respectively. Note that in order to get nonzero imaginary parts of the response function, the damping parameter $\gamma$ must have a nonzero positive value.

DR-TDDFT is particularly suited for calculating $\mathrm{x}$-ray spectra, ${ }^{174}$ since it directly calculates molecular responses at the given frequencies. However, when addressing core-excited states with DRTDDFT, non-physical transitions arising from electronic excitations between valence and high-lying virtual orbitals appear, as was the case for RT-TDDFT. A simple prescription for their elimination is to zero out certain elements of the dipole moment operator $P_{a i}$ if orbitals $a$ and $i$ lie outside a selected range corresponding to the spectroscopy of interest. This procedure was introduced in the context of $\mathrm{RT}^{-T_{D D F T}}{ }^{41}$ and is explained in Sec. VIII.

The spectra obtained from damped response theory can be analyzed in terms of transitions between ground-state MOs by considering individual virtual-occupied orbital-pair contributions,

$$
\left[\langle\langle R, P\rangle\rangle_{\omega}\right]_{a i}=X_{a i} R_{i a}+Y_{a i} R_{a i},
$$

i.e., by omitting the summation over repeated indices in Eq. (138). This so-called dipole-weighted transition analysis was first introduced in the context of real-time TDDFT ${ }^{37}$ [see also Eq. (135) and the accompanying discussion] but can be straightforwardly applied in DR-TDDFT. The results of DR-TDDFT transition analysis are identical to the weak-field regime of RT-TDDFT.

Summary: The DR-TDDFT module of ReSpect allows the calculation of the following molecular properties: ${ }^{38}$

- frequency-dependent electric dipole polarizability,

- electronic absorption spectra,

- electronic/natural circular dichroism spectra,

- optical rotatory dispersion spectra

and these are all available for closed-shell molecular systems with a non-degenerate ground state described by a Kramers-restricted 
KS determinant (diamagnetic systems). The current list of features includes the following:

- $1 \mathrm{c}(\mathrm{KS}), 2 \mathrm{c}(\mathrm{X} 2 \mathrm{C})$, and 4c (DKS) Hamiltonians, ${ }^{38}$

- noncollinear DFT potentials and kernels,

- possibility of addressing arbitrary spectral regions (from $\mathrm{UV} /$ vis to $\mathrm{x}$-ray),

- possibility of applying a perturbation only to a selected MO window,

- analysis of spectral lines in terms of MO transitions,

- restart from previous calculations,

- resolution-of-the-identity integral acceleration for the Coulomb term (RI-J), ${ }^{42}$ and

- hybrid MPI/OpenMP parallelization.

Readers can find the up-to-date feature list on http://www. respectprogram.org.

\section{B. DR-TDDFT applications}

\section{Valence spectroscopies}

To illustrate the capabilities of molecular property calculations using DR-TDDFT, we first present an application to the chiral transition metal complex $\Delta$-[Os(phen $\left.)_{3}\right]^{2+}$, where phen stands for phenanthroline [Fig. 13(e), 67 atoms]. This system was the subject of earlier theoretical studies using TDDFT with the approximative scalar ZORA Hamiltonian ${ }^{175-177}$ as well as an experimental investigation. ${ }^{178}$ The results presented here are adapted from our earlier work ${ }^{38}$ and extended by the inclusion of two-component X2C results.

In these calculations, the system was treated at the relativistic four-component (DKS), two-component (X2C), and non-relativistic one-component levels of theory, employing the PBE0 exchangecorrelation functional. ${ }^{80,81,113}$ Uncontracted versions were used of Dyall's cVDZ basis ${ }^{82,83}$ for Os and Dunning's cc-pVDZ ${ }^{84}$ basis for other elements (1545 scalar basis functions, $30902 \mathrm{c}$ and $61804 \mathrm{c}$ basis spinors). The spectral region between $1.5 \mathrm{eV}$ and $5.5 \mathrm{eV}$ was covered in the damped response calculations, with resolution $0.05 \mathrm{eV}$ and 20 points treated simultaneously in the multi-frequency solver regime.

The resulting spectra are presented in Figs. 13(a) and 13(b). The ECD spectra have been multiplied by a factor of 6 in the region between $1.5 \mathrm{eV}$ and $4.0 \mathrm{eV}$ to zoom in on the lower-intensity part of the spectra (the same scaling was applied in Ref. 175). While there is a relatively good agreement in both EAS and ECD spectra between the $1 c, 2 c$, and $4 c$ results, some differences are observed between the results obtained with non-relativistic and relativistic theories. Specifically, the low-frequency peaks (below $4.0 \mathrm{eV}$ ) are red-shifted, and new peaks emerge at the relativistic level of theory. An analysis of the spectrum in terms of MO transitions was applied and revealed that the signals below $4.0 \mathrm{eV}$ result from metal-to-ligand charge transfer (MLCT) excitations from d-type spinors of the central atom to ligand orbitals, while the intense higher-energy transition results from a ligand-to-ligand excitation (corroborating the assignment made in one of the earlier studies ${ }^{175}$ ). The noticeable differences between the relativistic and non-relativistic results in the low-intensity parts of the spectra can thus be explained by their MLCT origin: the orbitals of the central Os atom are expected to be more affected by relativistic effects than orbitals of the lighter ligand atoms.

\section{X-ray spectroscopies}

An interesting example for simulation of $\mathrm{x}$-ray spectra is provided by the neutral complex $\left[\mathrm{RuCl}_{2}(\mathrm{DMSO})_{2}(\mathrm{Im})_{2}\right]$ [Fig. 13(f), 41 atoms], a molecule investigated while developing a protocol for $\mathrm{x}$ ray studies of $\mathrm{Ru}$-based anti-cancer drugs. ${ }^{179} \mathrm{~A}$ specific feature of the complex is the proximity of the $\mathrm{Cl} \mathrm{K}$-edge and $\mathrm{Ru} \mathrm{L} \mathrm{L}_{3}$-edge absorption regions, allowing the accuracy of DFT in the $\mathrm{x}$-ray region to be studied. Moreover, the calculation of $\mathrm{x}$-ray spectra involving $\mathrm{L}$ edges requires a relativistic treatment to correctly describe the $\mathrm{p}_{1 / 2}-\mathrm{p}_{3 / 2}$ splitting, even for elements in the third row of the Periodic Table. $^{41}$

In order to obtain the $\left[\mathrm{RuCl}_{2}(\mathrm{DMSO})_{2}(\mathrm{Im})_{2}\right]$ spectra, linear damped-response calculations were performed at the relativistic four-component (DKS), two-component (X2C), and the non-relativistic one-component levels of theory, employing the PBE0 exchange-correlation functional. ${ }^{80,81,113}$ Uncontracted versions were used of Dyall's cVDZ basis ${ }^{99}$ for Ru and Dunning's cc$\mathrm{pVDZ}^{84,180}$ basis for other elements (893 scalar basis functions, 1786 $2 \mathrm{c}$ and $35724 \mathrm{c}$ basis spinors). The spectral region of $2770-2810 \mathrm{eV}$ was covered with a resolution of $0.1 \mathrm{eV}$, and 100 frequency points were treated simultaneously in the multi-frequency solver. When constructing the perturbation operator, the $\mathrm{Cl} 1 \mathrm{~s}$ and $\mathrm{Ru} 2 \mathrm{p}$ orbitals were selected.

The resulting $\mathrm{x}$-ray spectra are presented in Figs. 13(b) and 13(d). The spectra consist of absorption near the $\mathrm{Cl} \mathrm{K}$-edge as well as at the $\mathrm{Ru} \mathrm{L} \mathrm{L}_{3}$ edge. The calculated spectra were shifted to align the first peak with the value found in the experimental spectrum $(2810 \mathrm{eV})$. The $4 \mathrm{c}$ spectra were shifted by $48 \mathrm{eV}$ and the $2 \mathrm{c}$ and $1 \mathrm{c}$ spectra by an additional $1.06 \mathrm{eV}$ and $10.85 \mathrm{eV}$, respectively.

The MO transition analysis was used to assign peaks to their respective absorption edges. The first peak at $2822 \mathrm{eV}$ corresponds to the transition from $\mathrm{Cl}_{1} \mathrm{~s}_{1 / 2}$ to the LUMO orbital, with the following signals up to $2847 \mathrm{eV}$ also belonging to the $\mathrm{Cl} \mathrm{K}$-edge spectrum. The $\mathrm{Ru} \mathrm{L}_{3}$ edge begins with the intense peak at $2852 \mathrm{eV}$ and $2849.5 \mathrm{eV}$ at the $4 \mathrm{c}$ and $2 \mathrm{c}$ levels, respectively. At the non-relativistic level, the $\mathrm{L}$ absorption edge does not appear in the plotted part of the spectrum but rather starts at $2867 \mathrm{eV}$. Moreover, a 1c methodology without SO coupling predicts only a single L-edge consisting of excitations from all $\mathrm{p}$ orbitals, while both $2 \mathrm{c}$ and $4 \mathrm{c}$ relativistic techniques correctly predict splitting into $\mathrm{L}_{3}$ and $\mathrm{L}_{2}$ edges. This splitting for $\mathrm{Ru}$ is more than $130 \mathrm{eV}$, and, as a result, the $\mathrm{L}_{3}$ and $\mathrm{L}_{2}$ absorption edges are not observed together, highlighting the need for proper treatment of SO interaction when studying $\mathrm{X}$-ray spectra.

A well-known challenge in calculations of molecular spectra in the x-ray region is the shifts of spectral lines with respect to experimental observations. There are several possible causes, including shortcomings of DFT such as the self-interaction error or the absence of proper inclusion of relativistic effects in the case of quasirelativistic or non-relativistic approaches. For example, in the case of $\left[\mathrm{RuCl}_{2}(\mathrm{DMSO})_{2}(\mathrm{Im})_{2}\right]$, one observes (in addition to the absolute shifts) a difference in the energy separation of the $\mathrm{Cl} \mathrm{K}$ edge and $\mathrm{Ru} \mathrm{L} \mathrm{L}_{3}$ edge. In the experiment, this separation is approximately $20 \mathrm{eV}$, while the calculations predict approximately $30 \mathrm{eV}$. This discrepancy is speculated to arise from different DFT self-interaction errors for the two nuclei. Moreover, we can also hypothesize that the difference in the position of the $\mathrm{Ru} \mathrm{L}_{3}$ edge in the $2 \mathrm{c}$ and $4 \mathrm{c}$ calculations arises from the consideration of only the 1-electron part of 


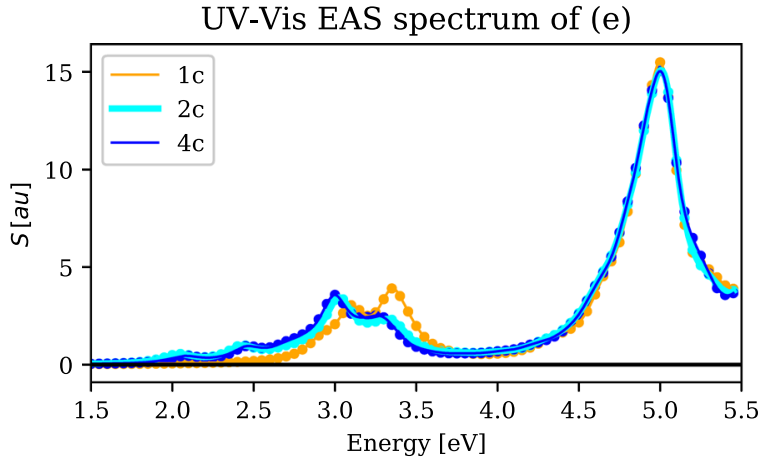

(a)

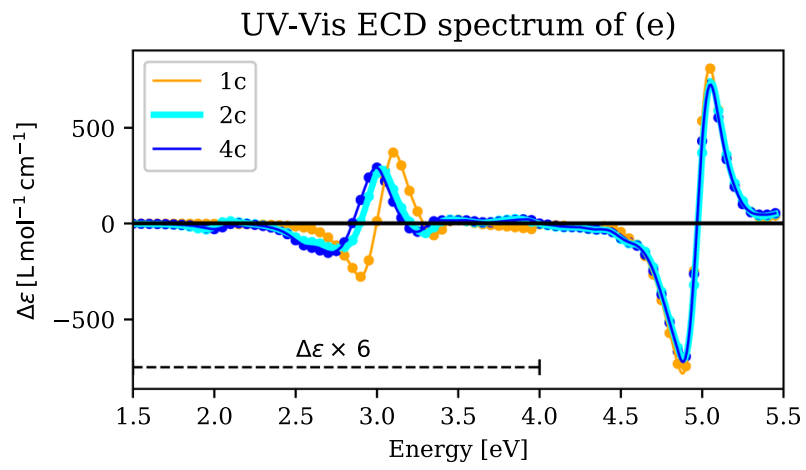

(c)

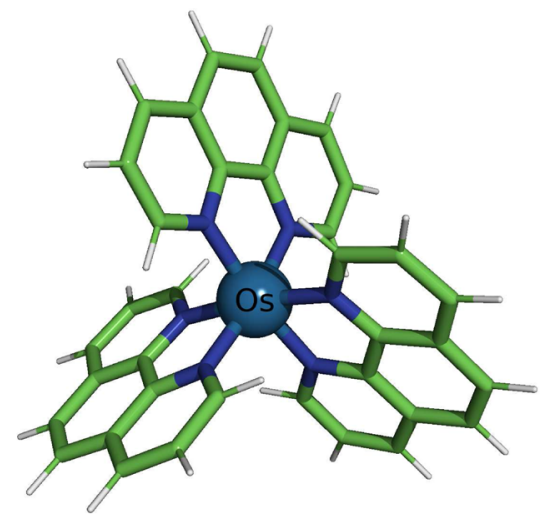

(e)

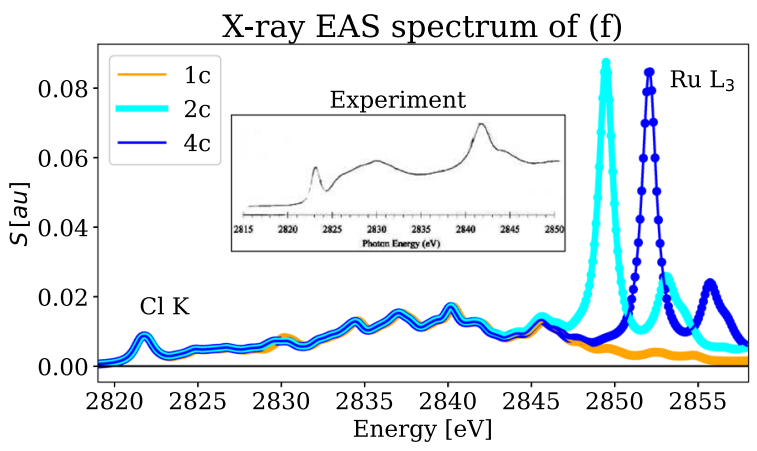

(b)

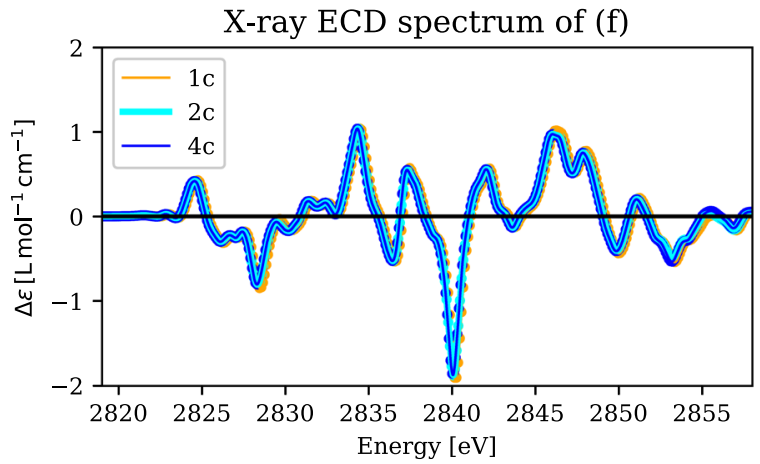

(d)

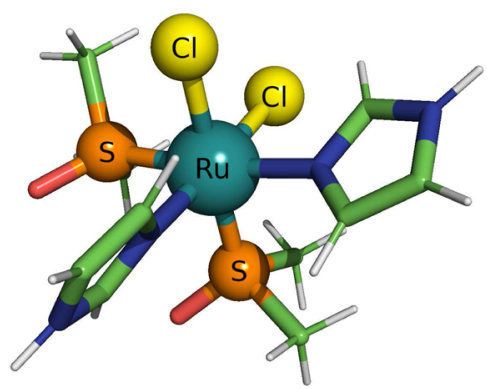

(f)

FIG. 13. Examples of electronic absorption (EAS) and electronic circular dichroism (ECD) spectra in UV-vis and x-ray regions, calculated by means of linear dampedresponse (DR) TDDFT, and the molecular systems involved. The spectra were described at the one-component KS (1c), quasirelativistic two-component X2C (2c), and fully relativistic four-component DKS (4c) levels of theory using the PBE0 hybrid density functional. For discussion and analysis of the spectra, see Sec. IX B. (a) UV-vis EAS, (b) $x$-ray EAS, (c) UV-vis ECD, (d) x-ray ECD, (e) $\Delta$-[Os(phen) $\left.)_{3}\right]^{2+}$, and (f) $\left[\mathrm{RuCl}_{2}(\mathrm{DMSO})_{2}(\mathrm{Im})_{2}\right]$.

the Hamiltonian when generating the $\mathrm{X} 2 \mathrm{C}$ transformation matrix. However, these suggested explanations require further investigation.

In general, though, the calculated spectrum reproduces the essential features of the experimental spectrum, such as the shoulder of the main experimental $\mathrm{Ru} \mathrm{L} \mathrm{L}_{3}$ edge peak, which appears as a separate peak in the calculated spectrum. Moreover, this example serves to illustrate the computational protocol, as well as the methodological advantages and challenges in the application of DR-TDDFT to $\mathrm{x}$-ray spectra. Owing to the formulation of the DR-TDDFT equation, the spectral function can be calculated directly in the frequency domain for the spectral range of interest, without consideration of other parts of the spectrum. In addition, a wide range of frequencies 
can be treated together in the multi-frequency solver. The chemical interpretation of the signals is then aided by the transition analysis.

\section{$X$. EXCITATION ENERGIES FROM LINEAR-RESPONSE TDDFT (LR-TDDFT)}

The central theoretical concept in the calculation of excitation energies in the framework of DFT is that the exact linear response function has poles at the excitation energies of the system. ${ }^{181}$ In response theory, the response of the Kohn-Sham (KS) determinant is induced by changes in the external potential, which are usually caused by electric or magnetic fields. The DFT-based calculation of excitation energies thus corresponds to the calculation of poles of the DFT-based linear response function. The resulting linear-response TDDFT (LR-TDDFT) theory is often referred to as conventional TDDFT or eigenvalue TDDFT theory.

In addition to a simpler approach based on the closedshell Kramers-restricted reference state, we recently have developed a four-component LR-TDDFT approach based on an openshell Kramers-unrestricted reference state (4c-LR-KU-TDDFT). ${ }^{39}$ The implementation in ReSpect is, therefore, applicable to both closed- and open-shell systems and thus capable of addressing a wide variety of relativistic phenomena. Since the method includes spin-orbit effects variationally, it is inherently capable of describing spin-flip and spin-forbidden transitions. In the case of closedshell systems, this leads to nonzero singlet-triplet transition dipole moments, and thus, the method naturally describes the physical phenomenon of phosphorescence. The radiative lifetimes of phosphorescent complexes are of importance for designing efficient organic light-emitting diodes (OLEDs). Furthermore, the 4cLR-KU-TDDFT method treats all states on an equal footing. The first excitation energy of an effective doublet system is therefore zero due to the energetic degeneracy of the Kramers pair. Moreover, fluorescence (singlet-singlet transitions) and phosphorescence (singlet-triplet transitions) are described simultaneously. This also means that the spin-orbit-driven zero-field splitting effect, i.e., the lifting of an otherwise degenerate $2 S+1$ multiplet, can be trivially obtained from the 4c-LR-KU-TDDFT calculations as energetic excitations.

The starting point for LR-TDDFT methods is the Liouvillevon Neumann equation without the damping factor, i.e., without the description of relaxation effects [see Eq. (129) in Sec. VIII]. The poles of the DFT linear response function correspond to the excitation energies and are obtained as solutions of the eigenvalue problem,

$$
\left(\begin{array}{cc}
\mathbf{A} & \mathbf{B} \\
-\mathbf{B}^{*} & -\mathbf{A}^{*}
\end{array}\right)\left(\begin{array}{l}
\mathbf{X}_{N} \\
\mathbf{Y}_{N}
\end{array}\right)=\omega_{N}\left(\begin{array}{l}
\mathbf{X}_{N} \\
\mathbf{Y}_{N}
\end{array}\right)
$$

where $\omega_{N}$ is a vertical electronic excitation energy from the reference electronic state (usually the ground state) to the $N$ th excited electronic state and $\left(\mathbf{X}_{N} \mathbf{Y}_{N}\right)^{\mathrm{T}}$ is the corresponding transition vector. Matrices $\mathbf{A}$ and $\mathbf{B}$ are defined in Eq. (115).

The implemented four-component linear response TDDFT approach is based on a Kramers-unrestricted reference state and utilizes the noncollinear DFT methodology and the modified Davidson-Olsen algorithm (eigenproblem solver). ${ }^{39}$ The former guarantees the invariance of the obtained results with respect to rotations of the coordinate system, and the latter allows one to achieve a robust convergence for systems with both non-degenerate and degenerate ground states.

\section{A. Noncollinear DFT methodology}

The ReSpect program allows the calculation of excitation energies and transition electric dipole moments within the LR-TDDFT method for both closed- and open-shell systems, i.e., for systems with both Kramers-restricted and Kramers-unrestricted KS reference determinants. In Table IV, we summarize all possible combinations of the noncollinear exchange-correlation potential and kernel for closed-and open-shell reference states. It is recommended to use the full GGA expression for both the noncollinear DFT potential and the kernel (the GGA method in Table IV). The use of the ALDA approximation is recommended only for comparison with implementations in other program packages and for its assessment with respect to the full GGA implementation.

\section{B. Eigenvalue solver}

The expression in Eq. (140) has the same matrix form, regardless of the relativistic level of the Hamiltonian. It might therefore seem that the eigenvalue solver (ES) algorithm is independent of the form of the Hamiltonian. This is, however, not the case, and the internal structure of the Hamiltonian greatly influences the form and robustness of different ES algorithms. To obtain good convergence and stability of the ES for systems with degenerate ground states (open-shell Kramers-unrestricted reference states), it is necessary to improve upon the standard Davidson-Olsen algorithm. ${ }^{182-184}$

It was first recognized by Olsen et al. ${ }^{184}$ that one way to stabilize the convergence process of ESs for solving Eq. (140) is to add two (paired) trial vectors in each iteration,

$$
\left(\begin{array}{l}
\mathbf{x} \\
\mathbf{y}
\end{array}\right), \quad\left(\begin{array}{l}
\mathbf{y}^{*} \\
\mathbf{x}^{*}
\end{array}\right) .
$$

The improved version of the Davidson-Olsen algorithm implemented in the ReSpect program considers two extra trial vectors,

TABLE IV. Expressions used by the LR-TDDFT module for the calculation of the noncollinear exchange-correlation potential and kernel. ${ }^{a}$

\begin{tabular}{lcccc}
\hline \hline Reference KS determinant & Method & $E^{\mathrm{xc}}$ & $\boldsymbol{V}^{\mathrm{xc}}$ & $\boldsymbol{K}^{\mathrm{xc}}$ \\
\hline \multirow{2}{*}{ Closed-shell } & GGA $^{\mathrm{b}}$ & $(58)$ & $(67)$ & $(70)$ \\
& ALDA & $(58)$ & $(67)$ & $(72)$ \\
\hline \multirow{2}{*}{ Open-shell } & GGA $^{\mathrm{b}}$ & $(66)$ & $(69)$ & $\mathrm{c}$ \\
& ALDA & $(66)$ & $(69)$ & $\mathrm{d}$ \\
\hline
\end{tabular}

${ }^{\mathrm{a}}$ The numbers in parentheses indicate the corresponding equations in the present article.

${ }^{\mathrm{b}}$ Recommended option.

${ }^{c}$ The noncollinear xc kernel as defined in Ref. 39 by Table I.

${ }^{\mathrm{d}}$ The noncollinear xc kernel as defined by Eq. (25) in Ref. 39, neglecting terms containing the gradients of the charge and spin density. 
which correspond to the left eigenvectors of the eigenproblem (140),

$$
\left(\begin{array}{l}
x \\
y
\end{array}\right), \quad\left(\begin{array}{c}
y^{*} \\
x^{*}
\end{array}\right), \quad\left(\begin{array}{c}
x \\
-y
\end{array}\right), \quad\left(\begin{array}{c}
y^{*} \\
-x^{*}
\end{array}\right)
$$

In our experience, it is usually sufficient to include only the paired trial vectors in Eq. (141) for systems with non-degenerate ground states, i.e., for systems with a single-determinant Kramers-restricted configuration. However, for systems with a degenerate ground state (a Kramers-unrestricted reference KS determinant), the use of all four trial vectors is mandatory. For a more detailed description of ES, see Ref. 39.

\section{Electronic absorption spectroscopy and polarizability from LR-TDDFT}

Transition electric dipole moments between the reference (ground) state and the $N$ th excited state read as

$$
\boldsymbol{t}_{N}=\left(X_{N}\right)_{a i} \boldsymbol{P}_{i a}+\left(Y_{N}\right)_{a i} \boldsymbol{P}_{a i},
$$

where $\boldsymbol{P}_{a i}$ corresponds to the electric dipole moment in the molecular orbital basis,

$$
\boldsymbol{P}_{a i}=\left\langle\varphi_{a}|\boldsymbol{\mu}| \varphi_{i}\right\rangle, \quad \boldsymbol{\mu}=-\left(\boldsymbol{r}-\boldsymbol{R}_{C}\right) .
$$

Here, $\boldsymbol{r}$ is the electron position operator, $\boldsymbol{R}_{C}$ is the nuclear charge center of the molecule, and $\varphi_{i}\left(\varphi_{a}\right)$ are occupied (vacant) fourcomponent molecular orbitals of the reference Slater determinant (see Sec. III). Note that, unlike the analogous Eq. (138) of damped response TDDFT, in Eq. (143), the response vectors do not include any external perturbation, and thus, their contraction with the property matrix yields transition moments rather than directly susceptibilities.

The isotropic part of the complex linear electric dipole polarizability tensor in the framework of the LR-TDDFT can be obtained using the formula

$$
\alpha(\omega)=\frac{1}{3} \sum_{N}\left[\frac{\left|\boldsymbol{t}_{N}\right|^{2}}{\omega+\omega_{N}+i \gamma}-\frac{\left|\boldsymbol{t}_{N}\right|^{2}}{\omega-\omega_{N}+i \gamma}\right],
$$

where the damping factor $\gamma$ describes the rate of relaxation. The electronic absorption spectrum is calculated as the imaginary part of $\alpha(\omega)$,

$$
S(\omega)=\frac{4 \pi \omega}{c} \mathfrak{I}[\alpha(\omega)] .
$$

Similarly, the real part of $\alpha(\omega)$ leads to the frequency-dependent linear electric dipole polarizability.

\section{Radiative lifetime from LR-TDDFT}

The radiative rate $k_{N}$ and radiative lifetime $\tau_{N}$ from the $N$ th electronic state to the ground state can be calculated using the Strickler-Berg relationship ${ }^{185}$ (in SI units),

$$
k_{N}=\frac{1}{\tau_{N}}=\frac{4 \alpha^{3} m_{e} e^{4}}{3 \hbar^{3}\left(4 \pi \varepsilon_{0}\right)^{2}} n \omega_{N}\left|\boldsymbol{t}_{N}\right|^{2},
$$

where $n$ is the refractive index of a medium, $\alpha$ is the fine structure constant, $\omega_{N}$ is the $N$ th excitation energy, and $\boldsymbol{t}_{N}$ represents the transition electric dipole moment.

The experimental radiative lifetime is then obtained as the Boltzmann average over a subset of electronic excitations,

$$
\tau_{\text {bav }}=\frac{1}{k_{\text {bav }}}=\frac{\sum_{N} e^{-\omega_{N} / k T}}{\sum_{N} k_{N} e^{-\omega_{N} / k T}} .
$$

Summary: The LR-TDDFT module of ReSpect allows the calculation of the following molecular properties:

- excitation energies, including zero-field splittings,

- transition electric dipole moments,

- electronic absorption spectra,

- frequency-dependent electric dipole polarizability,

- radiative rates and radiative lifetimes using Boltzmann averaging

and these are all available for closed-shell and open-shell doublet and triplet molecular systems, with the ground state described by Kramers-restricted or Kramers-unrestricted KS determinants. The current list of features includes the following:

- 4c (DKS) Hamiltonian,

- noncollinear DFT potentials and kernels as listed in Table IV,

- solution of the full eigenvalue problem (140) as well as Tamm-Dancoff approximation with $\mathbf{B}=0$,

- restart from previous calculations if these did not finish successfully or, for example, the user requires more excitation energies,

- PCM solvent model,

- resolution-of-the-identity integral acceleration for the Coulomb term (RI-J), ${ }^{42}$ and

- hybrid MPI/OpenMP parallelization.

Readers can find the up-to-date feature list on http://www. respectprogram.org.

\section{E. LR-TDDFT applications}

As our first example of the performance and capabilities of the LR-TDDFT method implemented in ReSpect, we calculated the excited state ZFS and radiative lifetimes of a closed-shell iridium(III) complex. Ir(III) complexes are regarded as the best candidates for the construction of organic light emitting diodes based on phosphorescent emitters. ${ }^{186-188}$ Prediction of the phosphorescence phenomenon is sensitive to the accuracy at which the spin-orbit coupling (SOC) is treated, wherefore the four-component relativistic approach is especially suited for this task. The chosen $\operatorname{Ir}(\mathrm{III})$ complex (see Fig. 14) was studied both experimentally and theoretically in Ref. 189 (labeled therein as complex 1). All calculations were done at the $4 \mathrm{c}$ DKS/PBE0 ${ }^{80,81,113}$ level using the geometry taken from Ref. 189, the uncontracted Dyall's valence triple-zeta basis ${ }^{82,83}$ for Ir, and the uncontracted cc-pVTZ basis ${ }^{84}$ for light atoms. First, we calculated the ZFS of the lowest triplet state, $14.1 \mathrm{meV}$. The value is close to the presented result in Ref. $189,11.6 \mathrm{meV}$, where the SOC effects were included perturbatively. To compute the radiative rate at room temperature, we calculated the first 9 excited states 


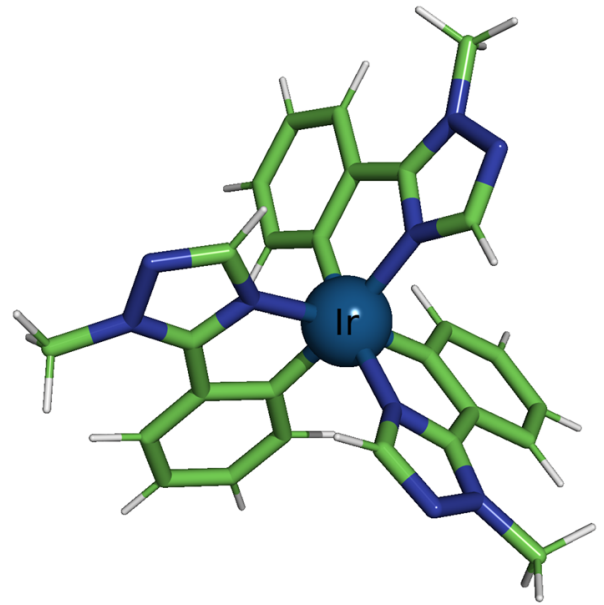

FIG. 14. The molecular structure of $\left[\mathrm{Ir}(\mathrm{ptz})_{3}\right]$ used in the $4 \mathrm{C}$ LR-TDDFT radiative lifetime study (experimental data in Ref. 189), ptz = fac-tris(1-methyl-5-phenyl-3$n$-propyl-[1,2,4]triazolyl).

and used Eqs. (147) and (148). The obtained value $2.2 \times 10^{5} \mathrm{~s}^{-1}$ ( $\tau=4.5 \mu \mathrm{s}$ ) agrees reasonably well with the one presented in Ref. 189, $4.3 \times 10^{5} \mathrm{~s}^{-1}$, although it underestimates the experimental result of $6.1 \pm 0.8 \times 10^{5} \mathrm{~s}^{-1}$. The convergence of the results with the number of excited states was confirmed by calculating 17 states with the double-zeta basis quality. The discrepancy between the calculated and experimental results can be attributed to the lack of environmental effects and to the shortcomings of DFT itself, since as an example, TDDFT favors low-spin states over high-spin states. ${ }^{190,191}$

Finally, we present for the first time the calculation of a groundstate ZFS using the four-component LR-TDDFT methodology. We have applied a recently developed $4 \mathrm{c}-\mathrm{LR}-\mathrm{KU}$-TDDFT method ${ }^{39}$ to the $\left[\mathrm{IrRu}_{2}(\mathrm{dpa})_{4} \mathrm{Cl}_{2}\right]^{+}$complex shown in Fig. 9. The calculations were performed using the $4 \mathrm{c} \mathrm{DKS} / \mathrm{PBE} 0^{80,81,113}$ level of theory with Dyall's uncontracted valence double-zeta basis ${ }^{82,83,99}$ for Ru and Ir and Dunning's uncontracted cc-pVDZ basis ${ }^{84}$ for light atoms. The system has $\mathrm{C}_{1}$ symmetry and has a triply degenerate ground state according to the scalar relativistic or nonrelativistic levels of theory. This degeneracy is lifted in the relativistic theory due to spinorbit coupling. We performed two calculations, with the magnetization oriented along the $z$ and $x$ Cartesian directions with respect to the geometry obtained from Ref. 153 . For the $x$ direction, we obtained a first excitation energy of $22.53 \mathrm{~cm}^{-1}$, and for the $z$ direction, we obtained $-35.46 \mathrm{~cm}^{-1}$. Taking into account that the difference in SCF energies between the $z$ and $x$ directions is $21.41 \mathrm{~cm}^{-1}$, and using the computed first excitation energies, the following ZFS parameters were obtained: $D=24.2 \mathrm{~cm}^{-1}$ and $E=11.3 \mathrm{~cm}^{-1}$. In this work, we have omitted the Gaunt interaction, and therefore, the spin-spin dipole contribution is not included in the calculated values. However, this contribution was found to be insignificant for systems containing heavy elements (see, for example, Ref. 192 and references therein). This implementation of the 4c-LR-KUTDDFT method, the first of its kind, opens the door to new areas of application.

\section{SUMMARY AND OUTLOOK}

Since its inception in 1998 as a program mainly designed to facilitate the exploration of novel ideas and concepts regarding relativistic effects on NMR and EPR parameters, ReSpect has grown into a computationally efficient one-, two-, and four-component relativistic DFT code that can address a wide range of problems in chemistry and physics. The introduction of complex quaternion algebra, restricted kinetic and magnetic balance bases, efficient integral and exchange-correlation routines, and a hybrid MPI/OpenMP parallelization scheme makes it possible to routinely treat molecules with more than 100 atoms at the $4 \mathrm{c}$ relativistic level of theory, and even more at the $2 \mathrm{c} \mathrm{X} 2 \mathrm{C}$ level of theory, even on modest workstations. In recent years, ReSpect has also extended its range of available molecular properties to include real-time, damped response, and linear response TDDFT and now includes both linear and nonlinear frequency-dependent molecular properties involving electric and magnetic fields. At the same time, ReSpect continues to provide unique functionality in the study of relativistic effects on EPR and NMR parameters, including a variety of tools for the analysis and interpretation of the obtained results.

The computational efficiency of the code and the underlying structure used in the implementation of the theory have also allowed ReSpect to include unique features such as Kramersunrestricted Kohn-Sham determinants for systems with a degenerate ground state (paramagnetic systems) as well as efficient handling of Kramers-restricted (closed-shell) systems. The Kramersunrestricted approach in the ReSpect program now offers unique functionality for computational studies of the ground-state zerofield splitting parameters using the LR-TDDFT methodology, the calculation of indirect nuclear spin-spin coupling constants for paramagnetic NMR spectroscopy, and inclusion of solvent effects by means of the polarizable continuum model (also available now for paramagnetic NMR parameters). The first ever applications of these features have been presented in this article. The Kramers-unrestricted approach can also be used to study dynamical (frequency- and time-dependent) properties by means of the linear response and real-time TDDFT modules. A recent extension of the program to the solid-state domain enables fully relativistic four-component all-electron DFT calculations using a Gaussian basis for 1D, 2D, or 3D periodic systems. ${ }^{28}$ This development is expected to allow studies of relativistic phenomena for a wide range of different solid-state materials, particularly for properties depending on the electron density in the vicinity of the nuclei.

ReSpect is still in active development, and we expect new advances in the coming years, such as the possibility of geometry optimizations using external optimization modules in combination with analytic gradients. Nevertheless, ReSpect is designed to be user-friendly, providing a simple input structure and a rich library of basis sets suited for relativistic calculations, a welldocumented manual, and test examples for different calculations. In combination with a variety of different visualization and analysis tools and its computational efficiency, we expect ReSpect to be a valuable part of the toolbox for computational chemists interested in the energetics and properties of heavy element-containing compounds. 


\section{AUTHORS' CONTRIBUTIONS}

All authors contributed equally to this work.

\section{ACKNOWLEDGMENTS}

The authors of ReSpect are indebted to Radovan Bast and Roberto Di Remigio for the development, maintenance, and continuous support of the AutoCMake ${ }^{193}$ and PCMSolver ${ }^{46}$ libraries. The authors are also grateful to Stefan Knecht for his help with pilot MPI parallelization as well as to Jan Vicha and Radek Marek for insightful discussions and for providing some input molecular geometries. This work received support from the Research Council of Norway through a Center of Excellence Grant (Grant No. 262695). Computer time was provided by the Norwegian Supercomputer Program NOTUR (Grant No. NN4654K). Financial support from the Slovak Grant Agencies VEGA and APVV (Contract Nos. 2/0116/17 and APVV-15-0726) is duly acknowledged.

\section{DATA AVAILABILITY}

The data that support the findings of this study are available from the corresponding author upon reasonable request.

\section{REFERENCES}

${ }^{1}$ P. A. M. Dirac, Proc. R. Soc. London, Ser. A 117, 610 (1928).

${ }^{2}$ P. A. M. Dirac, Proc. R. Soc. London, Ser. A 118, 351 (1928).

${ }^{3}$ E. Schrödinger, Phys. Rev. 28, 1049 (1926).

${ }^{4}$ H. Hellmann, J. Chem. Phys. 3, 61 (1935).

${ }^{5}$ M. Dolg, in Handbook of Relativistic Quantum Chemistry, edited by W. Liu (Springer, Berlin, 2017), pp. 449-478.

${ }^{6}$ R. E. Moss, Advanced Molecular Quantum Mechanics (Chapman \& Hall, London, 1973).

${ }^{7}$ M. Reiher and A. Wolf, Relativistic Quantum Chemistry: The Fundamental Theory of Molecular Science (John Wiley \& Sons, 2014).

${ }^{8}$ F. de Groot, Coord. Chem. Rev. 249, 31 (2005).

${ }^{9}$ Y. Ning, G.-Q. Jin, and J.-L. Zhang, Acc. Chem. Res. 52, 2620 (2019).

${ }^{10}$ P. Pyykko, Chem. Rev. 88, 563 (1988).

${ }^{11}$ M. Repisky, S. Komorovsky, R. Bast, and K. Ruud, in Gas Phase NMR, edited by K. Jackowski and M. Jaszuński (The Royal Society of Chemistry, 2016), Chap. 8, pp. 267-303.

${ }^{12}$ J. Vícha, R. Marek, and M. Straka, Inorg. Chem. 55, 1770 (2016).

${ }^{13}$ S. A. Wolf, D. D. Awshalom, R. A. Buhrman, J. M. Daughton, S. von Molnar, M. L. Roukes, A. Y. Chtchelkanova, and D. M. Treger, Science 294, 1488 (2001).

${ }^{14}$ J. E. Moore, Nature 464, 194 (2010).

${ }^{15}$ M. Z. Hasan and C. L. Kane, Rev. Mod. Phys. 82, 3045 (2010).

${ }^{16}$ L. Kou, Y. Ma, Z. Sun, T. Heine, and C. Chen, J. Phys. Chem. Lett. 8, 1905 (2017).

${ }^{17}$ M. Lein, M. Rudolph, S. K. Hashmi, and P. Schwerdtfeger, Organometallics 29, 2206 (2010).

${ }^{18}$ T. B. Demissie, B. D. Garabato, K. Ruud, and P. M. Kozlowski, Angew. Chem., Int. Ed. 55, 11503 (2016).

${ }^{19}$ D. R. Salahub, R. Fournier, P. Mlynarski, I. Papai, A. St-Amant, and J. Ushio, Density Functional Methods in Chemistry (Springer, New York, 1991), pp. 77-100. ${ }^{20}$ V. G. Malkin, O. L. Malkina, and D. R. Salahub, Chem. Phys. Lett. 204, 80 (1993).

${ }^{21}$ L. A. Eriksson, V. G. Malkin, O. L. Malkina, and D. R. Salahub, J. Chem. Phys. 99, 9756 (1993).

${ }^{22}$ V. G. Malkin, O. L. Malkina, and D. R. Salahub, Chem. Phys. Lett. 221, 91 (1994).
${ }^{23}$ V. G. Malkin, O. L. Malkina, M. E. Casida, and D. R. Salahub, J. Am. Chem. Soc. 116, 5898 (1994).

${ }^{24}$ V. G. Malkin, O. L. Malkina, and D. R. Salahub, Chem. Phys. Lett. 261, 335 (1996).

${ }^{25}$ E. van Lenthe, A. van der Avoird, and P. E. S. Wormer, J. Chem. Phys. 108, 4783 (1998).

${ }^{26}$ D. Jayatilaka, J. Chem. Phys. 108, 7587 (1998).

${ }^{27}$ T. Helgaker, P. R. Taylor, K. Ruud, O. Vahtras, and H. Koch, неRмiт, a molecular integral program, 1995.

${ }^{28}$ M. Kadek, M. Repisky, and K. Ruud, Phys. Rev. B 99, 205103 (2019).

${ }^{29}$ M. Repiský, S. Komorovský, E. Malkin, O. L. Malkina, and V. G. Malkin, Chem. Phys. Lett. 488, 94 (2010).

${ }^{30}$ S. Gohr, P. Hrobárik, M. Repiský, S. Komorovský, K. Ruud, and M. Kaupp, J. Phys. Chem. A 119, 12892 (2015).

${ }^{31}$ E. Malkin, M. Repiský, S. Komorovský, P. Mach, O. L. Malkina, and V. G. Malkin, J. Chem. Phys. 134, 044111 (2011).

${ }^{32}$ S. Komorovský, M. Repiský, O. L. Malkina, V. G. Malkin, I. Malkin Ondík, and M. Kaupp, J. Chem. Phys. 128, 104101 (2008).

${ }^{33}$ S. Komorovský, M. Repiský, O. L. Malkina, and V. G. Malkin, J. Chem. Phys, 132, 154101 (2010).

${ }^{34}$ M. Repiský, S. Komorovský, O. L. Malkina, and V. G. Malkin, Chem. Phys. 356, 236 (2009).

${ }^{35}$ S. Komorovsky, M. Repisky, K. Ruud, O. L. Malkina, and V. G. Malkin, J. Phys. Chem. A 117, 14209 (2013).

${ }^{36}$ L. Jeremias, J. Novotný, M. Repisky, S. Komorovsky, and R. Marek, Inorg. Chem. 57, 8748 (2018).

${ }^{37}$ M. Repisky, L. Konecny, M. Kadek, S. Komorovsky, O. L. Malkin, V. G. Malkin, and K. Ruud, J. Chem. Theory Comput. 11, 980 (2015).

${ }^{38}$ L. Konecny, M. Repisky, K. Ruud, and S. Komorovsky, J. Chem. Phys. 151, 194112 (2019).

${ }^{39}$ S. Komorovsky, P. J. Cherry, and M. Repisky, J. Chem. Phys. 151, 184111 (2019).

${ }^{40}$ L. Konecny, M. Kadek, S. Komorovsky, O. L. Malkina, K. Ruud, and M. Repisky, J. Chem. Theory Comput. 12, 5823 (2016).

${ }^{41}$ M. Kadek, L. Konecny, B. Gao, M. Repisky, and K. Ruud, Phys. Chem. Chem. Phys. 17, 22566 (2015).

${ }^{42}$ L. Konecny, M. Kadek, S. Komorovsky, K. Ruud, and M. Repisky, J. Chem. Phys, 149, 204104 (2018).

${ }^{43}$ E. Malkin, S. Komorovsky, M. Repisky, T. B. Demissie, and K. Ruud, J. Phys. Chem. Lett. 4, 459 (2013).

${ }^{44}$ S. Komorovsky, M. Repisky, E. Malkin, T. B. Demissie, and K. Ruud, J. Chem. Theory Comput. 11, 3729 (2015).

${ }^{45}$ R. D. Remigio, M. Repisky, S. Komorovsky, P. Hrobarik, L. Frediani, and K. Ruud, Mol. Phys. 115, 214 (2016).

${ }^{46}$ R. D. Remigio, A. H. Steindal, K. Mozgawa, V. Weijo, H. Cao, and L. Frediani, Int. J. Quantum Chem. 119, e25685 (2018).

${ }^{47}$ I. P. Grant, Relativistic Quantum Theory of Atoms and Molecules: Theory and Computation (Springer Science \& Business Media, 2007).

${ }^{48}$ K. G. Dyall and K. Faegri, Jr., Introduction to Relativistic Quantum Chemistry (Oxford University Press, USA, NY, 2007).

${ }^{49}$ I. P. Grant and H. M. Quiney, Phys. Rev. A 62, 022508 (2000).

${ }^{50}$ L. Visscher and K. G. Dyall, At. Data Nucl. Data Tables 67, 207 (1997).

${ }^{51}$ W. Kutzelnigg and W. Liu, J. Chem. Phys. 123, 241102 (2005).

${ }^{52}$ W. Liu and W. Kutzelnigg, J. Chem. Phys. 126, 114107 (2007).

${ }^{53}$ M. Ilias and T. Saue, J. Chem. Phys. 126, 064102 (2007).

${ }^{54}$ W. Kutzelnigg, Int. J. Quantum Chem. 25, 107 (1984).

${ }^{55}$ W. H. E. Schwarz and H. Wallmeier, Mol. Phys. 46, 1045 (1982).

${ }^{56}$ R. E. Stanton and S. Havriliak, J. Chem. Phys. 81, 1910 (1984).

${ }^{57}$ M. Gell-Mann, Nuovo Cimento 4, 848 (1956).

${ }^{58}$ F. London, J. Phys. Radium 8, 397 (1937).

${ }^{59}$ R. Ditchfield, J. Chem. Phys. 65, 3123 (1976).

${ }^{60}$ N. Rösch, Chem. Phys. 80, 1 (1983).

${ }^{61}$ T. Saue, K. Fægri, T. Helgaker, and O. Gropen, Mol. Phys. 91, 937 (1997).

${ }^{62}$ T. Shiozaki, Mol. Phys. 115, 5 (2016). 
${ }^{63}$ T. Saue and H. J. Aa. Jensen, J. Chem. Phys. 111, 6211 (1999).

${ }^{64}$ K. G. Dyall, J. Chem. Phys. 100, 2118 (1994).

${ }^{65}$ S. Obara and A. Saika, J. Chem. Phys. 84, 3963 (1986).

${ }^{66}$ L. Visscher, Theor. Chem. Acc. 98, 68 (1997).

${ }^{67}$ K. Eichkorn, O. Treutler, H. Öhm, M. Häser, and R. Ahlrichs, Chem. Phys. Lett. 240, 283 (1995).

${ }^{68}$ B. I. Dunlap, J. Mol. Struct.: THEOCHEM 529, 37 (2000).

${ }^{69}$ L. Belpassi, F. Tarantelli, A. Sgamellotti, and H. M. Quiney, J. Chem. Phys. 124, 124104 (2006).

${ }^{70} \mathrm{M}$. Repisky, "Development and implementation of efficient relativistic methods for calculations of NMR and EPR parameters," Ph.D. thesis, Slovak Academy of Sciences, Bratislava, 2009.

${ }^{71}$ M. S. Kelley and T. Shiozaki, J. Chem. Phys. 138, 204113 (2013).

${ }^{72}$ R. D. Reynolds and T. Shiozaki, Phys. Chem. Chem. Phys. 17, 14280 (2015).

${ }^{73}$ C. Van Wüllen, J. Comput. Chem. 23, 779 (2002).

${ }^{74}$ G. Scalmani and M. J. Frisch, J. Chem. Theory Comput. 8, 2193 (2012).

${ }^{75}$ P. Kurz, F. Förster, L. Nordström, G. Bihlmayer, and S. Blügel, Phys. Rev. B 69, 024415 (2004).

${ }^{76}$ K. Knöpfle, L. M. Sandratskii, and J. Kübler, Phys. Rev. B 62, 5564 (2000).

${ }^{77}$ F. G. Eich, S. Pittalis, and G. Vignale, Phys. Rev. B 88, 245102 (2013).

${ }^{78}$ R. Bast, H. J. Aa. Jensen, and T. Saue, Int. J. Quantum Chem. 109, 2091 (2009).

${ }^{79}$ M. Hrdá, T. Kulich, M. Repiský, J. Noga, O. L. Malkina, and V. G. Malkin, J. Comput. Chem. 35, 1725 (2014).

${ }^{80}$ J. P. Perdew, K. Burke, and M. Ernzerhof, Phys. Rev. Lett. 77, 3865 (1996).

${ }^{81}$ J. P. Perdew, K. Burke, and M. Ernzerhof, Phys. Rev. Lett. 78, 1396 (1997).

${ }^{82}$ K. G. Dyall, Theor. Chem. Acc. 112, 403 (2004).

${ }^{83}$ K. G. Dyall and A. S. P. Gomes, Theor. Chem. Acc. 125, 97 (2010).

${ }^{84}$ R. A. Kendall, T. H. Dunning, Jr., and R. J. Harrison, J. Chem. Phys. 96, 6796 (1992).

${ }^{85}$ C. Pisani, Quantum-mechanical Ab-Initio Calculation of the Properties of Crystalline Materials (Springer Science \& Business Media, Berlin, 1996), pp. 33-35.

${ }^{86}$ K. N. Kudin and G. E. Scuseria, Phys. Rev. B 61, 16440 (2000).

${ }^{87}$ R. Łazarski, A. M. Burow, and M. Sierka, J. Chem. Theory Comput. 11, 3029 (2015).

${ }^{88}$ P. E. Blöchl, Phys. Rev. B 50, 17953 (1994).

${ }^{89}$ C. J. Pickard and F. Mauri, Phys. Rev. B 63, 245101 (2001).

${ }^{90}$ L. Maschio, Theor. Chem. Acc. 137, 60 (2018).

${ }^{91}$ M. A. Watson, P. Sałek, P. Macak, and T. Helgaker, J. Chem. Phys. 121, 2915 (2004).

${ }^{92}$ C. L. Berman and L. Greengard, J. Math. Phys. 35, 6036 (1994).

${ }^{93}$ K. N. Kudin and G. E. Scuseria, J. Chem. Phys. 121, 2886 (2004).

${ }^{94}$ M. D. Towler, A. Zupan, and M. Causà, Comput. Phys. Commun. 98, 181 (1996).

${ }^{95}$ G. Dresselhaus, Phys. Rev. 100, 580 (1955).

${ }^{96}$ E. I. Rashba and E. Y. Sherman, Phys. Lett. A 129, 175 (1988).

${ }^{97}$ C.-C. Liu, W. Feng, and Y. Yao, Phys. Rev. Lett. 107, 076802 (2011).

${ }^{98}$ K. G. Dyall, Theor. Chem. Acc. 115, 441 (2006).

${ }^{99}$ K. G. Dyall, Theor. Chem. Acc. 117, 483 (2007).

${ }^{100}$ M. F. Peintinger, D. V. Oliveira, and T. Bredow, J. Comput. Chem. 34, 451 (2013).

${ }^{101}$ N. J. M. Geipel and B. A. Heß, Chem. Phys. Lett. 273, 62 (1997).

${ }^{102}$ R. Zhao, Y. Zhang, Y. Xiao, and W. Liu, J. Chem. Phys. 144, 044105 (2016).

${ }^{103}$ G. T. de Jong and L. Visscher, Theor. Chem. Acc. 107, 304 (2002).

${ }^{104}$ P. J. Cherry, S. Komorovsky, V. G. Malkin, and O. L. Malkina, Mol. Phys. 115, 75 (2017).

${ }^{105}$ R. P. Feynman, Phys. Rev. 56, 340 (1939).

${ }^{106}$ P. Hrobárik, M. Repiský, S. Komorovský, V. Hrobáriková, and M. Kaupp, Theor. Chem. Acc. 129, 715 (2011).

${ }^{107}$ P. L. Bora, J. Novotný, K. Ruud, S. Komorovsky, and R. Marek, J. Chem. Theory Comput. 15, 201 (2019).

${ }^{108}$ P. A. B. Haase, M. Repisky, S. Komorovsky, J. Bendix, and S. P. A. Sauer, Chem. Eur. J. 24, 5124 (2018).
${ }^{109}$ I. Malkin, O. L. Malkina, V. G. Malkin, and M. Kaupp, J. Chem. Phys. 123, 244103 (2005).

${ }^{110}$ M. Kaupp, R. Reviakine, O. L. Malkina, A. Arbuznikov, B. Schimmelpfennig, and V. G. Malkin, J. Comput. Chem. 23, 794 (2002).

${ }^{111}$ M. Munzarová and M. Kaupp, J. Phys. Chem. A 103, 9966 (1999).

${ }^{112}$ C. Remenyi and M. Kaupp, J. Am. Chem. Soc. 127, 11399 (2005).

${ }^{113}$ C. Adamo and V. Barone, J. Chem. Phys. 110, 6158 (1999).

${ }^{114}$ W. Kutzelnigg, U. Fleischer, and M. Schindler, Deuterium and Shift Calculation (Springer Berlin Heidelberg, 1990), pp. 165-262.

${ }^{115}$ M. Beer, J. Kussmann, and C. Ochsenfeld, J. Chem. Phys. 134, 074102 (2011).

${ }^{116}$ H. Larsen, P. Jørgensen, J. Olsen, and T. Helgaker, J. Chem. Phys. 113, 8908 (2000).

${ }^{117}$ T. B. Demissie, J. Chem. Phys. 147, 174301 (2017).

${ }^{118}$ J. Novotný, J. Vícha, P. L. Bora, M. Repisky, M. Straka, S. Komorovsky, and R. Marek, J. Chem. Theory Comput. 13, 3586 (2017).

${ }^{119}$ J. Vícha, S. Komorovsky, M. Repisky, R. Marek, and M. Straka, J. Chem. Theory Comput. 14, 3025 (2018).

${ }^{120}$ P. Hrobarik, V. Hrobarikova, F. Meier, M. Repisky, S. Komorovsky, and M. Kaupp, J. Phys. Chem. A 115, 5654 (2011).

${ }^{121}$ M. Kaupp and O. L. Malkina, J. Chem. Phys. 108, 3648 (1998).

${ }^{122}$ J. Vaara, O. L. Malkina, H. Stoll, V. G. Malkin, and M. Kaupp, J. Chem. Phys. 114, 61 (2001).

${ }^{123}$ J. Vícha, J. Novotný, M. Straka, M. Repisky, K. Ruud, S. Komorovsky, and R. Marek, Phys. Chem. Chem. Phys. 17, 24944 (2015).

${ }^{124}$ A. D. Buckingham and P. J. Stephens, J. Chem. Soc. 1964, 2747.

${ }^{125}$ A. H. Greif, P. Hrobárik, V. Hrobáriková, A. V. Arbuznikov, J. Autschbach, and M. Kaupp, Inorg. Chem. 54, 7199 (2015).

${ }^{126}$ A. H. Greif, P. Hrobárik, and M. Kaupp, Chem. Eur. J. 23, 9790 (2017).

${ }^{127}$ J. Vícha, J. Novotný, S. Komorovsky, M. Straka, M. Kaupp, and R. Marek "Relativistic heavy-neighbor-atom effects on NMR shifts: Concepts and trends across the periodic table," Chem. Rev. (submitted) 2020.

${ }^{128}$ R. J. F. Berger, M. Repisky, and S. Komorovsky, Chem. Commun. 51, 13961 (2015).

${ }^{129}$ P. Hrobárik, V. Hrobáriková, A. H. Greif, and M. Kaupp, Angew. Chem. 51, 10884 (2012).

${ }^{130}$ G. Casella, A. Bagno, S. Komorovsky, M. Repisky, and G. Saielli, Chem. Eur. J. 21, 18834 (2015).

${ }^{131}$ A. C. Castro, H. Fliegl, M. Cascella, T. Helgaker, M. Repisky, S. Komorovsky, M. Á. Medrano, A. G. Quiroga, and M. Swart, Dalton Trans. 48, 8076 (2019).

${ }^{132}$ J. Vícha, R. Marek, and M. Straka, Inorg. Chem. 55, 10302 (2016).

${ }^{133}$ J. Schneider, C. P. Sindlinger, K. Eichele, H. Schubert, and L. Wesemann, J. Am. Chem. Soc. 139, 6542 (2017).

${ }^{134}$ M. Jaszuński, M. Repisky, T. B. Demissie, S. Komorovsky, E. Malkin, K. Ruud, P. Garbacz, K. Jackowski, and W. Makulski, J. Chem. Phys. 139, 234302 (2013).

${ }^{135}$ S. Komorovsky, M. Repisky, E. Malkin, K. Ruud, and J. Gauss, J. Chem. Phys. 142, 091102 (2015).

${ }^{136}$ T. B. Demissie, M. Jaszuński, E. Malkin, S. Komorovský, and K. Ruud, Mol. Phys. 113, 1576 (2015).

${ }^{137}$ T. B. Demissie, M. Jaszuński, S. Komorovsky, M. Repisky, and K. Ruud, J. Chem. Phys. 143, 164311 (2015).

${ }^{138}$ W. H. Flygare, J. Chem. Phys. 41, 793 (1964).

${ }^{139}$ I. A. Aucar, S. S. Gómez, M. C. R. de Azúa, and C. G. Giribet, J. Chem. Phys. 136, 204119 (2012).

${ }^{140}$ Y. Xiao and W. Liu, J. Chem. Phys. 138, 134104 (2013).

${ }^{141}$ G. A. Aucar and I. A. Aucar, Annual Reports on NMR Spectroscopy (Elsevier, 2019), pp. 77-141.

${ }^{142}$ A. Antušek and M. Repisky, Phys. Chem. Chem. Phys. 22, 7065 (2020).

${ }^{143}$ T. B. Demissie, N. Kostenko, S. Komorovsky, M. Repisky, J. Isaksson, A. Bayer, and K. Ruud, J. Phys. Org. Chem. 28, 723 (2015).

${ }^{144}$ A. Kř́stková, S. Komorovsky, M. Repisky, V. G. Malkin, and O. L. Malkina, J. Chem. Phys. 142, 114102 (2015).

${ }^{145}$ M. Jaszuński, A. Antušek, T. B. Demissie, S. Komorovsky, M. Repisky, and K. Ruud, J. Chem. Phys. 145, 244308 (2016). 
${ }^{146}$ R. J. Kurland and B. R. McGarvey, J. Magn. Reson. 2, 286 (1970).

${ }^{147}$ W. Van Den Heuvel and A. Soncini, Phys. Rev. Lett. 109, 073001 (2012).

${ }^{148}$ J. Vaara, S. A. Rouf, and J. Mareš, J. Chem. Theory Comput. 11, 4840 (2015).

${ }^{149}$ B. Martin and J. Autschbach, J. Chem. Phys. 142, 054108 (2015).

${ }^{150}$ P. J. Cherry, S. A. Rouf, and J. Vaara, J. Chem. Theory Comput. 13, 1275 (2017).

${ }^{151}$ J. Novotný, M. Sojka, S. Komorovsky, M. Nečas, and R. Marek, J. Am. Chem. Soc. 138, 8432 (2016).

${ }^{152}$ J. Novotný, D. Přichystal, M. Sojka, S. Komorovsky, M. Nečas, and R. Marek, Inorg. Chem. 57, 641 (2017).

${ }^{153}$ M.-C. Cheng, S.-A. Hua, Q. Lv, M. Sigrist, G.-H. Lee, Y.-C. Liu, M.-H. Chiang, and S.-M. Peng, Dalton Trans. 47, 1422 (2018).

${ }^{154}$ F. Jensen, J. Chem. Phys. 115, 9113 (2001).

${ }^{155}$ F. Jensen, J. Chem. Phys. 116, 3502 (2002).

${ }^{156}$ F. Jensen and T. Helgaker, J. Chem. Phys. 121, 3463 (2004).

${ }^{157}$ F. Jensen, J. Chem. Theory Comput. 2, 1360 (2006).

${ }^{158}$ J. Theilhaber, Phys. Rev. B 46, 12990 (1992).

${ }^{159}$ K. Yabana and G. F. Bertsch, Phys. Rev. B 54, 4484 (1996).

${ }^{160}$ J. J. Goings, P. J. Lestrange, and X. Li, Wiley Interdiscip. Rev. Comput. Mol, Sci. 8(1), e1341 (2018).

${ }^{161}$ J. J. Goings, J. M. Kasper, F. Egidi, S. Sun, and X. Li, J. Chem. Phys. 145, 104107 (2016).

${ }^{162}$ R. Kosloff, J. Phys. Chem. 92, 2087 (1988).

${ }^{163}$ K. Lopata and N. Govind, J. Chem. Theory Comput. 7, 1344 (2011).

${ }^{164}$ W. Magnus, Commun. Pure Appl. Math. 7, 649 (1954).

${ }^{165}$ T. B. Pedersen and S. Kvaal, J. Chem. Phys. 150, 144106 (2019).

${ }^{166}$ F. Ding, B. E. Van Kuiken, B. E. Eichinger, and X. Li, J. Chem. Phys. 138, 064104 (2013).

${ }^{167}$ A. Bruner, D. LaMaster, and K. Lopata, J. Chem. Theory Comput. 12, 3741 (2016).

${ }^{168}$ A. D. Becke, Phys. Rev. A 38, 3098 (1988).

${ }^{169}$ C. Lee, W. Yang, and R. G. Parr, Phys. Rev. B 37, 785 (1988).

${ }^{170}$ P. J. Stephens, F. J. Devlin, C. F. Chabalowski, and M. J. Frisch, J. Phys. Chem. 98, 11623 (1994).

${ }^{171}$ T. H. Dunning, K. A. Peterson, and A. K. Wilson, J. Chem. Phys. 114, 9244 (2001).
${ }^{172}$ P. Norman, D. M. Bishop, H. J. A. Jensen, and J. Oddershede, J. Chem. Phys. 115, 10323 (2001).

${ }^{173}$ S. Villaume, T. Saue, and P. Norman, J. Chem. Phys. 133, 064105 (2010).

${ }^{174}$ P. Norman and A. Dreuw, Chem. Rev. 118, 7208 (2018).

${ }^{175}$ B. Le Guennic, W. Hieringer, A. Görling, and J. Autschbach, J. Phys. Chem. A 109, 4836 (2005).

${ }^{176} \mathrm{M}$. Rudolph and J. Autschbach, J. Phys. Chem. A 115, 2635 (2011).

${ }^{177}$ M. Rudolph and J. Autschbach, J. Phys. Chem. A 115, 14677 (2011).

${ }_{178}$ A. J. McCaffery, S. F. Mason, and B. J. Norman, J. Chem. Soc. A 1969, 1428.

${ }^{179}$ T. V. Harris, R. K. Szilagyi, and K. L. McFarlane Holman, J. Biol. Inorg Chem. 14, 891 (2009).

${ }^{180}$ D. E. Woon and T. H. Dunning, Jr., J. Chem. Phys. 98, 1358 (1993).

${ }^{181}$ P. Norman, K. Ruud, and T. Saue, Principles and Practices of Molecular Properties: Theory, Modeling, and Simulations (Wiley VCH, Chichester, 2018).

${ }^{182}$ E. R. Davidson, J. Comput. Phys. 17, 87 (1975).

${ }^{183}$ J. Olsen, P. Jørgensen, and J. Simons, Chem. Phys. Lett. 169, 463 (1990).

${ }^{184}$ J. Olsen, H. J. A. Jensen, and P. Jørgensen, J. Comput. Phys. 74, 265 (1988).

${ }^{185}$ S. J. Strickler and R. A. Berg, J. Chem. Phys. 37, 814 (1962).

${ }^{186}$ P.-T. Chou and Y. Chi, Chem. Eur. J. 13, 380 (2007).

${ }^{187}$ S.-C. Lo, R. E. Harding, C. P. Shipley, S. G. Stevenson, P. L. Burn, and I. D. W. Samuel, J. Am. Chem. Soc. 131, 16681 (2009).

${ }^{188}$ S. Lamansky, P. Djurovich, D. Murphy, F. Abdel-Razzaq, H.-E. Lee, C. Adachi, P. E. Burrows, S. R. Forrest, and M. E. Thompson, J. Am. Chem. Soc. 123, 4304 (2001).

${ }^{189}$ A. R. G. Smith, M. J. Riley, P. L. Burn, I. R. Gentle, S.-C. Lo, and B. J. Powell, Inorg. Chem. 51, 2821 (2012).

${ }^{190}$ A. Fouqueau, M. E. Casida, L. M. L. Daku, A. Hauser, and F. Neese, J. Chem. Phys. 122, 044110 (2005).

${ }^{191}$ A. Fouqueau, S. Mer, M. E. Casida, L. M. Lawson Daku, A. Hauser, T. Mineva, and F. Neese, J. Chem. Phys. 120, 9473 (2004).

${ }^{192}$ J.-B. Rota, S. Knecht, T. Fleig, D. Ganyushin, T. Saue, F. Neese, and H. Bolvin, J. Chem. Phys. 135, 114106 (2011).

${ }^{193}$ R. Bast, R. Di Remigio, and J. Juselius (2020), “AutoCMake," Zenodo, https://doi.org/10.5281/zenodo.3634941. 